\title{
COLLAPSING IN THE EINSTEIN FLOW
}

\author{
JOHN LOTT
}

\begin{abstract}
We consider expanding vacuum spacetimes with a CMC foliation by compact spacelike hypersurfaces. Under scale invariant a priori geometric bounds (type-III), we show that there are arbitrarily large future time intervals that are modelled by a flat spacetime or a Kasner spacetime. We give related results for a class of expanding vacuum spacetimes that do not satisfy the a priori bounds (type-II).
\end{abstract}

\section{Contents}

1. Introduction 2

1.1. Results 2

1.2. Comparison with Ricci flow $\quad 6$

1.3. Structure of the paper $\quad 7$

1.4. Conventions $\quad 7$

2. Noncollapsed Einstein flows $\quad 7$

2.1. Volume monotonicity 8

2.2. Expanding CMC Einstein flows without a priori bounds 10

2.3. Noncollapsed type-III Einstein flows $\quad 14$

3. Einstein flows on étale groupoids $\quad 20$

3.1. Collapsing limits of expanding CMC Einstein flows $\quad 21$

3.2. Zero dimensional orbit space 24

3.3. One dimensional orbit space $\quad 26$

3.4. Two dimensional orbit space 30

4. Type-II blowdown $\quad 34$

Appendix A. Monotonicity formulas 37

A.1. Curvature formulas under an $\mathbb{R}^{N}$-symmetry 37

A.2. Monotonicity formulas for equivolume foliations $\quad 39$

A.3. Two dimensions $\quad 39$

A.4. Monotonicity of reduced volume $\quad 42$

References

Date: April 17, 2018.

Research partially supported by NSF grant DMS-1510192. 


\section{INTRODUCTION}

This paper is about the future behavior of vacuum Einstein solutions. We make the following assumptions :

(1) We have a globally hyperbolic vacuum spacetime $M$ with a single boundary component which is an initial spacelike hypersurface.

(2) There is a foliation of $M$ by compact $n$-dimensional constant mean curvature (CMC) spacelike hypersurfaces $X$.

(3) The mean curvatures $H$ of the hypersurfaces are future-increasing and range over an interval $\left[H_{0}, 0\right)$, where $H_{0}<0$.

To say a word about the assumptions, there are examples of spatially compact globally hyperbolic vacuum spacetimes without a CMC hypersurface [13. Nevertheless, having a CMC foliation is generally considered to be a fair assumption and it allows one to define a canonical time function, the Hubble time $t=-\frac{n}{H}$. The expanding nature of the spacetime is the statement that $H<0$.

The Lorentzian metric on $M$ can be written as $g=-L^{2} d t^{2}+h(t)$, where $h(t)$ is a Riemannian metric on the compact manifold $X$. It is well known that the vanishing of the Ricci curvature of $g$ can be written as a flow $\mathcal{E}$, parametrized by time $t$, on triples $(h, K, L)$ that satisfy certain constraint equations. Here $K$ is a covariant 2-tensor field on $X$ that becomes the second fundamental form of the time slices. We call $\mathcal{E}$ an Einstein flow.

Fischer and Moncrief found that the normalized spatial volume $(-H)^{n} \operatorname{vol}(X, h(t))$ is monotonically nonincreasing, and constant exactly when $g$ describes a Lorentzian cone over a Riemannian Einstein manifold with Einstein constant $-(n-1)$, i.e. $L=1$ and $h(t)=t^{2} h_{\text {Ein }}$ [16]. (A closely related monotonic quantity was found by Anderson [2].) They suggested that the monotonicity of their normalized volume should imply that for a large part of $X$, in the sense of relative volume, its future development is modelled on a Lorentzian cone of the type mentioned above.

1.1. Results. In this subsection we state the main results of this paper in a somewhat loose form, with references to the precise statements in the body of the paper.

1.1.1. Integral result. We must first introduce the rescaling of an expanding CMC Einstein flow $\mathcal{E}$. Given $s \geq 1$, put $h_{s}(u)=s^{-2} h(s u), K_{s}(u)=s^{-1} K(s u)$ and $L_{s}(u)=L(s u)$. Then $\mathcal{E}_{s}=\left(h_{s}, K_{s}, L_{s}\right)$ is also an expanding CMC Einstein flow. Given $\Lambda>1$, the time interval $\left[\Lambda^{-1}, \Lambda\right]$ for $\mathcal{E}_{s}$ corresponds to the time interval $\left[s \Lambda^{-1}, s \Lambda\right]$ for $\mathcal{E}$. Thus we can analyze the future behavior of $\mathcal{E}$ by understanding the limit as $s \rightarrow \infty$ of $\mathcal{E}_{s}$, on a fixed time interval. It is not hard to see that $\mathcal{E}$ is scale invariant if and only if it describes a Lorentzian cone of the type mentioned above.

There is a pointwise monotonicity statement : $(-H)^{n} \mathrm{~d} \operatorname{vol}(X, h(t))$ is monotonically nonincreasing. Put $\operatorname{dvol}_{\infty}=\lim _{t \rightarrow \infty}(-H)^{n} \operatorname{dvol}(X, h(t))$.

As a consequence of the monotonicity of normalized volume, one obtains an integral result about future evolution. 
Theorem 1.1. (Propositions 2.36 and 2.41) After rescaling, the future evolution becomes increasingly scale invariant, in an integral sense with respect to $\mathrm{dvol}_{\infty}$.

Theorem 1.1 can be considered to say that the Fischer-Moncrief suggestion is true in an integral sense. If $\mathrm{dvol}_{\infty}=0$ then Theorem 1.1 is true but vacuous.

To proceed, we divide the expanding CMC Einstein flows into two types. Using the time vector field, one can make sense of the norm $|\mathrm{Rm}|_{T}$ of the Lorentzian curvature tensor (2.46). Borrowing terminology from Ricci flow, we say that an expanding CMC Einstein flow is type-III if $|\mathrm{Rm}|_{T}=O\left(t^{-2}\right)$, and type-IIb otherwise.

As model spaces, we list the simply connected spatially homogeneous solutions with a future-directed expanding homothetic Killing vector field $\left(\mathcal{L}_{V} g=2 g\right)$ and a spatially compact quotient, in the case $n=3$ [15, p. 187]. They are all type-III.

(1) The Milne spacetime. This is the interior of a forward light cone in the Minkowski space $\mathbb{R}^{1,3}$

(2) The Bianchi-III flat spacetime. This is the product of $\mathbb{R}$ with the interior of a forward light cone in the Minkowski space $\mathbb{R}^{1,2}$.

(3) The Taub-flat spacetime. This is the product of $\mathbb{R}^{2}$ with the interior of a forward light cone in the Minkowski space $\mathbb{R}^{1,1}$.

(4) The Kasner spacetimes on $(0, \infty) \times \mathbb{R}^{3}$, with metric $g=-d u^{2}+u^{2 p_{1}} d x^{2}+u^{2 p_{2}} d y^{2}+$ $u^{2 p_{3}} d z^{2}$. Here $p_{1}+p_{2}+p_{3}=p_{1}^{2}+p_{2}^{2}+p_{3}^{2}=1$.

The Taub-flat spacetime is also the Kasner spacetime with $\left(p_{1}, p_{2}, p_{3}\right)=(1,0,0)$, but we list it separately. Only the Milne spacetime is scale invariant in our earlier sense.

1.1.2. Type-III Einstein flows. In this subsubsection we assume that the Einstein flow $\mathcal{E}$ is type-III. Then we can improve Theorem 1.1 to a pointwise statement.

Theorem 1.2. (Proposition 3.5 and Remark 3.9) Given $x \in X$, if $\operatorname{dvol}_{\infty}(x) \neq 0$ then after rescaling, the future evolution near $x$ becomes increasingly like that of a Lorentzian cone over a Riemannian Einstein space with Einstein constant $-(n-1)$.

The Riemannian Einstein space in the preceding theorem is of a generalized type, as discussed below.

We say that the Einstein flow is noncollapsing if $\mathrm{dvol}_{\infty} \neq 0$. Anderson initiated the study of noncollapsing type-III Einstein flows using rescaling, monotonicity and compactness results [2]. We recapitulate these results in Subsection 2.3. Most of this paper is concerned with the collapsing case, i.e. when $\mathrm{dvol}_{\infty}$ vanishes. The main point of the paper is to make use of results on Einstein flows with continuous spatial symmetries. Einstein flows with symmetries have long been studied in general relativity as toy models. As in [22], our viewpoint is rather that information about Einstein flows with symmetries can give information about all Einstein flows that satisfy an a priori curvature bound.

The appearance of continuous symmetries in collapsing Riemannian manifolds, under uniform sectional curvature bounds, is known from work of Margulis, Gromov, Cheeger, Fukaya and many others. In this paper we promote this to type-III Einstein flows, in analogy to earlier work on type-III Ricci flows [22]. To describe the idea, consider first a 
manifold $X$ with a sequence of Riemannian metrics that collapse with uniformly bounded curvature. To analyze the geometry near a point $x \in X$, one approach is to pass to finite covers of $X$, if possible, that have a noncollapsed pointed limit. This unwrapping approach was used for the Einstein flow by Anderson in [2]. Another approach is to pull back metrics to a ball in $T_{x} X$, using the exponential map, and pass to a noncollapsed pointed limit. Both of these methods work well for local regularity issues in the Einstein flow. However, to obtain nonlocal results, for example to apply monotonicity formulas, it is necessary to have a global approach. For example, in the tangent space approach, it is necessary to glue together the various noncollapsed limits on the balls in the tangent spaces $T_{x} X$, with their local symmetries, as one varies $x$. A convenient language to do this is that of étale groupoids, as used for the Ricci flow in [21] and [22]. A collapsing sequence of pointed $n$-dimensional Riemannian manifolds, with uniformly bounded curvature, has a subsequential limit that is a pointed $n$-dimensional Riemannian groupoid. The Riemannian groupoid is an object with local symmetries; its orbit space is the Gromov-Hausdorff limit of the collapsing Riemannian manifolds, and it also retains information about the limit of their universal covers.

Theorem 1.3. (Corollary 2.54) Given a type-III Einstein flow $\mathcal{E}$ on a pointed $n$-dimensional manifold, if $\left\{t_{i}\right\}_{i=1}^{\infty}$ is a sequence tending to infinity then after passing to a subsequence, the rescalings $\mathcal{E}_{t_{i}}$ converge to a type-III Einstein flow $\mathcal{E}^{\infty}$ on a pointed $n$-dimensional étale groupoid.

The convergence in Theorem 1.3 is in the weak $W^{2, p}$-topology for any $p<\infty$, and in the $C^{1, \alpha}$-topology for any $\alpha \in(0,1)$.

In the rest of this subsubsection, we assume that $n=3$ and $X$ is aspherical, i.e. has contractible universal cover. Then the limit Einstein flow $\mathcal{E}^{\infty}$ is of the type that occurs in dimensional reduction. It lives on an orbifold $X^{\infty}$, which is the orbit space of $\mathcal{E}^{\infty}$. When $X^{\infty}$ is not a point, the fields on $X^{\infty}$ consist of a quintuple $\left(h^{\infty}, K^{\infty}, L^{\infty}, G^{\infty}, A^{\infty}\right)$ where $h^{\infty}$ is a Riemannian metric, $K^{\infty}$ is a covariant 2-tensor field, $L^{\infty}$ is a function, $G^{\infty}$ is locally an $N \times N$ positive definite matrix and $A^{\infty}$ is locally an $\mathbb{R}^{N}$-valued 1 -form. Here $N=3-\operatorname{dim}\left(X^{\infty}\right)$.

Thus we are reduced to understanding the future behavior of $\mathcal{E}^{\infty}$. To do so, we again use monotonic quantities. We need to assume that there is some $D<\infty$ so that the original flow $\mathcal{E}$ has $\operatorname{diam}(X, h(t)) \leq D t$. This ensures that $X^{\infty}$ is compact.

We now list the results about type-III Einstein flows in order of increasing dimension of $X^{\infty}$. All of the results have consequences for the pointed future behavior of the lift of the Einstein flow $\mathcal{E}$ to the universal cover $\widetilde{X}$, that do not invoke groupoids (Corollaries 3.16, 3.33 and 3.53 . We say that an Einstein flow on an étale groupoid is of Kasner type if it is locally isometric to a Kasner solution, and similarly for the other model solutions. We first consider the case when the orbit space $X^{\infty}$ is a point.

Theorem 1.4. (Corollary 3.15) Suppose that the original Einstein flow $\mathcal{E}$ is such that $\lim \inf _{t \rightarrow \infty} t^{-1} \operatorname{diam}(X, h(t))=0$. Then there is a sequence $\left\{t_{i}\right\}_{i=1}^{\infty}$ going to infinity such that the rescaled solutions $\mathcal{E}_{t_{i}}$ approach an Einstein flow of Kasner type. 
We now assume that we are not in the situation covered by Theorem 1.4, and consider the case when the orbit space $X^{\infty}$ is one dimensional. To analyze the future behavior of the limit flow $\mathcal{E}^{\infty}$, we use monotonic quantities from Appendix A. To do so, we need to make an assumption about the existence of an equiareal foliation.

Theorem 1.5. (Proposition 3.29) Suppose that any limit Einstein flow has an orbit space of positive dimension, and there is a limit Einstein flow $\mathcal{E}^{\infty}$ whose orbit space is one dimensional. Suppose that there is a time function $\widehat{u}$ for the limit flow $\mathcal{E}^{\infty}$ that is comparable to the time function $u$ for $\mathcal{E}^{\infty}$, with the property that $\operatorname{det}(G)$ is constant on level sets of $\widehat{u}$ (Assumption 3.26). Then there is a sequence $\left\{t_{i}\right\}_{i=1}^{\infty}$ going to infinity such that the rescaled solutions $\mathcal{E}_{t_{i}}$ approach an Einstein flow of Taub-flat type.

Next, we assume that we are not in the situations covered by Theorems 1.4 and 1.5 , and consider the case when the orbit space $X^{\infty}$ is two dimensional. To analyze the future behavior of the limit flow $\mathcal{E}^{\infty}$, we again use monotonic quantities from Appendix $\mathrm{A}$. We now need to make an assumption about the existence of a CMC foliation on a conformally related three-dimensional Lorentzian metric.

Theorem 1.6. (Proposition 3.49) Suppose that any limit Einstein flow has an orbit space of dimension at least two, and there is a limit Einstein flow $\mathcal{E}^{\infty}$ whose orbit space is two dimensional. Suppose that there is a time function $\widehat{u}$ for the limit flow $\mathcal{E}^{\infty}$ that is comparable to the time function $u$ for $\mathcal{E}^{\infty}$, so that the level sets of $\widehat{u}$ have constant mean curvature for the conformally modified Lorentzian metric $\widehat{g}$ of (3.37) (Assumption 3.46). Then there is a sequence $\left\{t_{i}\right\}_{i=1}^{\infty}$ going to infinity such that the rescaled solutions $\mathcal{E}_{t_{i}}$ approach an Einstein flow of Bianchi-III flat type.

Finally, if a limit flow has an orbit space of dimension three then the rescalings of $\mathcal{E}$ approach a spatially compact quotient of the Milne spacetime (Proposition 2.56).

We made some additional assumptions in Theorems 1.5 and 1.6. There is some flexibility in the precise assumptions to make. Under weaker assumptions, one can prove integral convergence results (Propositions 3.23, 3.41 and 3.44). We need some assumptions to apply the monotonicity results of Appendix $\mathrm{A}$, which are an ingredient in our description of the future behavior of $\mathcal{E}^{\infty}$. Any other way to describe the future behavior would also work.

1.1.3. Type-II Einstein flows. The type-III condition is generally not stable under perturbation [25, 26]. Hence it is relevant to obtain information about expanding CMC Einstein flows that are not type-III. Following Ricci flow terminology, we call them type-IIb Einstein flows. Given such an Einstein flow $\mathcal{E}$ and a time $\widehat{t}$, let $x_{\widehat{t}}$ be a point on the time- $\widehat{t}$ slice where $|\mathrm{Rm}|_{T}$ is maximized. One can rescale the Einstein flow by $|\operatorname{Rm}|_{T}\left(x_{\hat{t}}, \widehat{t}\right)$ and shift the time parameter so that the new flow has $|\mathrm{Rm}|_{T}$ maximized by one on the time-0 slice. With an appropriate choice of parameters $\left\{\widehat{t}_{i}\right\}_{i=1}^{\infty}$ tending to infinity, these pointed rescaled flows converge to an Einstein flow $\mathcal{E}^{\infty}$. It exists for all times $u \in \mathbb{R}$, possibly on an étale groupoid. 
Theorem 1.7. (Corollary 4.6) When $n=3$, if the type-IIb Einstein flow $\mathcal{E}$ has its second fundamental form $K$ controlled by the mean curvature $H$, then $\mathcal{E}^{\infty}$ is a static flat Einstein flow.

Theorem [1.7 applies to the locally homogeneous examples in [25]. The theorem may sound paradoxical, because the rescaled flows have $|\mathrm{Rm}|_{T}$ equal to one at their basepoints, whereas the limit flow is flat. The point is that the metrics converge in the weak $W^{2, p_{-}}$ topology. This is not enough to give pointwise convergence of the curvature norm, even in the locally homogeneous case. The interpretation is that the type-IIb Einstein solution has increasing fluctuations of the curvature tensor, at least near points of maximal curvature, that average it out to zero; c.f. Corollary 4.7. We do however have convergence to the flat metric in the $C^{1, \alpha}$-topology for any $\alpha \in(0,1)$.

1.2. Comparison with Ricci flow. One can compare expanding CMC Einstein flows, on compact three dimensional manifolds, to immortal Ricci flows on compact three dimensional manifolds. (A Ricci flow is immortal if it exists for $t \in[0, \infty)$.) There are some common features.

(1) There is a natural rescaling, and hence notions of type-III and type-IIb solutions.

(2) There is a notion of a self-similar solution. For Ricci flow, this is a Ricci soliton. For Einstein flow, this is a Lorentzian metric with a timelike homothetic vector field.

(3) There is a classification of homogeneous self-similar solutions for the contractible Thurston geometries. The geometries $\mathbb{R}^{3}, H^{3}, H^{2} \times \mathbb{R}, N i l$ and Sol admit selfsimilar Ricci flow solutions. The geometries $\mathbb{R}^{3}, H^{3}$ and $H^{2} \times \mathbb{R}$ admit self-similar Einstein flow solutions.

(4) The normalized volume form is nonincreasing.

(5) Type-III Ricci flows with a scale invariant a priori diameter bound become increasingly homogeneous [22]. The same is true for type-III Einstein flows with a scale invariant a priori diameter bound, at least to the extent proven in this paper.

On the other hand, there are important differences.

(1) As a weakly parabolic flow, the Ricci flow is smoothing (in the right coordinates), as seen by Shi's local derivative estimates. In particular, this allows one to take smooth limits. On the other hand, when taking limits of Einstein flows, one cannot expect the limits to be much better than $W^{2, p}$-regular.

(2) An immortal three dimensional Ricci flow is always type-III [4]. Expanding CMC Einstein flows need not be type-III.

(3) Given a Thurston type, if there is a homogeneous expanding Ricci soliton with that geometry then it is unique. The analogous statement is not true for Einstein flows, as the Kasner solutions all have Thurston type $\mathbb{R}^{3}$.

(4) Considering immortal homogeneous Ricci flows, there is a single transmutation: under the Ricci flow, a homogeneous $\widetilde{\mathrm{SL}(2, \mathbb{R})}$ geometry has a rescaling limit with $H^{2} \times \mathbb{R}$ geometry [21]. On the other hand, there are three transmutations for typeIII homogeneous Einstein flows: a homogeneous $\widetilde{\mathrm{SL}(2, \mathbb{R})}$ geometry has a rescaling 
limit with $H^{2} \times \mathbb{R}$ geometry, and a homogeneous $N i l$ or Sol geometry has a rescaling limit with $\mathbb{R}^{3}$ geometry.

On a technical level, in [22] we showed that any type-III Ricci flow, with a scale invariant a priori diameter bound, becomes increasingly homogeneous as time increases. In the present paper we only show that there are large future time intervals on which the Einstein flow becomes increasingly homogeneous. The reason for the stronger conclusion in [22] is that we had unconditional results for the long-time behavior of the limit Ricci flows, and hence could apply contradiction arguments to get uniform statements about the long-time behavior of the original Ricci flow. In the present paper, Assumptions 3.26] and 3.46 are needed in order to characterize the future behavior of the limit Einstein flows. Because of this, we cannot apply contradiction arguments to get uniform statements about the future behavior of the original Einstein flow.

1.3. Structure of the paper. Section 2 is about noncollapsed expanding CMC Einstein flows, first without any a priori curvature assumptions and then with a type-III curvature assumption. Section 3 concerns collapsing type-III Einstein flows. Section 4 is about type-IIb Einstein flows. More detailed descriptions are at the beginnings of the sections.

Appendix A has monotonicity formulas for expanding CMC $n$-dimensional Einstein flows with a local $\mathbb{R}^{N}$-symmetry. When $n=3$, the monotonic quantities largely reduce to those considered in [5], [10] and [11]. We work in the more general setting partly because, in our opinion, the derivations become clearer and simpler there.

I thank Mike Anderson and Jim Isenberg for helpful discussions. I also thank Mike for comments on an earlier version of this paper.

1.4. Conventions. Convergence in $W^{k, p}$ will mean convergence for all $p<\infty$. Convergence in $C^{k, \alpha}$ will mean convergence for all $\alpha \in(0,1)$. We will use the Einstein summation convention freely.

\section{Noncollapsed Einstein FlOWS}

In this section we give results about Einstein flows with a scale invariant lower volume bound. Subsection 2.1 gives the definitions of Einstein flow, CMC Einstein flow and expanding CMC Einstein flow. We then recall the monotonicity of normalized volume from [16].

In Subsection 2.2 we consider expanding CMC Einstein flows with compact spacelike hypersurfaces, but no a priori curvature bounds. We show that in an integral sense, relative to the limiting normalized volume form, for large time the rescaled flow is asymptotically scale invariant.

Subsection 2.3 is about long-time results for noncollapsed type-III expanding CMC Einstein flows, due largely to Anderson [2]. We give relevant notions of convergence of a sequence of Einstein flows. We define the type-III condition and show that with a lower volume bound and an upper diameter bound, one gets convergence (after rescaling) to the space of Lorentzian cones over Riemannian Einstein manifolds with Einstein constant 
$-(n-1)$. The rest of the subsection is devoted to what one can say without the upper diameter bound.

More detailed descriptions are at the beginnings of the subsections.

\subsection{Volume monotonicity.}

Definition 2.1. Let $I$ be an interval in $\mathbb{R}$. An Einstein flow $\mathcal{E}$ on an $n$-dimensional manifold $X$ is given by a family of nonnegative functions $\{L(t)\}_{t \in I}$ on $X$, a family of Riemannian metrics $\{h(t)\}_{t \in I}$ on $X$, and a family of symmetric covariant 2-tensor fields $\{K(t)\}_{t \in I}$ on $X$, so that if $H=h^{i j} K_{i j}$ and $K^{0}=K-\frac{H}{n} h$ then the constraint equations

$$
R-\left|K^{0}\right|^{2}+\left(1-\frac{1}{n}\right) H^{2}=0
$$

and

$$
\nabla_{i} K^{i}{ }_{j}-\nabla_{j} H=0
$$

are satisfied, along with the evolution equations

$$
\frac{\partial h_{i j}}{\partial t}=-2 L K_{i j}
$$

and

$$
\frac{\partial K_{i j}}{\partial t}=L H K_{i j}-2 L h^{k l} K_{i k} K_{l j}-L_{; i j}+L R_{i j} .
$$

For now, we will assume that $X$ is compact and connected, and that all of the data is smooth. At the moment, $L$ is unconstrained; it will be determined by the elliptic equation (2.13) below. We will generally want $L(t)$ to be positive.

An Einstein flow gives rise to a Ricci-flat Lorentzian metric

$$
g=-L^{2} d t^{2}+h(t)
$$

on $I \times X$, for which the second fundamental form of the time- $t$ slice is $K(t)$. Conversely, given a Lorentzian metric $g$ on a manifold with a proper time function $t$, we can write it in the form (2.6) by using the flow of $\frac{\nabla t}{|\nabla t|^{2}}$ to identify nearby leaves. Letting $K(t)$ be the second fundamental form of the time- $t$ slice, the metric $g$ is Ricci-flat if and only if $(L, h, K)$ is an Einstein flow.

Definition 2.7. A CMC Einstein flow is an Einstein flow for which $H$ only depends on t. It is expanding if $I=\left[t_{0}, \infty\right)$ (or $I=\left(t_{0}, \infty\right)$ ), $H$ is monotonically increasing in $t$ and takes all values in $\left[H_{0}, 0\right)$ for some $H_{0}<0$.

We digress to briefly discuss scale invariant expanding CMC Einstein flows. We say that this is the case if $I=(0, \infty)$ and

$$
L=1, \quad h(c t)=c^{2} h(t)
$$

for all $c>0$. Then from (2.4),

$$
K_{i j}=-t h(1)_{i j}=-\frac{1}{t} h_{i j} .
$$


Lemma 2.10. Equation (2.8) is equivalent to

$$
L=1, \quad H=-\frac{n}{t}, \quad K^{0}=0 .
$$

In this case, equations (2.2)-(2.5) are satisfied if and only if $\mathcal{E}$ is a Lorentzian cone over a Riemanniann Einstein manifold with Einstein constant $-(n-1)$, i.e.

$$
g=-d t^{2}+t^{2} h_{E i n}
$$

where $h_{\text {Ein }}$ is a Einstein metric on $X$ with Einstein constant $-(n-1)$.

Proof. The equivalence of (2.8) and (2.11) is straightforward. If (2.12) holds then it is easy to see that equations (2.2) $-(2.5)$ are satisfied. Conversely, if (2.2)-(2.5) are satisfied then (2.11) implies that $R_{i j}=-(n-1) h(1)_{i j}=-\frac{n-1}{t^{2}} h_{i j}$.

There is a more general notion of self-similarity for a vacuum Einstein solution, namely having a future-directed homothetic Killing vector field $V$. This means, in the expanding case, that $\mathcal{L}_{V} g=2 g$. If there is a compact spacelike hypersurface $X$ of constant mean curvature then $g$ must be a Lorentzian cone over a Riemannian Einstein manifold with Einstein constant $-(n-1)$; see [14] for the case $n=3$. As mentioned in the introduction, if $X$ is noncompact then there are other possibilities.

Returning to general expanding CMC Einstein flows, equation (2.5) gives

$$
\begin{aligned}
\frac{\partial H}{\partial t} & =-\triangle_{h} L+L H^{2}+L R \\
& =-\triangle_{h} L+L\left|K^{0}\right|^{2}+\frac{1}{n} L H^{2} .
\end{aligned}
$$

The maximum principle gives

$$
\frac{1}{\sup _{X}|K(t)|^{2}} \frac{\partial H}{\partial t} \leq L(t) \leq \frac{n}{H^{2}} \frac{\partial H}{\partial t} .
$$

We note in passing that if $n>1$ then (2.2) gives a formula for the normalized volume, as

$$
(-H)^{n} \operatorname{vol}(X, h(t))=\frac{n}{n-1}(-H)^{n-2} \int_{X}\left(-R^{h}+\left|K^{0}\right|^{2}\right) \operatorname{dvol}(X, h(t)) .
$$

Proposition 2.15. [16] Let $\mathcal{E}$ be an expanding $C M C$ Einstein flow. The quantity $(-H)^{n} \operatorname{vol}(X, h(t))$ is monotonically nonincreasing in $t$. It is constant in $t$ if and only if, taking $t=-\frac{n}{H}$, the Einstein flow $\mathcal{E}$ is a Lorentzian cone over a Riemannian Einstein manifold with Einstein constant $-(n-1)$.

Proof. As in [16], using (2.4) we have the pointwise identity

$$
\frac{\partial}{\partial t}\left((-H)^{n} \operatorname{dvol}(X, h)\right)=(-H)^{n+1}\left(L-\frac{n}{H^{2}} \frac{\partial H}{\partial t}\right) \operatorname{dvol}(X, h) .
$$

From (2.14), it follows that $(-H)^{n} \operatorname{dvol}(X, h(t))$ is pointwise monotonically nonincreasing in $t$, and hence $(-H)^{n} \operatorname{vol}(X, h(t))$ is monotonically nonincreasing in $t$. Alternatively, 
applying (2.13) to (2.16) gives

$$
\frac{d}{d t}\left((-H)^{n} \operatorname{vol}(X, h)\right)=-n(-H)^{n-1} \int_{X}\left|K^{0}\right|^{2} L \operatorname{dvol}(X, h) .
$$

If it is constant in $t$ then $K^{0}=0$. Taking $t=-\frac{n}{H}$, equation (2.13) gives $L=1$. As $K_{i j}=$ $\frac{1}{n} H h_{i j}=-\frac{h_{i j}}{t}$, equation (2.4) gives $h_{i j}(t)=t^{2} h_{i j}(1)$. Equation (2.5) gives $R_{i j}=-\frac{n-1}{t^{2}} h_{i j}$. The proposition follows.

Remark 2.18. Proposition 2.15 remains valid if $L$ and $h$ are locally $W^{2, p}$-regular in spacetime, and $K$ is locally $W^{1, p}$-regular in spacetime. It is also valid for an expanding CMC Einstein flow with complete finite volume time slices, provided that $L, K$ and the curvature of $h$ are bounded on compact time intervals.

2.2. Expanding CMC Einstein flows without a priori bounds. In this subsection we show that in an integral sense, for large time the rescaled Einstein flow is asymptotically scale invariant.

To motivate the result of the subsection, let us mention some properties of a scale invariant solution in the sense of Lemma 2.10,

(1) $t^{-n} \operatorname{dvol}(X, h(t))$ is constant in $t$,

(2) $t^{-2} h(t)$ is constant in $t$,

(3) $L-1=0$,

(4) $K^{0}=0$ and

(5) $R+\frac{n(n-1)}{t^{2}}=0$.

An expanding CMC Einstein flow has a limiting normalized volume measure $\mathrm{dvol}_{\infty}$; see equation (2.19) below. The results of this subsection will be true but vacuous if $\mathrm{dvol}_{\infty}=0$. Hence the results are only meaningful in the noncollapsing case.

We will introduce the rescaling of a expanding CMC Einstein flow by a parameter $s>1$, to obtain a new expanding CMC Einstein flow. Using the monotone quantity from Subsection 2.1, we show that on any fixed time interval $\left[\Lambda^{-1}, \Lambda\right]$, the properties in $(2.2)$ are asymptotically true for large $s$. More precisely, properties (1) and (2) hold asymptotically with respect to the spatial measure $\mathrm{dvol}_{\infty}$, while properties (3), (4), (5) hold asymptotically with respect to the spacetime measure $d u \mathrm{dvol}_{\infty}$.

We essentially show $C^{0}$-closeness of the rescaled flows to a scale invariant flow (relative to dvol $_{\infty}$ ) by showing that properties (1), (2) and (3) hold asymptotically. To consider a stronger statement, Lemma 2.10 says that a scale invariant Einstein flow in the sense of (2.8) has time slices with Ricci curvature $-\frac{n-1}{t^{2}} h(t)$. It is conceivable that some weak form of this statement holds asymptotically in an integral sense. We do show that the corresponding statement about scalar curvature, i.e. property (5), holds asymptotically.

To begin, taking $t=-\frac{n}{H}$, from (2.16) the measures $\left\{t^{-n} \operatorname{dvol}(X, h(t))\right\}_{t \geq t_{0}}$ are pointwise nonincreasing in $t$. They are all absolutely continuous with respect to some arbitrary smooth Riemannian measure on $X$, and their $L^{1}$-densities are pointwise nonincreasing. Put

$$
\mathrm{dvol}_{\infty}=\lim _{t \rightarrow \infty} \frac{\mathrm{d} \operatorname{vol}(X, h(t))}{t^{n}}
$$


a nonnegative absolutely continuous measure on $X$.

We give a sufficient condition for $\mathrm{dvol}_{\infty}$ to be nonzero. From (2.2),

$$
t^{2} R+n(n-1)=\left|t K^{0}\right|^{2} .
$$

Hence $R \geq-\frac{n(n-1)}{t^{2}}$ and letting $g_{X}$ range over all Riemannian metrics on $X$, we have

$$
\begin{aligned}
t^{-n} \operatorname{vol}(X, h(t)) & \geq \inf _{g_{X}}\left\{t^{-n} \operatorname{vol}\left(X, g_{X}\right): R\left(g_{X}\right) \geq-\frac{n(n-1)}{t^{2}}\right\} \\
& =\inf _{g_{X}}\left\{\operatorname{vol}\left(X, g_{X}\right): R\left(g_{X}\right) \geq-n(n-1)\right\} .
\end{aligned}
$$

It follows that

$$
\int_{X} \operatorname{dvol}_{\infty} \geq \inf \left\{\operatorname{vol}\left(X, g_{X}\right): R\left(g_{X}\right) \geq-n(n-1)\right\}
$$

If $X$ has a nonpositive $\sigma$-invariant then we obtain

$$
\int_{X} \mathrm{dvol}_{\infty} \geq\left(-\frac{\sigma(X)}{n(n-1)}\right)^{\frac{n}{2}}
$$

as was recognized in [16]. In particular, if $\operatorname{dim}(X)=3$ and $X$ contains a hyperbolic piece in its Thurston decomposition then $\sigma(X)<0$ and hence $\int_{X} \mathrm{dvol}_{\infty}>0$.

The results that follow in this subsection will be true but vacuous if $d_{v o l}$ vanishes.

Lemma 2.24. We have

$$
\lim _{t \rightarrow \infty} \frac{t^{-n} \mathrm{dvol}(X, h(t))}{\mathrm{dvol}_{\infty}}=1
$$

in $L^{1}\left(\operatorname{supp}\left(\mathrm{dvol}_{\infty}\right) ; \mathrm{dvol}_{\infty}\right)$.

Proof. As $t^{-n} \mathrm{~d} \operatorname{vol}(X, h(t))$ and $\mathrm{dvol}_{\infty}$ are absolutely continuous on $X$, the ratio $\frac{t^{-n} \operatorname{dvol}(X, h(t))}{\mathrm{dvol}_{\infty}}$ is measurable on $\operatorname{supp}\left(\mathrm{dvol}_{\infty}\right)$. As $\frac{t^{-n} \operatorname{dvol}(X, h(t))}{\mathrm{dvol}_{\infty}}$ is monotonically decreasing to 1 as $t \rightarrow \infty$, the monotone convergence theorem gives

$$
\lim _{t \rightarrow \infty} \int_{X}\left|\frac{t^{-n} \mathrm{dvol}(X, h(t))}{\mathrm{dvol}_{\infty}}-1\right| \mathrm{dvol}_{\infty}=\lim _{t \rightarrow \infty} \int_{X}\left(\frac{t^{-n} \mathrm{dvol}(X, h(t))}{\mathrm{dvol}_{\infty}}-1\right) \mathrm{dvol}_{\infty}=0 .
$$

This proves the lemma.

We now prove some integral inequalities. From (2.17), we have

$$
\begin{aligned}
& n \int_{t_{0}}^{\infty}(-H)^{n-1} \int_{X}\left|K^{0}\right|^{2} L \operatorname{dvol}(X, h(t)) d t= \\
& \left(-H\left(t_{0}\right)\right)^{n} \operatorname{vol}\left(X, h\left(t_{0}\right)\right)-\lim _{t \rightarrow \infty}(-H(t))^{n} \operatorname{vol}(X, h(t))<\infty .
\end{aligned}
$$

As

$$
t=-\frac{n}{H}
$$


we obtain

$$
\int_{t_{0}}^{\infty} \int_{X}\left|t K^{0}\right|^{2} L \frac{\mathrm{dvol}(X, h(t))}{t^{n}} \frac{d t}{t}<\infty
$$

Hence

$$
\int_{t_{0}}^{\infty} \int_{X}\left|t K^{0}\right|^{2} L \operatorname{dvol}_{\infty} \frac{d t}{t} \leq \int_{t_{0}}^{\infty} \int_{X}\left|t K^{0}\right|^{2} L \frac{\operatorname{dvol}(X, h(t))}{t^{n}} \frac{d t}{t}<\infty .
$$

Using (2.13) and (2.28), we have

$$
n(1-L)=-t^{2} \triangle_{h} L+\left|t K^{0}\right|^{2} L .
$$

The maximum principle gives $1-L \geq 0$. Then

$$
\begin{aligned}
\int_{t_{0}}^{\infty} \int_{X}|L-1| \operatorname{dvol}_{\infty} \frac{d t}{t} & =\int_{t_{0}}^{\infty} \int_{X}(1-L) \operatorname{dvol}_{\infty} \frac{d t}{t} \\
& \leq \int_{t_{0}}^{\infty} \int_{X}(1-L) \frac{\operatorname{dvol}(X, h(t))}{t^{n}} \frac{d t}{t} \\
& =\frac{1}{n} \int_{t_{0}}^{\infty} \int_{X}\left|t K^{0}\right|^{2} L \frac{\operatorname{dvol}(X, h(t))}{t^{n}} \frac{d t}{t}<\infty
\end{aligned}
$$

From (2.20) and (2.30),

$$
\begin{aligned}
\int_{t_{0}}^{\infty} \int_{X} t^{2}\left|R+\frac{n(n-1)}{t^{2}}\right| L \operatorname{dvol}_{\infty} \frac{d t}{t} & =\int_{t_{0}}^{\infty} \int_{X}\left(t^{2} R+n(n-1)\right) L \operatorname{dvol}_{\infty} \frac{d t}{t} \\
& \leq \int_{t_{0}}^{\infty} \int_{X}\left(t^{2} R+n(n-1)\right) L \frac{\operatorname{dvol}(X, h(t))}{t^{n}} \frac{d t}{t} \\
& =\int_{t_{0}}^{\infty} \int_{X}\left|t K^{0}\right|^{2} L \frac{\operatorname{dvol}(X, h(t))}{t^{n}} \frac{d t}{t}<\infty
\end{aligned}
$$

For $s>1$, the Lorentzian metric $s^{-2} g$ is isometric to

$$
g_{s}=-L^{2}(s u) d u^{2}+s^{-2} h(s u) .
$$

Hence we put

$$
\begin{array}{lc}
L_{s}(u)=L(s u), & h_{s}(u)=s^{-2} h(s u), \quad K_{s, i j}(u)=s^{-1} K_{i j}(s u), \\
H_{s}(u)=s H(s u), & K_{s, i j}^{0}(u)=s^{-1} K_{i j}^{0}(s u),\left|K^{0}\right|_{s}^{2}(u)=s^{2} K_{i j}(s u), \\
R_{s, i j}(u)=R_{i j}(s u), & R_{s}(u)=s^{2} R(s u) .
\end{array}
$$

The variable $u$ will refer to the time parameter of a rescaled Einstein flow, or a limit of such.

Proposition 2.36. Given $\Lambda>1$, we have

$$
\lim _{s \rightarrow \infty}\left(L_{s}-1\right)=\lim _{s \rightarrow \infty}\left|K^{0}\right|_{s}^{2} L_{s}=\lim _{s \rightarrow \infty}\left|R_{s}+\frac{n(n-1)}{u^{2}}\right| L_{s}=0
$$

in $L^{1}\left(\left[\Lambda^{-1}, \Lambda\right] \times X, d u \operatorname{dvol}_{\infty}\right)$. 
Proof. We prove that $\lim _{s \rightarrow \infty}\left|K^{0}\right|_{s}^{2} L_{s}=0$. The proofs for the other statements are similar, using (2.32) and (2.33).

Suppose that it is not true that $\lim _{s \rightarrow \infty}\left|K^{0}\right|_{s}^{2} L_{s}=0$ in $L^{1}\left(X \times\left[\Lambda^{-1}, \Lambda\right], \operatorname{dvol}_{\infty} d u\right)$. Then there is some $\epsilon>0$ and a sequence $\left\{s_{i}\right\}_{i=1}^{\infty}$ with $\lim _{i \rightarrow \infty} s_{i}=\infty$ and

$$
\int_{\Lambda^{-1}}^{\Lambda} \int_{X}\left|K^{0}\right|_{s_{i}}^{2} L_{s_{i}} \mathrm{dvol}_{\infty} d u \geq \epsilon
$$

After passing to a subsequence, we can assume that the intervals $\left[s_{i} \Lambda^{-1}, s_{i} \Lambda\right]$ are disjoint. Now

$$
\begin{aligned}
\int_{s_{i} \Lambda^{-1}}^{s_{i} \Lambda} \int_{X}\left|t K^{0}(t)\right|^{2} L(t) \operatorname{dvol}_{\infty} \frac{d t}{t} & =\int_{\Lambda^{-1}}^{\Lambda} \int_{X} s_{i}^{2} u^{2}\left|K^{0}\left(s_{i} u\right)\right|^{2} L\left(s_{i} u\right) \operatorname{dvol}_{\infty} \frac{d u}{u} \\
& \geq \Lambda^{-1} \int_{\Lambda^{-1}}^{\Lambda} \int_{X} s_{i}^{2}\left|K^{0}\left(s_{i} u\right)\right|^{2} L\left(s_{i} u\right) \operatorname{dvol}_{\infty} d u \\
& \geq \Lambda^{-1} \epsilon .
\end{aligned}
$$

This contradicts (2.30).

From Lemma 2.24, the volume forms of the rescaled metrics $h_{s}(u)$ approach dvol $\infty$ in an appropriate sense, as $s \rightarrow \infty$. We now look at what one can say about the rest of $h_{s}(u)$. In the scale invariant setting of Lemma 2.10, for any $s>1$, the rescaled metrics $u^{-2} h_{s}(u)$ are constant in $u$. In particular, for any $\Lambda>1$, we have $h_{s}(1)=\Lambda^{-2} h_{s}(\Lambda)$. Without assuming scale invariance, we would like to compare the nonvolume parts of $h_{s}(1)$ and $h_{s}(\Lambda)$ as $s \rightarrow \infty$. To do so, we look at their pointwise change as an element of a symmetric space.

Given $\Lambda>1, s>>1$ and $x \in X$, there is some $H_{s, \Lambda}(x) \in \operatorname{End}\left(T_{x}\right)$ such that

$$
h_{s}(x, \Lambda)=H_{s, \Lambda}(x)^{*} h_{s}(x, 1) H_{s, \Lambda}(x) .
$$

It is defined up to left multiplication by $\operatorname{Isom}\left(T_{x} X, h_{s}(x, 1)\right)$. Let $H_{s, \Lambda}^{1}(x)$ be the rescaling of $H_{s, \Lambda}(x)$ to have determinant one. After choosing an orthonormal basis of $\left(T_{x} X, h_{s}(x, 1)\right)$, the endomorphism $H_{s, \Lambda}^{1}(x)$ defines an element of the symmetric space $\mathrm{SO}(n) \backslash \mathrm{SL}(n)$ of $n \times n$ symmetric matrices with determinant one. Let $I_{n} \in \mathrm{SO}(n) \backslash \mathrm{SL}(n)$ be the basepoint represented by the identity matrix. Let $d_{\text {symm }}$ be the distance on $\mathrm{SO}(n) \backslash \mathrm{SL}(n)$, coming from the Riemannian metric given by $\langle H, H\rangle=\operatorname{Tr}\left(H^{2}\right)$ for a traceless symmetric matrix $H \in T_{I_{n}}(\mathrm{SO}(n) \backslash \mathrm{SL}(n))$.

Proposition 2.41. We have

$$
\lim _{s \rightarrow \infty} d_{s y m m}\left(H_{s, \Lambda}^{1}, I_{n}\right)=0
$$

in $L^{2}\left(X, \operatorname{dvol}_{\infty}\right)$. 
Proof. Let $M^{0}$ denote the traceless part of an $n \times n$ matrix $M$, i.e. $M^{0}=M-\frac{1}{n}(\operatorname{Tr} M) I_{n}$. From (2.4), the length of the curve $\left\{H_{s, u}^{1}(x)\right\}_{u=1}^{\Lambda}$ is

$$
\begin{aligned}
& \int_{1}^{\Lambda} \sqrt{\operatorname{Tr}\left(\left(\left(h_{s}(x, u)^{-\frac{1}{2}} \frac{\partial h_{s}(x, u)}{\partial u} h_{s}(x, u)^{-\frac{1}{2}}\right)^{0}\right)^{2}\right)} d u= \\
& 2 \int_{1}^{\Lambda}\left|K_{s}^{0}(x, u)\right| L_{s}(x, u) d u .
\end{aligned}
$$

Using the Cauchy-Schwarz inequality and the fact that $L_{s} \leq 1$,

$$
\begin{aligned}
d_{s y m m}^{2}\left(H_{s, \Lambda}^{1}(x), I_{n}\right) & \leq 4 \int_{1}^{\Lambda}\left|K_{s}^{0}\right|^{2}(x, u) L_{s}(x, u) d u \int_{1}^{\Lambda} L_{s}(x, u) d u \\
& \leq 4(\Lambda-1) \int_{1}^{\Lambda}\left|K_{s}^{0}\right|^{2}(x, u) L_{s}(x, u) d u
\end{aligned}
$$

The proposition now follows from Proposition 2.36 .

Remark 2.45. We cannot conclude from (2.42) that there is a $\mathrm{dvol}_{\infty}$-almost everywhere limit as $t \rightarrow \infty$ of $\left(\frac{\operatorname{dvol}_{\infty}}{\operatorname{dvol}_{h(t)}}\right)^{\frac{1}{n}} h(t)$. The reason is the factor of $(\Lambda-1)$ in (2.44), which prevents us from taking $\Lambda \rightarrow \infty$.

2.3. Noncollapsed type-III Einstein flows. This subsection is devoted to noncollapsed expanding CMC Einstein flows with an a priori scale invariant curvature bound. The results of this subsection are largely due to Anderson [2]. As we will need some of the results in a more general setting, we give a self-contained presentation, modulo some technical results that we quote.

Subsubsection 2.3 .1 begins with the notion of convergence for a sequence of CMC Einstein flows. We then give a compactness result for CMC Einstein flows that uniformly satisfy certain geometric bounds. We define type-III Einstein flows and obtain a compactness result for the rescalings of a noncollapsed type-III Einstein flow.

In Subsubsection 2.3.2 we assume that the noncollapsed type-III Einstien flow has a scale invariant a priori diameter bound. We show that the rescalings approach the collection of Lorentzian cones over compact Riemannian Einstein manifolds with Einstein constant $-(n-1)$. This is a straightforward generalization of the $n=3$ results in [2, Section 3]. (We use the Fischer-Moncrief normalized volume functional, whereas Anderson used a different but closely related monotonic quantity.)

Subsubsection 2.3.3 analyzes noncollapsed type-III Einstein flows without the a priori diameter bound. The result is that for large time, there is a decomposition of $X$ into a "thick part" where the rescaled flow looks like a Lorentzian cone over a compact Riemannian Einstein manifold with Einstein constant - $(n-1)$, and a "thin" part that has a $F$-structure in the sense of Cheeger-Gromov [6]. When $n$ is two or three, one can also say that after rescaling, points in the thin part are volume collapsed. The $n=3$ result was stated in [2, Section 3]; we add some detail to the arguments. 
Subsubsection 2.3.3 is not needed for the rest of the paper. Stronger conclusions in the $n=3$ case, under stronger assumptions (boundedness of Bel-Robinson energies), are in [23].

To begin, we say how we measure the pointwise size of the curvature tensor. Let $\mathcal{E}$ be an Einstein flow. Let $g$ be the corresponding Lorentzian metric. Put $e_{0}=T=\frac{1}{L} \frac{\partial}{\partial t}$, a unit timelike vector that is normal to the level sets of $t$. Let $\left\{e_{i}\right\}_{i=1}^{n}$ be an orthonormal basis for $e_{0}^{\perp}$. Put

$$
|\operatorname{Rm}|_{T}=\sqrt{\sum_{\alpha, \beta, \gamma, \delta=0}^{n} R_{\alpha \beta \gamma \delta}^{2}} .
$$

2.3.1. Limits of $C M C$ Einstein flows. Let $\mathcal{E}^{\infty}=\left(L^{\infty}, h^{\infty}, K^{\infty}\right)$ be a CMC Einstein flow on a pointed $n$-manifold $\left(X^{\infty}, x^{\infty}\right)$, with complete time slices, defined on a time interval $I^{\infty}$. For the moment, $t$ need not be the Hubble time.

Definition 2.47. The flow $\mathcal{E}^{\infty}$ is $W^{2, p}$-regular if $X^{\infty}$ is a $W^{3, p}$-manifold, $L^{\infty}$ and $h^{\infty}$ are locally $W^{2, p}$-regular in space and time, and $K^{\infty}$ is locally $W^{1, p}$-regular in space and time.

Note that the equations of Definition 2.1 make sense in this generality.

Let $\mathcal{E}^{(k)}=\left\{h^{(k)}, K^{(k)}, L^{(k)}\right\}_{k=1}^{\infty}$ be smooth CMC Einstein flows on pointed $n$-manifolds $\left\{\left(X^{(k)}, x^{(k)}\right)\right\}_{k=1}^{\infty}$, defined on time intervals $I^{(k)}$.

Definition 2.48. We say that $\lim _{k \rightarrow \infty} \mathcal{E}^{(k)}=\mathcal{E}^{\infty}$ in the pointed weak $W^{2, p}$-topology if

- Any compact interval $S \subset I^{\infty}$ is contained in $I^{(k)}$ for large $k$, and

- For any compact interval $S \subset I^{\infty}$ and any compact $n$-dimensional manifold-withboundary $W^{\infty} \subset X^{\infty}$ containing $x^{\infty}$, for large $k$ there are pointed time-independent $W^{3, p}$-regular diffeomorphisms $\phi_{S, W, k}: W^{\infty} \rightarrow W^{(k)}$ (with $W^{(k)} \subset X^{(k)}$ ) so that

$-\lim _{k \rightarrow \infty} \phi_{S, W, k}^{*} L^{(k)}=L^{\infty}$ weakly in $W^{2, p}$ on $S \times W^{\infty}$,

$-\lim _{k \rightarrow \infty} \phi_{S, W, k}^{*} h^{(k)}=h^{\infty}$ weakly in $W^{2, p}$ on $S \times W^{\infty}$ and

- $\lim _{k \rightarrow \infty} \phi_{S, W, k}^{*} K^{(k)}=K^{\infty}$ weakly in $W^{1, p}$ on $S \times W^{\infty}$.

We define pointed (norm) $C^{1, \alpha}$-convergence similarly.

Definition 2.49. Let $\mathcal{S}$ be a collection of pointed CMC Einstein flows defined on a time interval $I^{\infty}$. We say that a sequence $\left\{\mathcal{E}^{(k)}\right\}_{k=1}^{\infty}$ approaches $\mathcal{S}$ as $k \rightarrow \infty$, in the pointed weak $W^{2, p}$-topology, if for any subsequence of $\left\{\mathcal{E}^{(k)}\right\}_{k=1}^{\infty}$, there is a further subsequence that converges to an element of $\mathcal{S}$ in the pointed weak $W^{2, p}$-topology.

Definition 2.50. Let $\mathcal{S}$ be a collection of pointed CMC Einstein flows defined on a time interval $I^{\infty}$. We say that a 1-parameter family $\left\{\mathcal{E}^{(s)}\right\}_{s \in\left[s_{0}, \infty\right)}$ of pointed $C M C$ Einstein flows approaches $\mathcal{S}$, in the pointed weak $W^{2, p}$-topology, if for any sequence $\left\{s_{k}\right\}_{k=1}^{\infty}$ in $\left[s_{0}, \infty\right)$ with $\lim _{k \rightarrow \infty} s_{k}=\infty$, there is a subsequence of the flows $\left\{\mathcal{E}^{\left(s_{k}\right)}\right\}_{k=1}^{\infty}$ that converges to an element of $\mathcal{S}$ in the pointed weak $W^{2, p}$-topology.

We define "approaches $\mathcal{S}$ " in the pointed (norm) $C^{1, \alpha}$-topology similarly. The motivation for these definitions comes from how one can define convergence to a compact subset of 
a metric space, just using the notion of sequential convergence. In our applications, the relevant set $\mathcal{S}$ of Einstein flows can be taken to be sequentially compact.

The next result is essentially contained in [2, Proof of Theorem 3.1].

Proposition 2.51. Let $\left\{\mathcal{E}^{(k)}\right\}_{k=1}^{\infty}$ be a sequence of CMC Einstein flows on pointed $n$ dimensional manifolds $\left(X^{(k)}, x^{(k)}\right)$. Suppose that each $\mathcal{E}^{(k)}$ is defined on a time-interval $I^{(k)}$, on which the mean curvature $H^{(k)}$ is negative and increasing. Suppose that each $\mathcal{E}^{(k)}$ has complete time slices. Suppose that $I^{\infty} \subset \mathbb{R}$ is an interval so that for any compact interval $S \subset I^{\infty}$,

- For large $k$ we have $S \subset I^{(k)}$, and

- For large $k$, there are uniform upper bounds on $\left|H^{(k)}\right|,\left|\frac{d}{d t} H^{(k)}\right|,\left|\frac{d^{2}}{d t^{2}} H^{(k)}\right|,\left|\frac{d^{3}}{d t^{3}} H^{(k)}\right|$, $-\frac{d}{d t} \frac{1}{H^{(k)}}$ and $\left|\mathrm{Rm}^{(k)}\right|_{T}$ on $S$.

Fix $t_{0} \in I^{\infty}$. Suppose that there is some $v_{0}>0$ so that for all large $k$, the time- $t_{0}$ unit ball satisfies $\operatorname{vol}\left(B_{h^{(k)}\left(t_{0}\right)}\left(x^{(k)}, 1\right)\right) \geq v_{0}$. Then after passing to a subsequence, there is a limit $\lim _{k \rightarrow \infty} \mathcal{E}^{(k)}=\mathcal{E}^{\infty}$ in the pointed weak $W^{2, p}$-topology and the pointed $C^{1, \alpha}$-topology. The limit flow $\mathcal{E}^{\infty}$ is defined on a pointed $n$-manifold $\left(X^{\infty}, x^{\infty}\right)$, and on the time interval $I^{\infty}$. Its time slices are complete.

If for each compact interval $S \subset I^{\infty}$, there is some $C_{S}<\infty$ such that $\left|K^{(k)}\right|^{2} \leq C_{S} \frac{d H^{(k)}}{d t}$ for all large $k$, on the time interval $S$, then the limiting lapse function $L^{\infty}$ is positive.

Proof. On any compact interval $S \subset I^{\infty}$, the bounds on $H^{(k)}$ and $\left|\mathrm{Rm}^{(k)}\right|_{T}$ give bounds on $\left|K^{(k)}\right|$ for large $k$ [2, Proposition 2.2], and hence on the curvature of $h^{(k)}$. From (2.14), there is a uniform upper bound on the lapse functions $L^{(k)}$. Using (2.13) and taking $t$ derivatives of it, there are $W^{2, p}$-bounds on the $L^{(k)}$ 's; see [2, p. 551] and [9, Section 3]. One also has first derivative bounds on $K$. In all, one obtains $W^{2, p}$-bounds on $\left\{\mathcal{E}^{(k)}\right\}_{k=1}^{\infty}$ over the time interval $S$; c.f. [9, Theorem 3.1]

Using the lower volume bound, after passing to a subsequence of the pointed Riemannian manifolds $\left\{\left(X^{(k)}, x^{(k)}, h^{(k)}\left(t_{0}\right)\right)\right\}_{k=1}^{\infty}$ there is a pointed $W^{3, p}$-regular limit manifold $\left(X^{\infty}, x^{\infty}\right)$ with a complete $W^{2, p}$-regular limit Riemannian metric $h^{\infty}\left(t_{0}\right)$. Let $W^{\infty} \subset X^{\infty}$ be a compact $n$-dimensional manifold-with-boundary containing $x^{\infty}$ and let $\phi_{W, k}: W^{\infty} \rightarrow W^{(k)}$ be the comparison diffeomorphisms inherent in forming $X^{\infty}$. Put

$$
\phi_{S, W, k}=\left(\operatorname{Id}_{S} \times \phi_{W, k}\right):\left(S \times W^{\infty}\right) \rightarrow\left(S \times X^{(k)}\right) .
$$

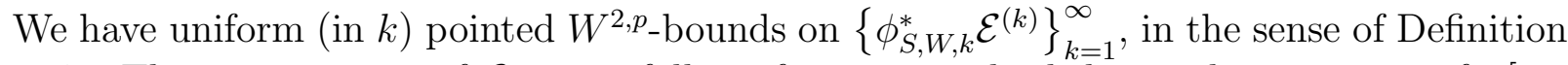
2.47. The construction of $\mathcal{E}^{\infty}$ now follows from a standard diagonal argument; c.f. [18, Section 2].

The uniform bounds on $\left|K^{(k)}\right|$ give uniform multiplicative bounds on the distance distortion when going from time $t_{0}$ to another time $t \in I^{\infty}$, from which the completeness of $\left(X^{\infty}, h^{\infty}(t)\right)$ follows.

If $\left|K^{(k)}\right|^{2} \leq C_{S} \frac{d H^{(k)}}{d t}$ then (2.14) implies that $L^{(k)} \geq C_{S}^{-1}$ on the time interval $S$. Hence $L^{\infty} \geq C_{S}^{-1}$ on $S$. 
We take $t=-\frac{n}{H}$.

Definition 2.53. A type-III Einstein flow is an expanding CMC Einstein flow for which there is some $C<\infty$ so that $|\mathrm{Rm}|_{T} \leq C t^{-2}$.

Recall the rescaling from (2.35). We write the rescaled Einstein flow as $\mathcal{E}_{s}$. It is also type-III, with the same constant $C$.

Corollary 2.54. Let $\mathcal{E}$ be a type-III Einstein flow on an n-dimensional manifold X. Suppose that it is defined on a time-interval $\left[t_{0}, \infty\right)$ with $t_{0}>0$, and has complete time slices. Let $\left\{t_{i}\right\}_{i=1}^{\infty}$ be a sequence in $\left[t_{0}, \infty\right)$ with $\lim _{i \rightarrow \infty} t_{i}=\infty$ and let $\left\{x_{i}\right\}_{i=1}^{\infty}$ be a sequence in $X$ with the property that vol $\left(B_{h\left(t_{i}\right)}\left(x_{i}, t_{i}\right)\right) \geq v_{0} t_{i}^{n}$ for large $i$, and some $v_{0}>0$. Then after passing to a subsequence, which we relabel as $\left\{t_{i}\right\}_{i=1}^{\infty}$ and $\left\{x_{i}\right\}_{i=1}^{\infty}$, there is a limit $\lim _{i \rightarrow \infty} \mathcal{E}_{t_{i}}=\mathcal{E}^{\infty}$ in the pointed weak $W^{2, p}$-topology and the pointed $C^{1, \alpha}$-topology. The limit flow $\mathcal{E}^{\infty}$ is defined on the time interval $(0, \infty)$. Its time slices $\left\{\left(X^{\infty}, h^{\infty}(u)\right)\right\}_{u>0}$ are complete. Its lapse function $L^{\infty}$ is uniformly bounded below by a positive constant.

Proof. Put $I^{(i)}=\left[t_{0} / t_{i}, \infty\right), I^{\infty}=(0, \infty)$ and $\mathcal{E}^{(i)}=\mathcal{E}_{t_{i}}^{(i)}$. The existence of $\mathcal{E}^{\infty}$ follows from Proposition 2.51. From the proof of Proposition [2.51, on any compact interval $S \subset I^{\infty}$ there is a bound $\left|K^{(i)}\right|^{2} \leq$ const. $t^{-2}$ that is uniform in $i$. As $\frac{d H^{(i)}}{d t}=\frac{n}{t^{2}}$, Proposition 2.51 implies that $L^{\infty}>0$. From its proof, $L^{\infty}$ is uniformly bounded below by a positive constant.

2.3.2. Noncollapsed Type-III Einstein flows with a diameter bound. In the rest of this section, we will only consider type-III Einstein flows. In this subsubsection, we make the following assumption.

Assumption 2.55. There is some $D<\infty$ so that for all $t$, we have $\operatorname{diam}(X, h(t)) \leq D t$.

Let $\mathcal{S}$ be the collection of Einstein flows that generate Lorentzian cones over compact $n$-dimensional Riemannian Einstein manifolds with Einstein constant $-(n-1)$. They are defined on the time interval $(0, \infty)$.

Proposition 2.56. [2] Suppose that a type-III Einstein flow $\mathcal{E}$ satisfies Assumption [2.55, with $\lim _{t \rightarrow \infty} t^{-n} \operatorname{vol}(X, h(t))>0$. Then as $s \rightarrow \infty$, the rescaled flows $\mathcal{E}_{s}$ approach $\mathcal{S}$ in the weak $W^{2, p}$-topology and $C^{1, \alpha}$-topology.

Proof. Let $\left\{s_{i}\right\}_{i=1}^{\infty}$ satisfy $\lim _{i \rightarrow \infty} s_{i}=\infty$. Pick arbitrary basepoints $x_{i} \in X$. From the upper diameter bound and the positive lower volume bound on $\left(X, h_{s_{i}}(1)\right)$, the BishopGromov inequality gives a $v_{0}>0$ so that for each $i$, we have $\operatorname{vol}\left(B_{h_{s_{i}}(1)}\left(x_{i}, 1\right)\right) \geq v_{0}$. Corollary 2.54 now gives a subsequential limit Einstein flow $\mathcal{E}^{\infty}$, which a priori is $W^{2, p_{-}}$ regular in the sense of Definition 2.47. Because of the diameter bounds, $X^{\infty}$ is compact. The monotonicity of $t^{-n} \operatorname{vol}(X, h(t))$ implies that $u^{-n} \operatorname{vol}\left(X^{\infty}, h^{\infty}(u)\right)$ is constant in $u$. By Proposition 2.15 and Remark 2.18, $\mathcal{E}^{\infty} \in \mathcal{S}$. This proves the proposition.

The lower volume bound in Proposition 2.56 is guaranteed when the topology of $X$ is such that it cannot collapse with bounded curvature and bounded diameter. For example, it suffices that $X$ have a nonzero characteristic number or a nonvanishing simplicial volume. 
The conclusion of Proposition 2.56 implies that $X$ carries an Einstein metric with Einstein constant $-(n-1)$.

In three dimensions, if $X$ admits a hyperbolic metric then it has positive simplicial volume and cannot collapse with bounded curvature and bounded diameter. An Einstein three-manifold with Einstein constant -2 is hyperbolic.

Corollary 2.57. Suppose that a three dimensional type-III Einstein flow $\mathcal{E}$ satisfies Assumption 2.55, with $\lim _{t \rightarrow \infty} t^{-3} \operatorname{vol}(X, h(t))>0$. Let $\widetilde{\mathcal{E}}$ denote the pullback Einstein flow on the universal cover $\widetilde{X}$. For $s>0$, choose $\widetilde{x}_{s} \in \widetilde{X}$. Then as $s \rightarrow \infty$, the pointed rescaled flows $\left(\widetilde{\mathcal{E}}_{s}, \widetilde{x}_{s}\right)$ approach the flat Milne solution with basepoint $\left(1, \widetilde{x}_{\infty}\right) \in(0, \infty) \times H^{3}$, in the pointed weak $W^{2, p}$-topology and the pointed $C^{1, \alpha}$-topology.

Remark 2.58. The notion of convergence in Proposition 2.56 is up to $s$-dependent diffeomorphisms. For this reason, Proposition 2.56 does not imply that $\lim _{s \rightarrow \infty} h_{s}(\cdot)$ exists as a metric. As Proposition 2.56 does give regions that are arbitrarily close to Lorentzian cones in $\mathcal{S}$, a stability result for Lorentzian cones would imply that that $\lim _{s \rightarrow \infty} h_{s}(\cdot)$ exists as a metric. When $n=3$, the stability result of [3] needs, in particular, $H^{3}$-closeness of $h_{s}(1)$ to the hyperbolic metric on $X$. From Proposition 2.56 we only get weak $W^{2, p}$-closeness or $C^{1, \alpha}$-closeness. If we strengthen the type-III assumption to include $|\nabla \mathrm{Rm}|_{T} \leq C t^{-3}$ and $|\nabla \nabla \mathrm{Rm}|_{T} \leq C t^{-4}$ then we will get $C^{3, \alpha}$-closeness and the stability result will apply.

2.3.3. Noncollapsed Type-III Einstein flows without a diameter bound. In this subsubsection we remove the diameter assumption in Subsubsection 2.3.2.

Let $\mathcal{S}$ be the collection of Einstein flows that generate Lorentzian cones over finite volume complete connected pointed $n$-dimensional Riemannian Einstein manifolds with Einstein constant $-(n-1)$. They are defined on the time interval $(0, \infty)$.

Proposition 2.59. Let $\mathcal{E}$ be a type-III Einstein flow with time slices diffeomorphic to a compact connected $n$-dimensional manifold $X$. Given $v>0$, there is some $N_{v} \in \mathbb{N}$ so that for all $t \geq t_{0}$, there is a set $\left\{x_{t, j}\right\}_{j=1}^{N_{t}^{\prime}}$ in $X$, with $N_{t}^{\prime} \leq N_{v}$, such that

- Each $x \in X-\bigcup_{j=1}^{N_{t}^{\prime}} B_{h(t)}\left(x_{t, j}, 2 t\right)$ has $t^{-n} \operatorname{vol}\left(B_{h(t)}(x, t)\right)<v$. Here $B_{h(t)}(x, t) d e-$ notes the ball of radius $t$ around $x$ with respect to the metric $h(t)$.

- Let $\left\{t_{i}\right\}_{i=1}^{\infty}$ be a sequence tending to infinity such that $\left\{x_{t_{i}, j}\right\}_{j=1}^{N_{t_{i}}^{\prime}}$ is nonempty for each $i$. Let $x_{i}$ be a choice of an element of $\left\{x_{t_{i}, j}\right\}_{j=1}^{N_{t_{i}}^{\prime}}$ for each $i$. Then as $i \rightarrow \infty$, the pointed rescaled flows $\left(\mathcal{E}_{t_{i}}, x_{i}\right)$ approach $\mathcal{S}$ in the pointed weak $W^{2, p}$-topology and the pointed $C^{1, \alpha}$-topology.

Proof. Define the $v$-thick part of $(X, h(t))$ by

$$
X_{v-\text { thick }, h(t)}=\left\{x \in X: t^{-n} \operatorname{vol}\left(B_{h(t)}(x, t)\right) \geq v\right\} .
$$

If $X_{v-t h i c k, h(t)} \neq \emptyset$, choose a maximal collection of points $\left\{x_{t, j}\right\}$ in $X_{v-t h i c k, h(t)}$ so that the balls $B_{h(t)}\left(x_{t, j}, t\right)$ are disjoint. From volume monotonicity, there is some $V_{0}<\infty$ so that for all $t \geq t_{0}$, we have $t^{-n} \operatorname{vol}(X, h(t)) \leq V_{0}$. Hence the number of points in the collection is bounded above by $N=\frac{V_{0}}{v}$. The first conclusion of Proposition 2.59 follows. 
Given the sequence $\left\{t_{i}\right\}_{i=1}^{\infty}$ tending to infinity, after passing to a subsequence, from Corollary 2.54 the rescaled pointed flows $\left\{\left(\mathcal{E}_{t_{i}}, x_{i}\right)\right\}$ converge to a pointed $W^{2, p}$-regular Einstein flow $\left(\mathcal{E}^{\infty}, x^{\infty}\right)$ with complete time slices $\left(X^{\infty}, h^{\infty}(u)\right)$ of constant mean curvature $-\frac{n}{u}$, defined for $u \in(0, \infty)$.

To show that $\left(\mathcal{E}^{\infty}, x^{\infty}\right)$ lies in $\mathcal{S}$, we claim first that its lapse function $L^{\infty}$ is identically one. Suppose not. From (2.31), the lapse $L$ for $\mathcal{E}$ is bounded above by one. Hence $L^{\infty}$ is also bounded above by one. Suppose that $L^{\infty} \neq 1$. Then there are a compact set $K^{\infty} \subset X^{\infty}$, a time interval $\left[u_{1}, u_{2}\right] \subset(0, \infty)$ and a number $\epsilon>0$ so that

$$
\int_{u_{1}}^{u_{2}} \int_{K^{\infty}}\left(1-L^{\infty}\right) \frac{\mathrm{d} \operatorname{vol}\left(X^{\infty}, h^{\infty}(u)\right)}{u^{n}} \frac{d u}{u}>\epsilon .
$$

It follows that for large $i$, there are compact subsets $K_{i} \subset X$ so that

$$
\int_{t_{i} u_{1}}^{t_{i} u_{2}} \int_{K_{i}}(1-L) \frac{\mathrm{d} \operatorname{vol}(X, h(t))}{t^{n}} \frac{d t}{t}>\frac{\epsilon}{2} .
$$

From the set of intervals $\left\{\left[t_{i} u_{1}, t_{i} u_{2}\right]\right\}_{i=1}^{\infty}$, we can extract a subset consisting of an infinite number of disjoint intervals. Then (2.62) gives a contradiction to the fact from (2.32) that

$$
\int_{t_{0}}^{\infty} \int_{X}(1-L) \frac{\mathrm{d} \operatorname{vol}(X, h(t))}{t^{n}} \frac{d t}{t}<\infty .
$$

Hence $L^{\infty}=1$. A similar argument, using (2.29), shows that $\left|K^{0, \infty}\right|^{2} L^{\infty}=0$. Then from Lemma 2.10, the limit flow $\mathcal{E}^{\infty}$ lies in $\mathcal{S}$. This proves the proposition.

Remark 2.64. From [7, Theorem 0.1] and [8, Theorem 4.7], there is some $v_{0}=v_{0}(n, C)>0$ so that for all large $t$, the complement of the $v_{0}$-thick set $X_{v_{0}-t h i c k, h(t)}$ is part of an open subset of $X$ with an $F$-structure. (Here $C$ is the constant from Definition 2.53.) In particular, if $X$ does not carry an $F$-structure then $X_{v_{0}-t h i c k, h(t)}$ is nonempty for all large $t$. For example, it suffices that $X$ have a nonzero Euler characteristic or a nonvanishing simplicial volume, e.g. if $\operatorname{dim}(X)=3$ that $X$ has a hyperbolic piece in its Thurston decomposition.

2.3.4. Dimensions two and three. If $n$ is two or three then a finite volume complete Riemannian manifold with Ric $=-(n-1) g$ is hyperbolic, i.e. has constant sectional curvature -1 . There is a positive lower bound on the volumes of such manifolds.

For $n \in\{2,3\}$, let $\mathcal{S}$ now be the collection of Einstein flows that generate flat Lorentzian cones over finite volume complete connected pointed $n$-dimensional hyperbolic manifolds.

Proposition 2.65. Let $\mathcal{E}$ be a type-III Einstein flow with time slices diffeomorphic to a compact connected $n$-dimensional manifold $X$, where $n \in\{2,3\}$. Then there are a number $N \in \mathbb{N}$ and a function $\sigma:\left[t_{0}, \infty\right) \rightarrow(0, \infty)$ with $\lim _{t \rightarrow \infty} \sigma(t)=0$ so that for all $t \geq t_{0}$, there is a set $\left\{x_{t, j}\right\}_{j=1}^{N_{t}^{\prime}}$ in $X$, with $N_{t}^{\prime} \leq N$, such that

- Each $x \in X-\bigcup_{j=1}^{N_{t}^{\prime}} B_{h(t)}\left(x_{t, j}, \frac{t}{\sigma(t)}\right)$ has $t^{-n} \operatorname{vol}\left(B_{h(t)}(x, t)\right)<\sigma(t)$. 
- Let $\left\{t_{i}\right\}_{i=1}^{\infty}$ be a sequence tending to infinity such that $\left\{x_{t_{i}, j}\right\}_{j=1}^{N_{t_{i}}^{\prime}}$ is nonempty for each $i$. Let $x_{i}$ be a choice of an element of $\left\{x_{t_{i}, j}\right\}_{j=1}^{N_{t_{i}}^{\prime}}$ for each $i$. Then as $i \rightarrow \infty$, the pointed rescaled flows $\left(\mathcal{E}_{t_{i}}, x_{i}\right)$ approach $\mathcal{S}$ in the pointed weak $W^{2, p}$-topology and the pointed $C^{1, \alpha}$-topology.

Proof. Using the Margulis lemma and pointed compactness, there is some $v_{0}>0$ so that for all sufficiently small $v>0$, there is some $D(v)<\infty$ with the following property. If $(Z, h)$ is a finite volume complete connected $n$-dimensional hyperbolic manifold, $n \in\{2,3\}$, then $Z_{v-t h i c k, h}$ is contained in the $D(v)$-neighborhood of $Z_{2 v_{0}-t h i c k, h}$.

Consequently, we can carry out the proof of Proposition 2.59] while letting $v$ go to zero, but keeping basepoints $\left\{x_{t, j}\right\}_{j=1}^{N_{t}^{\prime}}$ within $X_{v_{0}-t h i c k, h(t)}$. The proposition follows.

Remark 2.66. There is a possible redundancy in the choice of basepoints $\left\{x_{t, j}\right\}_{j=1}^{N_{t}^{\prime}}$ in Proposition 2.65. In the second conclusion of the proposition, if $x_{i}=x_{t_{i}, j_{i}}$ and $x_{i}^{\prime}=x_{t_{i}, j_{i}^{\prime}}$ are choices of basepoints with $d_{h\left(t_{i}\right)}\left(x_{i}, x_{i}^{\prime}\right)=O\left(t_{i}\right)$ then they will give rise to the same element of $\mathcal{S}$, up to a change of basepoint. After eliminating this redundancy, we can say that for large $t$, there is a decomposition of $\left(X, \frac{h(t)}{t^{2}}\right)$ into an almost-hyperbolic part and a locally collapsing part.

We do not claim that as $t \rightarrow \infty$, the volume of the almost-hyperbolic part approaches $\lim _{t \rightarrow \infty} t^{-n} \operatorname{vol}(X, h(t))$. That is, it is conceivable that there is a substantial part of the volume in the locally collapsing part of $(X, h(t))$.

\section{Einstein FLOWS ON ÉtAle GROUPOIDS}

This section contains the results about collapsed type-III Einstein flows. The convergence results are phrased in terms of Einstein flows on étale groupoids. We refer to [22, Section 3] for an overview, aimed at geometers, of the use of groupoids in collapsing theory. More details appear in [21, Section 5].

In Subsection 3.1 we define Einstein flows on étale groupoids. We extend the results of Subsubsection 2.3 .1 by removing the lower volume bound assumption. As an immediate application, we strengthen the convergence result of Subsection 2.2 when the Einstein flow is type-III. Namely, for any point $x \in X$ with $\operatorname{dvol}_{\infty}(x) \neq 0$, the rescaled Einstein flows around $x$ converge in the pointed sense to Lorentzian cones over (possibly collapsed) Riemannian Einstein metrics with Einstein constant $-(n-1)$.

From Subsection 3.1, after performing rescalings on a type-III Einstein flow $\mathcal{E}$, we can extract subsequential limit Einstein flows that live on étale groupoids. In the rest of the section, we restrict to the case $n=3$. We also assume a scale invariant a priori diameter bound on $\mathcal{E}$. The goal is to show that there are arbitrarily large future time intervals on which $\mathcal{E}$ is modelled, in a scale invariant way, by one of a few homothety-invariant homogeneous Einstein flows, depending on the Thurston type of $X$.

The dimension of the orbit space of the étale groupoid is the same as the dimension of the Gromov-Hausdorff limit of the rescaled time slices. The case when the dimension is three was covered in Subsubsection 2.3.2. The cases when the orbit space has dimension 
zero, one or two are covering in Subsections 3.2, 3.3 and 3.4, respectively. More detailed descriptions are at the beginnings of the subsections.

To summarize the relation between type-III flows (with a scale invariant diameter bound) and topology, we recall the notion of the Thurston type of a compact 3-manifold [27]. This is a topological notion, i.e. we do not only consider locally homogeneous metrics.

(1) If $\mathcal{E}$ has a rescaling limit flow with a zero dimensional orbit space then $X$ has Thurston type $\mathbb{R}^{3}$ or Nil.

(2) If $X$ has Thurston type $S o l$ then any rescaling limit flow of $\mathcal{E}$ has a one dimensional orbit space. Conversely, if a rescaling limit flow of $\mathcal{E}$ has a one dimensional orbit space then $X$ has Thurston type $S o l, \mathbb{R}^{3}$ or $N i l$.

(3) If $X$ has Thurston type $H^{2} \times \mathbb{R}$ or $\widehat{\operatorname{SL}(2, \mathbb{R})}$ then any rescaling limit flow of $\mathcal{E}$ has a two dimensional orbit space. Conversely, if a rescaling limit flow of $\mathcal{E}$ has a two dimensional orbit space then $X$ has Thurston type $H^{2} \times \mathbb{R}, \widehat{\mathrm{SL}(2, \mathbb{R})}, \mathbb{R}^{3}$ or $N i l$. One can speculate that in fact, $X$ must have Thurston type $H^{2} \times \mathbb{R}$ or $\widetilde{\mathrm{SL}(2, \mathbb{R})}$; this is true when Proposition 3.49 applies.

(4) If $X$ has Thurston type $H^{3}$ then any rescaling limit flow of $\mathcal{E}$ has a three dimensional orbit space. Conversely, if a rescaling limit flow of $\mathcal{E}$ has a three dimensional orbit space then $X$ has Thurston type $H^{3}$.

If $X$ has Thurston type $\mathbb{R}^{3}$ then the orbit space of a rescaling limit flow could be zero dimensional (as happens for a quotient of a generic Kasner solution) or one dimensional (as happens for a quotient of the Taub-flat spacetime). The same is true for Thurston type Nil.

3.1. Collapsing limits of expanding CMC Einstein flows. In what follows, $\mathcal{X}$ will denote a closed effective Hausdorff étale groupoid [21, Section 5]. We will loosely refer to it just as an étale groupoid.

Definition 3.1. Let $I$ be an interval in $\mathbb{R}$. An Einstein flow $\mathcal{E}$ on an $n$-dimensional étale groupoid $\mathcal{X}$ is given by a family of nonnegative functions $\{L(t)\}_{t \in I}$ on $\mathcal{X}$, a family of Riemannian metrics $\{h(t)\}_{t \in I}$ on $\mathcal{X}$, and a family of symmetric covariant 2 -tensor fields $\{K(t)\}_{t \in I}$ on $\mathcal{X}$ so that equations (2.2)-(2.5) are satisfied.

We can talk about $\mathcal{E}$ being $W^{2, p}$-regular as in Definition 2.47. The $W^{2, p}$-norms can be defined using integration over the orbit space, as in [19, Section 2.6].

An expanding CMC Einstein flow on an étale groupoid $\mathcal{X}$ is defined as in Definition 2.7.

The definition of convergence of Riemannian groupoids is given in [21, Definition 5.8]. Let $\left\{\mathcal{E}^{(k)}\right\}_{k=1}^{\infty}$ be CMC Einstein flows on pointed étale groupoids. If $\mathcal{E}^{\infty}$ is a CMC Einstein flow on a pointed étale groupoid, whose time slices have metrically complete orbit spaces, then we define pointed weak $W^{2, p}$-convergence of $\left\{\mathcal{E}^{(k)}\right\}_{k=1}^{\infty}$ to $\mathcal{E}^{\infty}$ by the corresponding spacetime extension, as in Definition 2.48. Let $\mathcal{S}$ be a set of CMC Einstein flows on pointed étale groupoids, whose time slices have metrically complete orbit spaces. If $\left\{\mathcal{E}^{(k)}\right\}_{k=1}^{\infty}$ are pointed CMC Einstein flows on étale groupoids then as in Definition 2.49, we can talk about $\left\{\mathcal{E}^{(k)}\right\}_{k=1}^{\infty}$ approaching $\mathcal{S}$ as $k \rightarrow \infty$. If $\left\{\mathcal{E}^{(s)}\right\}_{s \in\left[s_{0}, \infty\right)}$ is a 1-parameter family of 
pointed CMC Einstein flows on étale groupoids then as in Definition 2.50, we can talk about $\left\{\mathcal{E}^{(s)}\right\}_{s \in\left[s_{0}, \infty\right)}$ approaching $\mathcal{S}$ as $s \rightarrow \infty$.

Proposition 3.2. Let $\left\{\mathcal{E}^{(k)}\right\}_{k=1}^{\infty}$ be sequence of CMC Einstein flows on pointed $n$-dimensional manifolds $\left(X^{(k)}, x^{(k)}\right)$. Suppose that $\mathcal{E}^{(k)}$ is defined on a time-interval $I^{(k)}$, on which the mean curvature $H^{(k)}$ is negative and increasing. Suppose that $\mathcal{E}^{(k)}$ has complete time slices. Suppose that $I^{\infty} \subset \mathbb{R}$ is an interval so that for any compact interval $S \subset I^{\infty}$,

- For large $k$ we have $S \subset I^{(k)}$, and

- For large $k$, there are uniform upper bounds on $\left|H^{(k)}\right|,\left|\frac{d}{d t} H^{(k)}\right|,\left|\frac{d^{2}}{d t^{2}} H^{(k)}\right|,\left|\frac{d^{3}}{d t^{3}} H^{(k)}\right|$ $-\frac{d}{d t} \frac{1}{H^{(k)}}$ and $\left|\mathrm{Rm}^{(k)}\right|_{T}$ on $S$.

Then after passing to a subsequence, there is a limit $\lim _{k \rightarrow \infty} \mathcal{E}^{(k)}=\mathcal{E}^{\infty}$ in the pointed weak $W^{2, p}$-topology and the pointed $C^{1, \alpha}$-topology. The limit flow $\mathcal{E}^{\infty}$ is defined on a pointed $n$-dimensional étale groupoid $\left(\mathcal{X}^{\infty}, \mathcal{O}^{\infty}\right)$, and on the time interval $I^{\infty}$. The time slices have metrically complete orbit spaces.

If for each compact interval $S \subset I^{\infty}$, there is some $C_{S}<\infty$ such that $\left|K^{(k)}\right|^{2} \leq C_{S} \frac{d H^{(k)}}{d t}$ for all large $k$, on the time interval $S$, then the limiting lapse function $L^{\infty}$ is positive.

Proof. The proof is similar to that of Proposition 2.51, On any compact interval $S \subset$ $I^{\infty}$, the uniform bounds on $H^{(k)}$ and $\left|\mathrm{Rm}^{(k)}\right|_{T}$ give uniform bounds on $\left|K^{(k)}\right|$ for large $k$ [2, Proposition 2.2], and hence on the curvature of $h^{(k)}$. Choose $t_{0} \in I^{\infty}$. As in [21, Proposition 5.9], after passing to a subsequence the pointed Riemannian manifolds $\left\{\left(X^{(k)}, x^{(k)}, h^{(k)}\right)\right\}_{k=1}^{\infty}$ converge in the pointed weak $W^{2, p}$-topology to a pointed Riemannian groupoid $\left(\mathcal{X}^{\infty}, \mathcal{O}^{\infty}, h^{\infty}\left(t_{0}\right)\right)$ whose orbit space is metrically complete. (The smooth convergence in [21, Proposition 5.9] gets replaced by pointed weak $W^{2, p}$-convergence.) Given this, the construction of a limit on the time interval $I^{\infty}$ is similar to that in the proof of Proposition 2.51.

Remark 3.3. There is an analog of Proposition 3.2 when $\mathcal{E}^{(k)}$ is a CMC Einstein flow on an $n$-dimensional étale groupoid.

The unit space $\mathcal{X}_{(0)}^{\infty}$ of the étale groupoid $\mathcal{X}^{\infty}$ carries a locally constant sheaf $\mathfrak{n}$ of finitedimensional Lie algebras, which act as germs of Killing vector fields on $\left(\mathcal{X}_{(0)}^{\infty}, h^{\infty}(t)\right)$. For any $t$, the Riemannian groupoid $\left(\mathcal{X}_{(0)}^{\infty}, h^{\infty}(t)\right)$ is a limit of Riemannian manifolds with bounded curvature; hence the Lie algebras are nilpotent.

We take $t=-\frac{n}{H}$.

Corollary 3.4. Let $\mathcal{E}$ be a type-III Einstein flow on a pointed $n$-dimensional manifold $(X, x)$. Suppose that it is defined on a time-interval $\left[t_{0}, \infty\right)$ with $t_{0}>0$, and has complete time slices. Then for any sequence $\left\{t_{i}\right\}_{i=1}^{\infty}$ in $\left[t_{0}, \infty\right)$ with $\lim _{i \rightarrow \infty} t_{i}=\infty$, after passing to a subsequence, which we relabel as $\left\{t_{i}\right\}_{i=1}^{\infty}$, there is a limit $\lim _{i \rightarrow \infty} \mathcal{E}_{t_{i}}=\mathcal{E}^{\infty}$ in the pointed weak $W^{2, p}$-topology and the pointed $C^{1, \alpha}$-topology. The limit flow $\mathcal{E}^{\infty}$ exists on a pointed étale groupoid $\left(\mathcal{X}^{\infty}, \mathcal{O}^{\infty}\right)$ and is defined on the time interval $(0, \infty)$. The orbit spaces of 
its time slices are metrically complete. Its lapse function $L^{\infty}$ is uniformly bounded below by a positive constant.

Proof. Given Proposition 3.2, the proof is similar to that of Corollary 2.54,

Recall the definition of $\mathrm{dvol}_{\infty}$ from (2.19).

Proposition 3.5. Let $\mathcal{S}$ denote the collection of Einstein flows that are Lorentzian cones over Riemannian Einstein metrics on étale groupoids, with Einstein constant $-(n-1)$. Under the hypotheses of Corollary 3.4, suppose that the basepoint $x$ is such that $\mathrm{dvol}_{\infty}(x) \neq$ 0 . Then as $t \rightarrow \infty$, the rescaled flows $\left\{\mathcal{E}_{t}\right\}_{t=1}^{\infty}$ approach $\mathcal{S}$.

Proof. With reference to Corollary 3.4, we must show that $\mathcal{E}^{\infty}$ describes a Lorentzian cone over an Einstein metric on $\mathcal{X}^{\infty}$, with Einstein constant $-(n-1)$. From (2.16), we have

$$
\frac{\partial}{\partial t} \ln \frac{\mathrm{dvol}_{\infty}}{t^{-n} \mathrm{dvol}_{t}}=\frac{n}{t}(1-L)
$$

Hence

$$
\int_{t_{0}}^{\infty}(1-L(x, t)) \frac{d t}{t}<\infty .
$$

(Recall that $L \leq 1$.) Then $L_{\infty}(x, u)=1$ for all $u \in(0, \infty)$. Equation (2.31) (with $t$ replaced by $u$ ), along with elliptic regularity, the fact that $g_{\infty}$ is locally $C^{1, \alpha}$-regular and the fact that $K_{\infty}^{0}$ is locally $C^{\alpha}$-regular, implies that $L_{\infty}(x, u)$ is locally $C^{2, \alpha}$-regular in $x$. We can apply the strong maximum principle to (2.31) on the unit space of $\mathcal{X}^{\infty}$ to obtain that $L_{\infty}=1$ and $K_{\infty}^{0}=0$. The proposition follows from Lemma 2.10 .

Corollary 3.8. Under the hypotheses of Proposition [3.5, for any $C<\infty$ we have the following asymptotics as $t \rightarrow \infty$ :

(1) The supremum of $|L-1|$ on the time-t ball $B_{h(t)}(x, C t)$ is o(t $\left.t^{0}\right)$.

(2) The supremum of $\left|K^{0}\right|$ on the time-t ball $B_{h(t)}(x, C t)$ is o(t-1).

Proof. The corollary follows from the $C^{\alpha}$ convergence of $L$ and $K^{0}$, after rescaling.

Remark 3.9. We cannot conclude that the Einstein flow is noncollapsing around $x$, in the sense of volumes of metric balls. Although the volume form is noncollapsing in a neighborhood of $x$ in $X$, there is not enough control on the change of distances to deduce noncollapsing of a time- $t$ metric ball around $x$ of radius comparable to $t$, as $t \rightarrow \infty$. $A$ priori, the rescaled spatial geometry around $x$ could resemble that of a point going out the end of a hyperbolic cusp, for example, while the diameter of a fixed neighborhood $U \subset X$ of $x$ increases faster than $O(t)$ so as to keep $\operatorname{vol}(U) \geq$ const. $t^{n}$.

In what follows, we will assume that $n=3$, and also make the following assumption.

Assumption 3.10. There is some $D<\infty$ so that for all $t$, we have $\operatorname{diam}(X, h(t)) \leq D t$. 
The reason for Assumption 3.10 is that we will want to apply monotonicity arguments to limit spaces, and will need to know that they have compact spatial hypersurfaces. This is ensured by Assumption 3.10

Because of the bounded diameter assumption, in what follows we will not have to choose basepoints. Assumption 3.10, along with the type-III assumption, implies that the Thurston decomposition of $X$ consists of a single topological type [22, Proposition 3.5]. We will also assume that $X$ is aspherical, i.e. has a contractible cover. Then the relevant Thurston types are $\mathbb{R}^{3}, H^{3}, H^{2} \times \mathbb{R}, \widehat{\mathrm{SL}(2, \mathbb{R})}$, Nil and Sol. From [22, Lemma 6.1], the étale groupoid $\mathcal{X}^{\infty}$ of Corollary 3.4 is locally free.

3.2. Zero dimensional orbit space. In this subsection we look at the case when there is a rescaling limit that is an Einstein flow on an étale groupoid with a zero-dimensional orbit space. This is the case when $\liminf _{t \rightarrow \infty} t^{-1} \operatorname{diam}(X, h(t))=0$. The unit space of the groupoid is locally homogeneous with respect to the local action of the sheaf $\mathfrak{n}$ of nilpotent Lie algebras. The only possibilities for the stalk of $\mathfrak{n}$ are $\mathbb{R}^{3}$ or nil.

In the $\mathbb{R}^{3}$ case, the limiting Einstein flow must be a Kasner solution. In the nil case, the limiting Einstein flow must be the Taub-nil solution. Hence we can say that if $\lim _{t \rightarrow \infty} t^{-1} \operatorname{diam}(X, h(t))=0$ then at large times, after rescaling the geometry is modeled by one of these two solutions.

The rescalings of a Taub-nil solution approach a Kasner solution. Using this fact, if $\lim \inf _{t \rightarrow \infty} t^{-1} \operatorname{diam}(X, h(t))=0$ then we show that there is a sequence of times going to infinity so that after rescaling, the geometry is modeled by a Kasner solution.

We first recall some facts about homogeneous Einstein solutions from [15]. The only $\mathbb{R}^{3}$-invariant expanding CMC Einstein flows on $\mathbb{R}^{3}$ (up to time translation) are the Kasner solutions

$$
g=-d u^{2}+u^{2 p_{1}} d x^{2}+u^{2 p_{2}} d y^{2}+u^{2 p_{3}} d z^{2},
$$

where $p_{1}+p_{2}+p_{3}=p_{1}^{2}+p_{2}^{2}+p_{3}^{2}=1$. Equation (3.11) is not in CMC form. Putting it in $\mathrm{CMC}$ form and using the time parameter $-\frac{3}{H}$ removes the time translation freedom.

The only left-invariant expanding CMC Einstein flows on Nil (up to time translation) are the Taub-nil solutions

$$
g=-A^{2} d u^{2}+u^{2 p_{1}} A^{-2}\left(d x+4 p_{1} b z d y\right)^{2}+u^{2 p_{2}} A^{2} d y^{2}+u^{2 p_{3}} A^{2} d z^{2},
$$

where $A^{2}=1+b^{2} u^{4 p_{1}}$ and $p_{1}+p_{2}+p_{3}=p_{1}^{2}+p_{2}^{2}+p_{3}^{2}=1$. Equation (3.12) is not in $\mathrm{CMC}$ form. Putting it in CMC form and using the time parameter $-\frac{3}{H}$ removes the time translation freedom.

With reference to Corollary [3.4, suppose that the groupoid $\mathcal{X}^{\infty} \operatorname{has} \operatorname{dim}(\mathfrak{n})=3$. Then the orbit space is a point.

Let $\mathcal{E}$ be an Einstein flow on a 3 -dimensional étale groupoid $\mathcal{X}$ with $\operatorname{dim}(\mathfrak{n})=3$. Then the stalk of $\mathfrak{n}$ is $\mathbb{R}^{3}$ or nil. If the stalk is $\mathbb{R}^{3}$ then there is a cross-product description $\mathcal{X}=\mathbb{R}^{3} \rtimes \Gamma$, where $\Gamma$ is a group (with the discrete topology) that contains $\mathbb{R}^{3}$ as a finiteindex subgroup of translations. We say that $\mathcal{E}$ is of Kasner type. 
If the stalk of $\mathfrak{n}$ is $n i l$ then $\mathcal{X}=N i l \rtimes \Gamma$, where $\Gamma$ is a group (with the discrete topology) that contains $N i l$ as a finite-index subgroup, acting by left multiplication. We say that $\mathcal{E}$ is of Taub-nil type. One can check as $s \rightarrow \infty$, the rescaled Einstein flow $\mathcal{E}_{s}^{\infty}$ approaches an Einstein flow of Kasner type (3.11) with the same indices $\left(p_{1}, p_{2}, p_{3}\right)$.

Let Kas denote the Einstein flows of Kasner type on 3-dimensional étale groupoids whose orbit space is a point. Let Taub-nil denote the Einstein flows of Taub-nil type on 3-dimensional étale groupoids whose orbit space is a point.

Proposition 3.13. Let $\mathcal{E}$ be a type-III Einstein flow on a compact manifold $X$. Suppose that there is a sequence $\left\{t_{i}\right\}_{i=1}^{\infty}$ with $\lim _{i \rightarrow \infty} t_{i}=\infty$ so that $\lim _{i \rightarrow \infty} t_{i}^{-1} \operatorname{diam}\left(X, h\left(t_{i}\right)\right)=0$. Then $X$ has Thurston type $\mathbb{R}^{3}$ or Nil. As $i \rightarrow \infty$,

(1) If $X$ has Thurston type $\mathbb{R}^{3}$ then the rescaled Einstein flow $\mathcal{E}_{t_{i}}$ approaches Kas in the weak $W^{2, p}$-topology and the $C^{1, \alpha}$-topology.

(2) If $X$ has Thurston type $N i$ then the rescaled Einstein flow $\mathcal{E}_{t_{i}}$ approaches $K a s \cup$ Taub-nil in the weak $W^{2, p}$-topology and the $C^{1, \alpha}$-topology.

Proof. Since $X$ admits a sequence of Riemannian metrics $\left\{h\left(t_{i}\right)\right\}_{i=1}^{\infty}$ with $\lim _{i \rightarrow \infty}|\operatorname{Rm}|_{h\left(t_{i}\right)} \operatorname{diam}^{2}\left(X, h\left(t_{i}\right)\right)=$ 0 , it is an almost flat manifold and hence of Thurston type $\mathbb{R}^{3}$ or $N i l$. Suppose that $X$ has Thurston type $\mathbb{R}^{3}$. Consider any subsequence of the $t_{i}$ 's, which we relabel as $\left\{t_{i}\right\}_{i=1}^{\infty}$. From Corollary [3.4, a further subsequence converges to an Einstein flow $\mathcal{E}^{\infty}$ on an étale groupoid $\mathcal{X}^{\infty}$, with a zero dimensional orbit space from the diameter bound. Since $X$ is a finite quotient of $T^{3}$, the local symmetry algebra $\mathfrak{n}^{\infty}$ of $\mathcal{X}^{\infty}$ must be $\mathbb{R}^{3}$. Hence $\mathcal{E}^{\infty} \in$ Kas.

If $X$ has Thurston type $N i l$ then we can again construct $\mathcal{E}^{\infty}$. Now the stalk of $\mathfrak{n}^{\infty}$ is $\mathbb{R}^{3}$ or nil. Hence $\mathcal{E}^{\infty} \in$ Kas $\cup$ Taub-nil.

Corollary 3.14. Under the hypotheses of Proposition 3.13 , let $\widetilde{\mathcal{E}}$ denote the pullback Einstein flow on the universal cover $\widetilde{X}$. Choose $\widetilde{x}_{i} \in \tilde{X}$. Under conclusion (1) of Proposition 3.13, $\lim _{i \rightarrow \infty} \widetilde{\mathcal{E}}_{t_{i}}$ approaches the set of pointed Kasner solutions on $\mathbb{R}^{3}$, in the pointed weak $W^{2, p}$-topology and the pointed $C^{1, \alpha}$-topology. Under conclusion (2) of Proposition [3.13, $\left\{\widetilde{\mathcal{E}}_{t_{i}}, \widetilde{x}_{i}\right\}_{i=1}^{\infty}$ approaches the set of pointed Kasner and Taub-nil solutions on $\mathbb{R}^{3}$, in the pointed weak $W^{2, p}$-topology and the pointed $C^{1, \alpha}$-topology.

Proof. Given Proposition 3.13, the corollary follows as in [22, Section 6.2].

Corollary 3.15. Under the hypotheses of Proposition 3.13, there is a sequence $\left\{t_{j}^{\prime}\right\}_{i=1}^{\infty}$ with $\lim _{j \rightarrow \infty} t_{j}^{\prime}=\infty$ so that the rescalings $\left\{\mathcal{E}_{t_{j}^{\prime}}\right\}_{j=1}^{\infty}$ approach an Einstein flow of Kasner type on a three dimensional étale groupoid, in the weak $W^{2, p}$-topology and the $C^{1, \alpha}$-topology.

Proof. Proposition 3.13 implies that after passing to a subsequence of $\left\{t_{i}\right\}_{i=1}^{\infty}$, which we relabel as $\left\{t_{i}\right\}_{i=1}^{\infty}$, there is a $\operatorname{limit}_{\lim _{i \rightarrow \infty}} \mathcal{E}_{t_{i}}=\mathcal{E}^{\infty}$, with the stalk of $\mathfrak{n}^{\infty}$ equal to $\mathbb{R}^{3}$ or nil. If the stalk is $\mathbb{R}^{3}$ then $\mathcal{E}^{\infty} \in$ Kas and we can take $t_{i}^{\prime}=t_{i}$. Suppose that the stalk is nil. Then $\mathcal{E}^{\infty} \in$ Taub - nil. As $\lim _{s \rightarrow \infty} \mathcal{E}_{s}^{\infty}=\mathcal{E}^{\infty, \infty}$ for some $\mathcal{E}^{\infty, \infty} \in$ Kas, we can find a sequence $\left\{s_{j}\right\}_{i=1}^{\infty}$ with $\lim _{j \rightarrow \infty} s_{j}=\infty$ so that $\lim _{j \rightarrow \infty} \mathcal{E}_{s_{j}}^{\infty}=\mathcal{E}^{\infty, \infty}$. From the definition of convergence of flows, we can find a subsequence $\left\{t_{i_{j}}\right\}_{j=1}^{\infty}$ of $\left\{t_{i}\right\}_{i=1}^{\infty}$ so that $\lim _{j \rightarrow \infty} \mathcal{E}_{s_{j} t_{i}}=\mathcal{E}^{\infty, \infty}$. Putting $t_{j}^{\prime}=s_{j} t_{i_{j}}$, the corollary follows. 
Let $\tilde{X}$ denote the universal cover of $X$. We give it the pullback Einstein flow.

Corollary 3.16. Under the hypotheses of Corollary 3.15 , choose $\widetilde{x}_{j}^{\prime} \in \widetilde{X}$. Then $\left\{\widetilde{\mathcal{E}}_{t_{j}^{\prime}}, \widetilde{x}_{j}^{\prime}\right\}_{j=1}^{\infty}$ approaches the set of pointed Kasner solutions on $\mathbb{R}^{3}$, in the pointed weak $W^{2, p}$-topology and the pointed $C^{1, \alpha}$-topology.

Proof. Given Corollary 3.15, the corollary follows as in [22, Section 6.2].

Remark 3.17. Under the hypotheses of Proposition 3.13, it does not immediately follow that as $i \rightarrow \infty$, the rescaled Einstein flows $\left\{\mathcal{E}_{t_{i}}\right\}_{i=1}^{\infty}$ approach an Einstein flow of Kasner type. For example, it is conceivable that there is an infinite number of increasingly sparse subsequences $\left\{t_{j, m}\right\}_{j=1}^{\infty}$ so that for each $m$, the $\operatorname{limit}_{\lim _{j \rightarrow \infty}} \mathcal{E}_{t_{j, m}}$ exists and is always the same Taub-nil solution. We do not know if there is an example where the rescaled flows $\left\{\mathcal{E}_{t_{i}}\right\}_{i=1}^{\infty}$ approach a Taub-nil solution.

3.3. One dimensional orbit space. In this subsection we deal with the case when a limiting Einstein flow $\mathcal{E}^{\infty}$ is on an étale groupoid whose orbit space is one dimensional, i.e. is a circle or an interval. The goal is to show that after performing a further rescaling, there is a new limit $\mathcal{E}^{\infty, \infty}$ which is a Taub-flat flow; hence an appropriate rescaling limit of the original Einstein flow $\mathcal{E}$ is a Taub-flat flow.

If $\mathcal{E}$ has $\lim \inf _{t \rightarrow \infty} t^{-1} \operatorname{diam}(X, h(t))=0$ then we can consider the flow to be treated by Corollary 3.15. Hence we assume that $\operatorname{diam}(X, h t)) \geq c t$ for all $t \in\left[t_{0}, \infty\right)$ and some $c>0$. Then the limiting flow $\mathcal{E}^{\infty}$ satisfies $\operatorname{diam}\left(\mathcal{X}^{\infty}, h^{\infty}(u)\right) \geq c u$ for all $u>0$. This means that any rescaling limit $\mathcal{E}^{\infty, \infty}$ of $\mathcal{E}^{\infty}$ also has a one dimensional orbit space.

Using the type-III assumption on the original flow $\mathcal{E}$, we argue that a rescaling limit $\mathcal{E}^{\infty, \infty}$ of $\mathcal{E}^{\infty}$ exists. Then the issue is to show that it is a Taub-flat flow. In order to do this, we need a monotonic quantity. The Einstein flow $\mathcal{E}^{\infty}$ has $\operatorname{dim}(\mathfrak{n})=2$, i.e. has local $\mathbb{R}^{2}$-symmetries. The metric in the $\mathbb{R}^{2}$-directions is locally given by a $2 \times 2$ matrix $G$, whose determinant is a well-defined function on the two dimensional Lorentzian manifold $(0, \infty) \times X^{\infty}$. We can assume that $\mathcal{E}^{\infty}$ is not already a Taub-flat flow. Then it is known that $\nabla \operatorname{det} G$ is a nonvanishing timelike vector on $(0, \infty) \times X^{\infty}$. The level sets of $\operatorname{det} G$ are spacelike submanifolds; we assume that they are compact. Then we can use the monotonic quantities defined in Subsection A.3.

We obtain an integral convergence result along the lines of Proposition 2.36. To go further, we make the additional assumption that there is a time function $\widehat{u}$ for the foliation of $(0, \infty) \times X^{\infty}$ by level sets of $\operatorname{det}(G)$, which is comparable to $u$. Using this time function and the monotonic quantities from Subsection A.3, we deduce that $\mathcal{E}^{\infty, \infty}$ is a Taub-flat flow. Hence there are arbitrarily large future time intervals on which the original flow $\mathcal{E}$ is modelled, in a scale invariant way, by a Taub-flat flow.

To begin, the Taub-flat vacuum solution is the isometric product of $\mathbb{R}^{2}$ with the Lorentzian cone over $H^{1} \cong \mathbb{R}^{1}$. Suppose that $\mathcal{X}$ is a three dimensional cross-product groupoid $\left(H^{1} \times \mathbb{R}^{2}\right) \rtimes \Gamma$, where $\Gamma$ is a group (with the discrete topology) that contains the translations $L \mathbb{Z} \times \mathbb{R}^{2}$ as a finite-index subgroup, for some $L>0$. We say that an Einstein flow on $\mathcal{X}$ is of Taub-flat type if the corresponding Lorentzian groupoid is equivalent to the cross product of $\Gamma$ with the Taub-flat solution. 
Let $\mathcal{E}$ be an Einstein flow as in the hypotheses of Corollary 3.4, satisfying Assumption 3.10. If $X$ has Thurston type $S o l$ and $\left\{t_{i}\right\}_{i=1}^{\infty}$ is a sequence with $\lim _{i \rightarrow \infty} t_{i}=\infty$ then a Gromov-Hausdorff limit of $\left\{\left(X, t_{i}^{-2} h\left(t_{i}\right)\right)\right\}_{i=1}^{\infty}$ cannot be three dimensional by Proposition 2.56. It cannot be zero dimensional (or else $X$ would have Thurston type $\mathbb{R}^{3}$ or $N i l$ ) and it cannot be two dimensional (or else $X$ would be a Seifert 3-manifold). Thus if $X$ has Thurston type $S o l$ then a Gromov-Hausdorff limit of $\left\{\left(X, t_{i}^{-2} h\left(t_{i}\right)\right)\right\}_{i=1}^{\infty}$ must be one dimensional. More generally, if $\left\{\left(X, t_{i}^{-2} h\left(t_{i}\right)\right)\right\}_{i=1}^{\infty}$ has a one dimensional Gromov-Hausdorff limit then $X$ must have Thurston type $S o l, \mathbb{R}^{3}$ or $N i l$.

With reference to Corollary 3.4, suppose that the groupoid $\mathcal{X}^{\infty} \operatorname{has} \operatorname{dim}(\mathfrak{n})=2$. Then the orbit space is one dimensional. If $X$ has Thurston type $S o l$ then there is some $c>0$ so that $\operatorname{diam}(X, h(t)) \geq c t$ for all $t \geq t_{0}$, as $X$ is not almost flat. If $X$ has Thurston type $\mathbb{R}^{3}$ or $N i l$, and $\liminf _{t \rightarrow \infty} t^{-1} \operatorname{diam}(X, h(t))=0$, then we can consider the Einstein flow to be covered by Corollary 3.15. Hence we make the following assumption.

Assumption 3.18. For some $c>0$, we have $\operatorname{diam}(X, h(t)) \geq$ ct for all $t \in\left[t_{0}, \infty\right)$.

With reference to Corollary [3.4, letting $X^{\infty}$ denote the orbit space of $\mathcal{X}^{\infty}$, we loosely write $\operatorname{diam}\left(\mathcal{X}^{\infty}, h^{\infty}(u)\right)$ for the diameter of $X^{\infty}$ with the induced metric. Then

$$
\operatorname{diam}\left(\mathcal{X}^{\infty}, h^{\infty}(u)\right) \geq c u
$$

for all $u \in(0, \infty)$.

The orbit space $X^{\infty}$ is a one dimensional orbifold [22, Pf. of Proposition 3.5]. Hence it is $S^{1}$ or $S^{1} / \mathbb{Z}_{2}$. In the latter case, we can pullback under the orbifold covering map $S^{1} \rightarrow S^{1} / \mathbb{Z}_{2}$ to reduce the statements to the $S^{1}$ case. Hence we assume that the orbit space $X^{\infty}$ is diffeomorphic to $S^{1}$.

The coordinate function $u$ on $(0, \infty) \times \mathcal{X}^{\infty}$ pulls back from a function on $(0, \infty) \times S^{1}$, which we again denote by $u$. Similarly, the lapse function $L$ pulls back from a function on $(0, \infty) \times S^{1}$, which we again denote by $L$. As in Subsection A.1, the Lorentzian metric corresponding to the Einstein flow $\mathcal{E}^{\infty}$ can locally be written as

$$
-L^{2} d u^{2}+h d \theta^{2}+\sum_{I, J=1}^{2} G_{I J}\left(d b^{I}+A^{I}\right)\left(d b^{J}+A^{J}\right) .
$$

We note that $\operatorname{det}(G)$ is a well defined function on $(0, \infty) \times S^{1}$, since the flat twisting bundle $e$ on $(0, \infty) \times S^{1}$ has holonomy in $\operatorname{SL}(2, \mathbb{Z})\left[22\right.$, Proof of Lemma 6.1]. Put $g=-L^{2} d u^{2}+h d \theta^{2}$.

If $\mathcal{E}^{\infty}$ is not flat then the function $\operatorname{det}(G)$ has a timelike gradient on $(0, \infty) \times S^{1}$; see [20, Proof of Proposition 5.1] and references therein. If $\mathcal{E}^{\infty}$ is flat then the results of this subsection will be valid, so we assume that $\mathcal{E}^{\infty}$ is not flat. Then $\operatorname{det}(G)$ has level sets that foliate $(0, \infty) \times S^{1}$. We assume that $\nabla \operatorname{det}(G)$ is future-directed; this holds, for example, in the Ellis-MacCallum Sol-solution [15, Section 9.2.3]. Then we can choose a time function $\widehat{u}$ on $(0, \infty) \times S^{1}$ that is an increasing function of $\operatorname{det}(G)$.

Assumption 3.21. There is an open set $U \subset(0, \infty) \times S^{1}$ containing $\left[u_{0}, \infty\right) \times S^{1}$ for some $u_{0}<\infty$, and a proper function $\widehat{u} \in W_{\text {loc }}^{3, p}(U)$ so that

(1) $\nabla \widehat{u}$ is timelike, and 
(2) On $U$, $\operatorname{det}(G)$ is a function of $\widehat{u}$.

Assumption 3.21 implies the level sets of $\widehat{u}$ are compact manifolds. We can assume that they are diffeomorphic to $S^{1}$. Then for suitable $\widehat{u}_{0}<\infty$, the space $\widehat{u}^{-1}\left(\left[\widehat{u}_{0}, \infty\right)\right)$ is $W_{l o c}^{3, p}$ diffeomorphic to $\left[\widehat{u}_{0}, \infty\right) \times S^{1}$. We write the Lorentzian metric $g$ on $\left[\widehat{u}_{0}, \infty\right) \times S^{1}$, in terms of $\widehat{u}$ and $\theta$, as $\widehat{g}=-\widehat{L}^{2} d \widehat{u}^{2}+\widehat{h} d \theta^{2}$.

If the curvature $F$ of the $\mathbb{R}^{N}$-valued connection $A$ vanishes then from (A.20),

$$
\int_{\widehat{u}_{0}}^{\infty} \frac{1}{\sqrt{\operatorname{det} G}} \widehat{L}^{-1} \operatorname{Tr}\left(\left(G^{-1} \partial_{\widehat{u}} G\right)^{2}\right) \operatorname{dvol}\left(S^{1}, \widehat{h}(\widehat{u})\right) d \widehat{u}<\infty .
$$

Given $s>0$, define $\widehat{L}_{s}$ and $\widehat{h}_{s}$ as in (2.35). Put $G_{s}(\widehat{v})=G(s \widehat{v})$.

Proposition 3.23. Suppose that $F=0$. Given $\Lambda>1$, we have

$$
\lim _{s \rightarrow \infty} \frac{1}{\sqrt{\operatorname{det} G_{s}}} \widehat{L}_{s}^{-1} \operatorname{Tr}\left(\left(G_{s}^{-1} \partial_{\widehat{v}} G_{s}\right)^{2}\right) d \widehat{v} \operatorname{dvol}\left(S^{1}, \widehat{h}_{s}(\widehat{v})\right)=0
$$

in norm convergence of measures on $\left[\Lambda^{-1}, \Lambda\right] \times S^{1}$.

Proof. The proof is similar to that of Proposition 2.36. We omit the details.

Remark 3.25. From Subsection A.3, if $G_{s}^{-1} \partial_{\widehat{v}} G_{s}=0$ then $G$ is locally constant in $\widehat{v}$ and $\theta$, and $\widehat{g}$ is flat. Hence Proposition 3.23 can be interpreted as saying that in an integral sense, the original flow $\mathcal{E}$ is approaching a flow of Taub-flat type. If $F \neq 0$ then there is a result analogous to Proposition 3.23, except more complicated to state, using (A.27) and (A.28).

We now make a further assumption about $\widehat{u}$, saying that it is comparable to $u$.

Assumption 3.26. In addition to Assumption 3.21, suppose that there is a constant $\Lambda<$ $\infty$ so that

(1) $\Lambda^{-1} u \leq \widehat{u} \leq \Lambda u$,

(2) For all $r>u_{0}, p<\infty$ and $k+l \leq 3$,

$$
\left\|\nabla_{x}^{k} \partial_{u}^{l} \widehat{u}\right\|_{L^{p}\left((r, 2 r) \times X^{\infty}\right)} \leq \text { const. } r^{1-k-l+\frac{2}{p}} \text { and }
$$

(3) $\frac{g(\nabla u, \nabla \widehat{u})}{|\nabla u|_{g}|\nabla \widehat{u}|_{g}} \leq-\Lambda^{-1}$.

Remark 3.28. The exponent on the right-hand side of (3.27) ensures scale invariance.

Proposition 3.29. If Assumption [3.26 holds then there is a sequence $\left\{t_{j}^{\prime}\right\}_{j=1}^{\infty}$ with $\lim _{j \rightarrow \infty} t_{j}^{\prime}=$ $\infty$, and an Einstein flow $\mathcal{E}^{\infty, \infty}$ of Taub-flat type, so that the rescalings $\mathcal{E}_{t_{j}^{\prime}}$ of $\mathcal{E}$ satisfy $\lim _{j \rightarrow \infty} \mathcal{E}_{t_{j}^{\prime}}=\mathcal{E}^{\infty, \infty}$.

Proof. Let $\left\{s_{j}\right\}_{j=1}^{\infty}$ be a sequence with $\lim _{j \rightarrow \infty} s_{j}=\infty$. Since $\lim _{i \rightarrow \infty} \mathcal{E}_{t_{i}}=\mathcal{E}^{\infty}$, for fixed $j$, we have $\lim _{i \rightarrow \infty} \mathcal{E}_{s_{j} t_{i}}=\mathcal{E}_{s_{j}}^{\infty}$. If $\left\{t_{i_{j}}\right\}_{j=1}^{\infty}$ is a subsequence of $\left\{t_{i}\right\}_{i=1}^{\infty}$ then after passing to a subsequence of $j$ 's, we can assume that $\lim _{j \rightarrow \infty} \mathcal{E}_{s_{j} t_{i}}=\mathcal{E}^{\infty, \infty}$ for an Einstein flow $\mathcal{E}^{\infty, \infty}$ on an étale groupoid $\mathcal{X}^{\infty, \infty}$, defined on the time interval $(0, \infty)$. From our definition of convergence of flows, we can choose $\left\{t_{i_{j}}\right\}_{j=1}^{\infty}$ so that $\lim _{j \rightarrow \infty} \mathcal{E}_{s_{j}}^{\infty}=\lim _{j \rightarrow \infty} \mathcal{E}_{s_{j} t_{i j}}=$ $\mathcal{E}^{\infty, \infty}$. (The rescaling in $\mathcal{E}_{s_{j}}^{\infty}$ involves pullback with respect to $u \rightarrow s_{j} u$ and a $j$-dependent 
diffeomorphism $\phi_{j}$ of $S^{1}$, along with a $j$-dependent automorphism of the flat $\mathbb{R}^{2}$-vector bundle on $S^{1}$.) From Assumption 3.18, the orbit space of $\mathcal{X}^{\infty, \infty}$ is one dimensional. The Lorentzian metric corresponding to the Einstein flow $\mathcal{E}^{\infty, \infty}$ can be locally written as

$$
-\left(L^{\infty}\right)^{2}\left(d u^{\infty}\right)^{2}+h^{\infty}\left(d \theta^{\infty}\right)^{2}+\sum_{I, J=1}^{2} G_{I J}^{\infty}\left(d b^{I}+A^{\infty, I}\right)\left(d b^{J}+A^{\infty, J}\right) .
$$

We will show this is an Einstein flow of Taub-flat type and take $t_{j}^{\prime}=s_{j} t_{i_{j}}$.

Let $\widehat{X}^{\infty}$ denote the level sets of $\widehat{u}$. Let $\widehat{u}_{s_{j}}$ be $\frac{1}{s_{j}}$ times the pullback of $\widehat{u}$ with respect to $u \rightarrow s_{j} u$ and $\phi_{j} \in \operatorname{Diff}\left(X^{\infty}\right)$. From Assumption 3.26 $(1,2)$, after passing to a subsequence we can assume that $\lim _{j \rightarrow \infty} \widehat{u}_{s_{j}}=\widehat{u}^{\infty}$ in the weak topology on $W_{\text {loc }}^{3, p}$, for some $\widehat{u}^{\infty} \in$ $W_{l o c}^{3, p}\left((0, \infty) \times X^{\infty, \infty}\right)$. From Assumption 3.21 and Assumption $3.26(3)$, the gradient $\nabla \widehat{u}^{\infty}$ is timelike, and $\operatorname{det}(G)$ is a function of $\widehat{u}^{\infty}$. We can write the Lorentian metric on $(0, \infty) \times$ $X^{\infty, \infty}$ as

$$
-\left(\widehat{L}^{\infty}\right)^{2}\left(d \widehat{u}^{\infty}\right)^{2}+\widehat{h}^{\infty}\left(d \widehat{\theta}^{\infty}\right)^{2} .
$$

Suppose first that the curvature $F^{\infty}$ of $A^{\infty}$ vanishes. Applying Subsection A.2 to the flow $\mathcal{E}^{\infty}$, we know that $\left(\partial_{\widehat{u}} \ln \operatorname{det} G\right) \int_{\widehat{X}^{\infty}} \widehat{L}^{-1} \mathrm{dvol}_{\widehat{X}^{\infty}}$ is monotonically nonincreasing in $\widehat{u}$. It is clearly nonnegative. Since $\operatorname{dim}\left(\widehat{X}^{\infty}\right)=1$, the expression is invariant under rescaling. Note that in forming the $\operatorname{limit}_{\lim _{j \rightarrow \infty}} \mathcal{E}_{s_{j}}^{\infty}=\mathcal{E}^{\infty, \infty}$, we are allowed to perform $j$-dependent automorphisms of the flat 2-dimensional vector bundle on $X^{\infty}$. These automorphisms can change $\ln \operatorname{det} G$ by a $j$-dependent additive constant, which vanishes upon taking the $\widehat{u}$-derivative.

Given $a \in(0, \infty)$, the level set $\left(\widehat{u}^{\infty}\right)^{-1}(a) \subset(0, \infty) \times X^{\infty, \infty}$ is the limit of rescalings of level sets $\widehat{u}^{-1}\left(s_{j} a\right) \subset(0, \infty) \times X^{\infty}$. It follows that the monotonic quantity $\left(\partial_{\widehat{u}^{\infty}} \ln \operatorname{det} G^{\infty}\right) \int_{\widehat{X}^{\infty, \infty}}\left(\widehat{L}^{\infty}\right)^{-1} \operatorname{dvol}_{\widehat{X}^{\infty, \infty}}$ is constant in $\widehat{u}^{\infty}$. By Subsubsection A.3.1, we conclude that $\mathcal{E}^{\infty, \infty}$ is a flat solution.

Remark 3.32. We could have reached the same conclusion using the functional $\widehat{\mathcal{E}}$ of (A.19).

Now suppose that $F^{\infty} \neq 0$. After pulling back from a finite cover of $S^{1}$ if necessary, we can assume that the holonomy $H \in \mathrm{SL}(2, \mathbb{R})$ over $S^{1}$ of the flat vector bundle $E$ has real positive eigenvalues. The functional $\widehat{\mathcal{E}}_{K}$ of $(\mathrm{A} .26)$ is scale invariant and monotonically nonincreasing. It follows that the corresponding functional for $\mathcal{E}^{\infty, \infty}$ is constant. In terms of Subsubsection A.3.2, the metric (3.30) equals (A.29), after a change of variable from $u^{\infty}$ to $R$. From Corollary 3.4, the function $L^{\infty}$ is uniformly bounded below and so the metric (3.30) admits future-directed timelike curves along which the proper time goes to infinity. Hence under the change of variable from $u^{\infty}$ to $R$, we must have $\lim _{u^{\infty} \rightarrow \infty} R\left(u^{\infty}\right)=\infty$. From (A.29), the length of the $S^{1}$-fiber is uniformly bounded as $R$ goes to infinity. This contradicts (3.19), showing that $F^{\infty}$ cannot be nonzero.

Hence $\mathcal{E}^{\infty, \infty}$ is a flat solution. There is a foliation of $(0, \infty) \times X^{\infty, \infty}$ by circles $\left\{C_{v}\right\}_{v \in(0, \infty)}$ of constant geodesic curvature $-\frac{1}{v}$. The lift $\widetilde{C}_{v}$ of such a circle to the universal cover $(0, \infty) \times$ $\widetilde{X}^{\infty, \infty}$ is an embedded curve with a neighborhood that is isometric to a neighborhood of 
a hyperbola, of constant geodesic curvature $-\frac{1}{v}$, in the flat Lorentzian plane $\mathbb{R}^{1,1}$. As $(0, \infty) \times \widetilde{X}^{\infty, \infty}$ is foliated by such lifts, it must be isometric to the chronological future of the origin in $\mathbb{R}^{1,1}$, with its foliation by hyperbolas. Then $(0, \infty) \times X^{\infty, \infty}$ is the Lorentzian cone over a circle, and $\mathcal{E}^{\infty, \infty}$ is an Einstein flow of Taub-flat type.

Let $\widetilde{X}$ denote the universal cover of $X$. We give it the pullback Einstein flow.

Corollary 3.33. Under the hypotheses of Proposition 3.29 , choose $\widetilde{x}_{j}^{\prime} \in \widetilde{X}$. Then $\left\{\left(\widetilde{\mathcal{E}}_{t_{j}^{\prime}}, \widetilde{x}_{j}^{\prime}\right)\right\}_{j=1}^{\infty}$ approaches the set of Taub-flat Einstein flows on $\mathbb{R}^{3}$, in the pointed weak $W^{2, p}$-topology and the pointed $C^{1, \alpha}$-topology.

Proof. Given Proposition 3.29, the corollary follows as in [22, Section 6.2].

3.4. Two dimensional orbit space. In this subsection we deal with the case when a limiting Einstein flow $\mathcal{E}^{\infty}$ is on an étale groupoid whose orbit space is two dimensional. In our case, it will necessarily be a two dimensional orbifold. The goal is to show that after performing a further rescaling, there is a new limit $\mathcal{E}^{\infty, \infty}$ which is a Bianchi-III flat flow; hence an appropriate rescaling limit of the original Einstein flow $\mathcal{E}$ is a Bianchi-III flat flow.

If $\mathcal{E}$ has a rescaling limit whose orbit space has dimension zero or one then we can consider the flow to be treated by Corollary 3.15 and Proposition 3.29. Hence we assume that there is no such rescaling limit. This implies that any rescaling limit $\mathcal{E}^{\infty, \infty}$ of $\mathcal{E}^{\infty}$ also has a two dimensional orbit space.

Using the type-III assumption on the original flow $\mathcal{E}$, we argue that a rescaling limit $\mathcal{E}^{\infty, \infty}$ of $\mathcal{E}^{\infty}$ exists. Then the issue is to show that it is a Bianchi-III flat flow. In order to do this, we make the conformal change of Subsection A.4 and assume that the ensuing Lorentzian 3-manifold has an expanding CMC foliation, Using the monotonic quantity of Subsection A.4, we obtain an integral convergence result along the lines of Proposition 2.36. To go further, we make the additional assumption that there is a time function $\widehat{u}$ for the new CMC foliation, which is comparable to $u$. Using this time function and the monotonic quantity from Subsection A.4, we deduce that $\mathcal{E}^{\infty, \infty}$ is a Bianchi-III flat flow. Hence there are arbitrarily large future time intervals on which the original flow $\mathcal{E}$ is modelled, in a scale invariant way, by a Bianchi-III flat flow.

To begin, the Bianchi-III flat vacuum solution is the isometric product of $\mathbb{R}$ with the Lorentzian cone over $H^{2}$. Suppose that $\mathcal{X}$ is a three dimensional cross-product groupoid $\left(\mathbb{R} \times \mathbb{R}^{2}\right) \rtimes \Gamma$, where $\Gamma$ is a group (with the discrete topology) that contains $\mathbb{R} \times \Gamma_{0}$ as a finite-index subgroup, with $\Gamma_{0}$ being a discrete subgroup of $\operatorname{Isom}\left(H^{2}\right)$. We say that an Einstein flow on $\mathcal{X}$ is of Bianchi-III flat type if the corresponding Lorentzian groupoid is the cross-product of $\Gamma$ with the Bianchi-III flat vacuum solution.

Let $\mathcal{E}$ be an Einstein flow as in the hypotheses of Corollary 3.4, satisfying Assumption 3.10. If $X$ has Thurston type $H^{2} \times \mathbb{R}$ or $\mathrm{SL}(2, \mathbb{R})$, and $\left\{t_{i}\right\}_{i=1}^{\infty}$ is a sequence with $\lim _{i \rightarrow \infty} t_{i}=$ $\infty$, then a Gromov-Hausdorff limit of $\left\{\left(X, t_{i}^{-2} h\left(t_{i}\right)\right)\right\}_{i=1}^{\infty}$ cannot be three dimensional by Proposition 2.56. It cannot be zero dimensional (or else $X$ would have Thurston type $\mathbb{R}^{3}$ or $N i l$ ) and it cannot be one dimensional (or else a finite cover of $X$ would be the total 
space of a $T^{2}$-bundle over a circle). Thus if $X$ has Thurston type $H^{2} \times \mathbb{R}$ or $\widetilde{\mathrm{SL}(2, \mathbb{R})}$ then a Gromov-Hausdorff limit of $\left\{\left(X, t_{i}^{-2} h\left(t_{i}\right)\right)\right\}_{i=1}^{\infty}$ must be two dimensional. More generally, if there is a sequence $\left\{t_{i}\right\}_{i=1}^{\infty}$ with $\lim _{i \rightarrow \infty} t_{i}=\infty$ so that $\left\{\left(X, t_{i}^{-2} h\left(t_{i}\right)\right)\right\}_{i=1}^{\infty}$ has a two

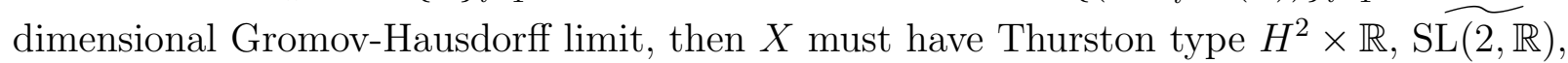
$\mathbb{R}^{3}$ or Nil.

With reference to Corollary [3.4, suppose that the groupoid $\mathcal{X}^{\infty} \operatorname{has} \operatorname{dim}(\mathfrak{n})=1$. Then the orbit space is two dimensional.

If there is a sequence $\left\{t_{i}\right\}_{i=1}^{\infty}$ with $\lim _{i \rightarrow \infty} t_{i}=\infty$ so that $\left\{\left(X, t_{i}^{-2} h\left(t_{i}\right)\right)\right\}_{i=1}^{\infty}$ has a GromovHausdorff limit of dimension less than two, then we can consider the Einstein flow to be covered by Corollary 3.15 and Proposition 3.29 (modulo the verification of Assumption 3.26). Hence we make the following assumption.

Assumption 3.34. There is no sequence $\left\{t_{i}\right\}_{i=1}^{\infty}$ with $\lim _{i \rightarrow \infty} t_{i}=\infty$ so that $\left\{\left(X, t_{i}^{-2} h\left(t_{i}\right)\right)\right\}_{i=1}^{\infty}$ has a Gromov-Hausdorff limit of dimension less than two.

Let $\mathcal{E}^{\infty}$ be a limit flow on an étale groupoid $\mathcal{X}^{\infty}$, as in Corollary 3.4. The orbit space $X^{\infty}$ of $\mathcal{X}^{\infty}$ is a two dimensional orbifold [22, Pf. of Proposition 3.5]. (This uses our assumption that $X$ is aspherical.) From Assumption 3.34, there is no subsequence $\left\{u_{j}\right\}_{j=1}^{\infty}$ with $\lim _{j \rightarrow \infty} u_{j}=\infty$ so that $\left\{\left(X^{\infty}, u_{j}^{-2} h^{\infty}\left(u_{j}\right)\right)\right\}_{j=1}^{\infty}$ has a Gromov-Hausdorff limit of dimension less than two.

The coordinate function $u$ on $(0, \infty) \times \mathcal{X}^{\infty}$ pulls back from a function on $(0, \infty) \times X^{\infty}$, which we again denote by $u$. Similarly, the lapse function $L$ pulls back from a function on $(0, \infty) \times X^{\infty}$, which we again denote by $L$. As in Subsection A.1, the Lorentzian metric corresponding to the groupoid Einstein flow $\mathcal{E}^{\infty}$ can locally be written as

$$
-L^{2} d u^{2}+\sum_{\alpha, \beta=1}^{2} h_{\alpha \beta} d b^{\alpha} d b^{\beta}+G(d \theta+A)^{2} .
$$

Put

$$
g=-L^{2} d u^{2}+\sum_{\alpha, \beta=1}^{2} h_{\alpha \beta} d b^{\alpha} d b^{\beta}
$$

$\widehat{g}=G g$ and $\widehat{G}=G^{2}$. Then

$$
g+G(d \theta+A)^{2}=\widehat{G}^{-\frac{1}{2}}\left(\widehat{g}+\widehat{G}(d \theta+A)^{2}\right) .
$$

Assumption 3.38. There is an open set $U \subset(0, \infty) \times X^{\infty}$ containing $\left[u_{0}, \infty\right) \times X^{\infty}$ for some $u_{0}<\infty$, and a proper function $\widehat{u} \in W_{\text {loc }}^{3, p}(U)$ so that

(1) $\nabla \widehat{u}$ is timelike, and

(2) On $U$, the level sets of $\widehat{u}$ have constant mean curvature with respect to $\widehat{g}$.

Assumption 3.38 implies the level sets of $\widehat{u}$ are compact. Let us denote their diffeomorphism type by $\widehat{X}^{\infty}$. Then for suitable $\widehat{u}_{0}<\infty$, the space $\widehat{u}^{-1}\left(\left[\widehat{u}_{0}, \infty\right)\right)$ is $W_{l o c}^{3, p}$-diffeomorphic to $\left[\widehat{u}_{0}, \infty\right) \times \widehat{X}^{\infty}$. 
Letting $\widehat{H}$ denote the (constant) mean curvatures of the level sets, suppose that $\widehat{H}$ is an increasing function in $\widehat{u}$ that takes all values in an interval $\left(-\widehat{H}_{0}, 0\right)$. Define a new time parameter by $v=-\frac{2}{\hat{H}}$. From (A.51),$v^{-2} \operatorname{dvol}\left(\widehat{X}^{\infty}, \widehat{h}(v)\right)$ is pointwise decreasing. Put

$$
\widehat{\operatorname{dvol}}_{\infty}=\lim _{v \rightarrow \infty} v^{-2} \operatorname{dvol}\left(\widehat{X}^{\infty}, \widehat{h}\right),
$$

an absolutely continuous measure on $\widehat{X}^{\infty}$. From (A.52),

$$
\begin{aligned}
& \frac{d}{d v}\left(v^{-2} \operatorname{vol}\left(\widehat{X}^{\infty}, \widehat{h}(v)\right)\right)=-v \int_{\widehat{X}^{\infty}}\left[\widehat{L}\left|\widehat{K}^{0}\right|^{2}+\frac{1}{4} \widehat{L}^{-1}\left|\widehat{S}_{0}\right|^{2}+\right. \\
& \left.\frac{1}{4} \widehat{L}^{-1}\left(\frac{\partial \ln \operatorname{det} \widehat{G}}{\partial v}\right)^{2}+\frac{1}{4} \widehat{L}^{i j} \widehat{h}^{k l} \widehat{G}_{I J} \widehat{F}_{i k}^{I} \widehat{F}_{j l}^{J}\right] \operatorname{dvol}\left(\widehat{X}^{\infty}, \widehat{h}(v)\right) .
\end{aligned}
$$

Given $s>0$, define $\widehat{L}_{s}, \widehat{h}_{s}, \widehat{K}_{s}$ and $\widehat{K}_{s}^{0}$ as in (2.35). Put $\widehat{G}_{s}(v)=\widehat{G}(s v)$ and $\widehat{F}_{s, i j}(v)=$ $s^{-1} \widehat{F}_{i j}(s v)$.

Proposition 3.41. Given $\Lambda>1$, we have

$$
\begin{aligned}
& \lim _{s \rightarrow \infty}\left(\widehat{L}_{s}-1\right)=\lim _{s \rightarrow \infty}\left|\widehat{K}^{0}\right|{ }_{s}^{2} \widehat{L}_{s}=\lim _{s \rightarrow \infty}\left|\widehat{S}^{0}\right|_{s}^{2} \widehat{L}_{s}=\lim _{s \rightarrow \infty}\left(\frac{\partial \ln \operatorname{det} \widehat{G}_{s}}{\partial v}\right)^{2} \widehat{L}_{s}^{-1}= \\
& \lim _{s \rightarrow \infty} \widehat{h}_{s}^{i j} \widehat{h}_{s}^{k l} \widehat{G}_{s, I J} \widehat{F}_{s, i k}^{I} \widehat{F}_{s, j l}^{J} \widehat{L}_{s}=0
\end{aligned}
$$

in $L^{1}\left(\left[\Lambda^{-1}, \Lambda\right] \times \widehat{X}_{\infty}, d v \widehat{\operatorname{dvol}}_{\infty}\right)$.

Proof. The proof is similar to that of Proposition 2.36, We omit the details.

Remark 3.43. From Subsection A.4, if $\widehat{L}-1=\widehat{K}^{0}=\widehat{S}^{0}=\frac{\partial \ln \operatorname{det} \widehat{G}}{\partial v}=\widehat{h}^{i j} \widehat{h}^{k l} \widehat{G}_{I J} \widehat{F}_{i k}^{I} \widehat{F}_{j l}^{J}=0$ then $\widehat{G}$ is locally constant and $\widehat{g}$ is flat. Hence Proposition 3.41 can be interpreted as saying that in an integral sense, the original flow $\mathcal{E}$ is approaching a flow of Bianchi-III flat type.

Define $\widehat{H}_{s, \Lambda}^{1}$ as in the paragraph before Proposition 2.41, replacing $h_{s}$ by $\widehat{h}_{s}$.

Proposition 3.44. We have

$$
\lim _{s \rightarrow \infty} d_{s y m m}\left(\widehat{H}_{s, \Lambda}^{1}, I_{n}\right)=0
$$

in $L^{2}\left(\widehat{X}_{\infty}, \widehat{\mathrm{dvol}_{\infty}}\right)$.

Proof. The proof is similar to that of Proposition 2.41. We omit the details.

We now make a further assumption about $\widehat{u}$, saying that it is comparable to $u$.

Assumption 3.46. In addition to Assumption 3.38, there is some $\Lambda<\infty$ so that

(1) $\Lambda^{-1} u \leq \widehat{u} \leq \Lambda u$, 
(2) For all $r>u_{0}, p<\infty$ and $k+l \leq 3$,

$$
\left\|\nabla_{x}^{k} \partial_{u}^{l} \widehat{u}\right\|_{L^{p}\left((r, 2 r) \times X^{\infty}\right)} \leq \text { const. } r^{1-k-l+\frac{3}{p}}
$$

(3) $\frac{a(\nabla u, \nabla \widehat{u})}{|\nabla u|_{g}|\nabla \widehat{u}|_{g}} \leq-\Lambda^{-1}$.

Remark 3.48. The exponent on the right-hand side of (3.47) ensures scale invariance.

Proposition 3.49. If Assumption 3.46 holds then there is a sequence $\left\{t_{j}^{\prime}\right\}_{j=1}^{\infty}$ with $\lim _{j \rightarrow \infty} t_{j}^{\prime}=$ $\infty$, and an Einstein flow $\mathcal{E}^{\infty, \infty}$ of Bianchi-III flat type, so that the rescalings $\mathcal{E}_{t_{j}^{\prime}}$ of $\mathcal{E}$ satisfy $\lim _{j \rightarrow \infty} \mathcal{E}_{t_{j}^{\prime}}=\mathcal{E}^{\infty, \infty}$.

Proof. Let $\left\{s_{j}\right\}_{j=1}^{\infty}$ be a sequence with $\lim _{j \rightarrow \infty} s_{j}=\infty$. Since $\lim _{i \rightarrow \infty} \mathcal{E}_{t_{i}}=\mathcal{E}^{\infty}$, for fixed $j$, we have $\lim _{i \rightarrow \infty} \mathcal{E}_{s_{j} t_{i}}=\mathcal{E}_{s_{j}}^{\infty}$. If $\left\{t_{i_{j}}\right\}_{j=1}^{\infty}$ is a subsequence of $\left\{t_{i}\right\}_{i=1}^{\infty}$ then after passing to a subsequence of $j$ 's, we can assume that $\lim _{j \rightarrow \infty} \mathcal{E}_{s_{j} t_{i}}=\mathcal{E}^{\infty, \infty}$ for an Einstein flow $\mathcal{E}^{\infty, \infty}$ on an étale groupoid $X^{\infty, \infty}$, defined on the time interval $(0, \infty)$. From our definition of convergence of flows, we can choose $\left\{t_{i_{j}}\right\}_{j=1}^{\infty}$ so that $\lim _{j \rightarrow \infty} \mathcal{E}_{s_{j}}^{\infty}=\lim _{j \rightarrow \infty} \mathcal{E}_{s_{j} t_{i_{j}}}=$ $\mathcal{E}^{\infty, \infty}$. (The rescaling in $\mathcal{E}_{s_{j}}^{\infty}$ involves pullback with respect to $u \rightarrow s_{j} u$ and a $j$-dependent diffeomorphism $\phi_{j}$ of $X^{\infty}$, along with a $j$-dependent automorphism of the flat $\mathbb{R}$-vector bundle on $X^{\infty}$.) From Assumption 3.34, the orbit space of $X^{\infty, \infty}$ is two dimensional. The Lorentzian metric corresponding to the groupoid Einstein flow $\mathcal{E}^{\infty, \infty}$ can be locally written as

$$
-\left(L^{\infty}\right)^{2}\left(d u^{\infty}\right)^{2}+\sum_{\alpha, \beta=1}^{2} h_{\alpha \beta}^{\infty} d b_{\alpha}^{\infty} d b_{\beta}^{\infty}+G^{\infty}\left(d \theta+A^{\infty}\right)^{2} .
$$

We will show this is an Einstein flow of Bianchi-III flat type and take $t_{j}^{\prime}=s_{j} t_{i_{j}}$.

Put

$$
g^{\infty}=-\left(L^{\infty}\right)^{2}\left(d u^{\infty}\right)^{2}+\sum_{\alpha, \beta=1}^{2} h_{\alpha \beta}^{\infty} d b_{\alpha}^{\infty} d b_{\beta}^{\infty},
$$

$\widehat{g}^{\infty}=G^{\infty} g^{\infty}$ and $\widehat{G}^{\infty}=\left(G^{\infty}\right)^{2}$. Then

$$
g^{\infty}+G^{\infty}(d \theta+A)^{2}=\left(\widehat{G}^{\infty}\right)^{-\frac{1}{2}}\left(\widehat{g}^{\infty}+\widehat{G}^{\infty}\left(d \theta+A^{\infty}\right)^{2}\right) .
$$

Let $\widehat{u}_{s_{j}}$ be $\frac{1}{s_{j}}$ times the pullback of $\widehat{u}$ with respect to $u \rightarrow s_{j} u$ and $\phi_{j} \in \operatorname{Diff}\left(X^{\infty}\right)$. From Assumption 3.46 $(1,2)$, after passing to a subsequence we can assume that $\lim _{j \rightarrow \infty} \widehat{u}_{s_{j}}=\widehat{u}^{\infty}$ in the weak topology on $W_{l o c}^{3, p}$, for some $\widehat{u}^{\infty} \in W_{l o c}^{3, p}\left((0, \infty) \times X^{\infty, \infty}\right)$. From Assumption 3.38 and Assumption 3.46(3), the gradient $\nabla \widehat{u}^{\infty}$ is timelike, and the level sets of $\widehat{u}^{\infty}$ have constant mean curvature with respect to $\widehat{g}^{\infty}$.

We will apply the monotonicity result of Subsection A.4 with $n=2$ and $N=1$, to $\mathcal{E}^{\infty}$, replacing the $g$ and $G$ of Subsection A.4 by $\widehat{g}$ and $\widehat{G}$. Let $\widehat{h}$ denote the induced metric on the level sets $\widehat{X}^{\infty}$ of $\widehat{u}$. Let $\widehat{H}$ denote the (constant) mean curvatures of the level sets. From Subsection A.4, we know that $(-\widehat{H})^{2} \operatorname{vol}\left(\widehat{X}^{\infty}, \widehat{h}(\widehat{u})\right)$ is nonincreasing in $\widehat{u}$. It is 
clearly nonnegative. Since $\operatorname{dim}\left(\widehat{X}^{\infty}\right)=2$, the expression is invariant under rescaling. Note that when forming the $\operatorname{limit}_{\lim _{j \rightarrow \infty}} \mathcal{E}_{s_{j}}^{\infty}=\mathcal{E}^{\infty, \infty}$, we are allowed to perform $j$-dependent automorphisms of the flat 1-dimensional vector bundle on $X^{\infty}$. These automorphisms can change $G$ by a $j$-dependent multiplicative constant, and hence change $\widehat{g}$ by a multiplicative constant. One sees that on a given level set, this does not change $(-\widehat{H})^{2} \operatorname{vol}\left(\widehat{X}^{\infty}, \widehat{h}\right)$.

Given $a \in(0, \infty)$, the level set $\left(\widehat{u}^{\infty}\right)^{-1}(a) \subset(0, \infty) \times X^{\infty, \infty}$ is the limit of rescalings of level sets $\widehat{u}^{-1}\left(s_{j} a\right) \subset(0, \infty) \times X^{\infty}$. It follows that the monotonic quantity $\left(-\widehat{H}^{\infty}\right)^{2} \operatorname{vol}\left(\widehat{X}^{\infty, \infty}, \widehat{h}^{\infty}\right)$ is constant in $\widehat{u}^{\infty}$. By Subsection A.4, we conclude that $\mathcal{E}^{\infty, \infty}$ is an Einstein flow of Bianchi-III flat type.

Let $\widetilde{X}$ denote the universal cover of $X$. We give it the pullback Einstein flow.

Corollary 3.53. Under the hypotheses of Proposition 3.49 , choose $\widetilde{x}_{j}^{\prime} \in \widetilde{X}$. Then $\left\{\left(\widetilde{\mathcal{E}}_{t_{j}^{\prime}}, \widetilde{x}_{j}^{\prime}\right)\right\}_{j=1}^{\infty}$ approaches the set of Bianchi-III flat Einstein flows on $\mathbb{R}^{3}$, in the pointed weak $W^{2, p}$ topology and the pointed $C^{1, \alpha}$-topology.

Proof. Given Proposition 3.49, the corollary follows as in [22, Section 6.2].

Corollary 3.54. Under the hypotheses of Proposition 3.49, X has Thurston type $H^{2} \times \mathbb{R}$ or $\mathrm{SL}(2, \mathbb{R})$.

Proof. The sequence $\left\{\left(t_{j}^{\prime}\right)^{-2} h\left(t_{j}^{\prime}\right)\right\}_{j=1}^{\infty}$ of Riemannian metrics on $X$ Gromov-Hausdorff converges with bounded curvature to a two dimensional compact hyperbolic orbifold, from which the corollary follows.

\section{TyPE-II BLOWDOWN}

Let $\mathcal{E}$ be an expanding CMC Einstein flow that is not type-III in the sense of Definition 2.53. Then we say that $\mathcal{E}$ is a type-IIb Einstein flow. One can get information about such a flow by a rescaling analysis. The rescaling now involves the size of the curvature tensor, unlike in the type-III case where the rescaling involves the Hubble time.

After rescaling and passing to a limit, one obtains an Einstein flow $\mathcal{E}^{\infty}$ on an étale groupoid, defined for times $t \in \mathbb{R}$, with vanishing mean curvature. We show that if the second fundamental form of the original flow $\mathcal{E}$ is controlled by the mean curvature, then $\mathcal{E}^{\infty}$ is the static flow on a Ricci-flat Riemannian groupoid. In particular, if $\mathcal{E}$ is locally homogeneous or on a three-dimensional manifold, then $\mathcal{E}^{\infty}$ is flat. This may seem to contradict the fact that the rescalings in the blowdown procedure normalize the size of the curvature tensor, but the point is that the convergence to $\mathcal{E}^{\infty}$ is in the weak $W^{2, p}$-topology.

Relevant example come from the homogeneous Einstein flows on $\widetilde{\mathrm{SL}(2, \mathbb{R})}$ considered in [25].

More generally, we show that if $\mathcal{E}$ is a type-IIb Einstein flow on a three dimensional manifold then the second fundamental form fails to be controlled by the mean curvature, or the first covariant derivative of the curvature tensor fails to be controlled by the curvature norm. 
To begin, let $\mathcal{E}$ be a type-IIb Einstein flow on a compact $n$-dimensional manifold. Given $t \in\left[t_{0}, \infty\right)$, let $x_{t} \in X$ be a point where the time- $t$ curvature norm $|\mathrm{Rm}|_{T}$ is maximized.

Proposition 4.1. We can find a sequence $\left\{t_{i}\right\}_{i=1}^{\infty}$ with $\lim _{i \rightarrow \infty} t_{i}=\infty$ such that the following property holds. Put $Q_{i}=|\operatorname{Rm}|_{T}\left(x_{i}, t_{i}\right)$ and $\mathcal{E}^{(i)}(u)=\mathcal{E}_{Q_{i}^{-\frac{1}{2}}}\left(u+Q_{i}^{\frac{1}{2}} t_{i}\right)$. Then after passing to a subsequence, there is a limit $\lim _{i \rightarrow \infty}\left(\mathcal{E}^{(i)}, x_{i}\right)=\left(\mathcal{E}^{\infty}, x_{\infty}\right)$ in the pointed weak $W^{2, p}$-topology and the pointed $C^{1, \alpha}$-topology. Here $\mathcal{E}^{\infty}$ is an Einstein flow on an $n$-dimensional étale groupoid, defined for $t \in \mathbb{R}$. If there is some $C<\infty$ such that $|K|^{2} \leq C H^{2}$ then $L^{\infty}$ is uniformly bounded below by a positive constant.

Proof. As in [12, Chapter 8.2.1.3], we can make an initial choice of the $t_{i}$ 's so that $\lim _{i \rightarrow \infty} Q_{i} t_{i}^{2}=$ $\infty$ and for any compact time interval $S \subset \mathbb{R}$, there are bounds on $|\operatorname{Rm}|_{T}$ on $S$ for the rescaled flows $\left\{\mathcal{E}^{(i)}\right\}_{i=1}^{\infty}$ that are uniform in $i$. This implies uniform bounds on $S$ for $\left|K^{(i)}\right|$ [2, Proposition 2.2]. The rescaled Einstein flow $\mathcal{E}^{(i)}$ has

$$
\begin{aligned}
\left|\mathrm{Rm}^{(i)}\right|_{T}(u) & =Q_{i}^{-1}|\operatorname{Rm}|_{T}\left(Q_{i}^{-\frac{1}{2}} u+t_{i}\right), \\
\left|K^{(i)}\right|(u) & =Q_{i}^{-\frac{1}{2}}|K|\left(Q_{i}^{-\frac{1}{2}} u+t_{i}\right), \\
H^{(i)}(u) & =-\frac{n Q_{i}^{-\frac{1}{2}}}{Q_{i}^{-\frac{1}{2}} u+t_{i}} .
\end{aligned}
$$

From Proposition [3.2, after passing to a subsequence there is a $\operatorname{limit} \lim _{i \rightarrow \infty}\left(\mathcal{E}^{(i)}, x_{i}\right)=$ $\left(\mathcal{E}^{\infty}, x_{\infty}\right)$ as stated. If $|K(t)|^{2} \leq C H(t)^{2}=C \frac{n^{2}}{t^{2}}$ then

$$
\left|K^{(i)}\right|^{2}(u)=Q_{i}^{-1}|K|^{2}\left(Q_{i}^{-\frac{1}{2}} u+t_{i}\right) \leq C Q_{i}^{-1} \frac{n^{2}}{\left(Q_{i}^{-\frac{1}{2}} u+t_{i}\right)^{2}}=\frac{C}{n} \frac{\partial H^{(i)}(u)}{\partial u} .
$$

From Proposition 3.2, the lapse function $L^{\infty}$ is positive. As in the proof of Corollary 2.54, it is uniformly bounded below by a positive constant. (It is bounded above by one.)

As noted in [2, Section 5], because of the renormalization, the flow $\mathcal{E}^{\infty}$ has vanishing mean curvature $H^{\infty}$, since $\lim _{i \rightarrow \infty} H^{(i)}(u)=-\lim _{i \rightarrow \infty} \frac{n}{u+Q_{i}^{\frac{1}{2}} t_{i}}=0$.

Proposition 4.4. If a type-IIb expanding CMC Einstein flow $\mathcal{E}$ has a uniform upper bound on $\frac{|K|^{2}}{H^{2}}$ then the blowdown limit $\mathcal{E}^{\infty}$ is a static Einstein flow on a Ricci-flat Riemannian groupoid.

Proof. Because of the rescaling, the blowdown limit has vanishing second fundamental form $K^{\infty}$. Hence $\mathcal{E}^{\infty}$ is a static Einstein flow on a Riemannian groupoid $\left(\mathcal{X}^{\infty}, h^{\infty}\right)$. The static Einstein flow equations become $L^{\infty} R_{i j}^{\infty}=L_{; i j}^{\infty}$ and $\triangle_{h^{\infty}} L^{\infty}=0$. In the smooth structure on the unit space of $\mathcal{E}^{\infty}$ coming from local harmonic coordinates, by elliptic regularity the metric $h^{\infty}$ is smooth and $L^{\infty}$ is smooth. We use the trick from [1, Appendix] of passing to $\mathcal{Y}^{\infty}=\mathcal{X}^{\infty} \times S^{1}$ with the Riemannian metric $h^{\infty}+\left(L^{\infty}\right)^{2} d \theta^{2}$, which is Ricci-flat. The function $\log L^{\infty}$ is a bounded harmonic function on $\mathcal{Y}^{\infty}$. The proof of [29, Corollary 1] 
extends to the groupoid setting to show that $L^{\infty}$ is constant. Then $h^{\infty}$ is Ricci-flat. This shows that $\mathcal{E}^{\infty}$ is a static Einstein flow on a Ricci-flat Riemannian groupoid $\mathcal{X}^{\infty}$, thereby proving the proposition.

Corollary 4.5. Under the hypotheses of Proposition 4.4. if $X$ is locally homogeneous then the type-IIb blowdown limit $\mathcal{E}^{\infty}$ is a static Einstein flow on a flat Riemannian groupoid.

Proof. A Gromov-Hausdorff limit of homogeneous spaces is still homogeneous [17, p. 66]. Applying this to the balls in the tangent spaces, it follows that $\left(X^{\infty}, h^{\infty}(t)\right)$ is a locally homogeneous Ricci-flat Riemannian groupoid, and hence is flat [28].

Corollary 4.6. Under the hypotheses of Proposition 4.4, if $n=3$ then the type-IIb blowdown limit $\mathcal{E}^{\infty}$ is a static Einstein flow on a flat Riemannian groupoid.

Proof. A Ricci-flat three dimensional Riemannian groupoid is flat.

It may seem contradictory that although we rescale so that the norm of the curvature tensor at $\left(x_{i}, t_{i}\right)$ is one, the limit flow is flat. The point is that the convergence to the limit flow is in the weak $W^{2, p}$-topology, which does not imply pointwise convergence of the curvature norm. In effect, there are increasing fluctuations of the curvature tensor, which average it out to zero.

Under the hypotheses of Corollary 4.6, one does have pointed $C^{1, \alpha}$-convergence of the normalized Einstein flows to the flat limit flow. In particular, put $\widehat{h}_{i}=Q_{i} \exp _{x_{i}}^{*} h\left(t_{i}\right)$, a metric defined at least on $B_{i}=B\left(0, \pi Q_{i}^{-\frac{1}{2}}\right) \subset T_{x_{i}} X$. Then the pointed balls $\left(B_{i}, x_{i}, \widehat{h}_{i}\right)$ converge in the sense of distance geometry, i.e. in the pointed Gromov-Hausdorff topology, to the flat Euclidean metric on a three dimensional ball of radius $\pi$. However, their curvature tensors do not converge.

Corollary 4.7. When $n=3$, if there is some $C<\infty$ so that an expanding $C M C$ Einstein flow has $|K|^{2} \leq C H^{2}$ and $|\nabla \mathrm{Rm}|_{T}(x, t) \leq C \sup _{y \in X}|R m|_{T}^{\frac{3}{2}}(y, t)$ for all $x \in X$ and $t \in$ $\left[t_{0}, \infty\right)$, then the flow must be type-III.

Proof. If $\mathcal{E}$ is not type-III then Corollary 4.6 applies. From the bound on the normalized covariant derivative of the curvature tensor, we have convergence of the normalized Einstein flows to $\mathcal{E}^{\infty}$ in the pointed weak $W^{3, p}$-topology. This implies pointwise convergence of the curvature tensors. The normalized curvature tensors of $\mathcal{E}$ have norm 1 at $\left(x_{i}, t_{i}\right)$, but converge to the vanishing curvature of $\mathcal{E}^{\infty}$ at $\left(x_{\infty}, 0\right)$, which is a contradiction.

Remark 4.8. Corollary 4.7 can be proven by just working on balls in tangent spaces, instead of dealing with étale groupoids.

Example 4.9. An example of a type-IIb Einstein flow was given in [25]. Consider SL $\widetilde{\mathrm{L}(2, \mathbb{R})}$

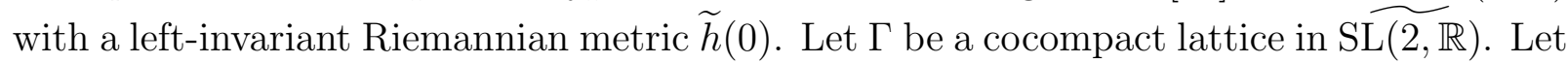
$h(0)$ be the quotient metric on $X=\Gamma \backslash \widehat{\mathrm{SL}(2, \mathbb{R})}$. Let $\widetilde{K}(0)$ be a left-invariant symmetric covariant 2-tensor field on $\widetilde{\mathrm{SL}(2, \mathbb{R})}$. Let $K(0)$ be the quotient 2-tensor field on $X$. Let $\mathcal{E}$ be the ensuing Einstein flow on $X$, with initial conditions $(h(0), K(0))$. 
Let $\mathbb{R} \subset \mathrm{SL}(2, \mathbb{R})$ be the lift of $\mathrm{SO}(2) \subset \mathrm{SL}(2, \mathbb{R})$. If $(h(0), K(0))$ is right- $\mathbb{R}$ invariant then $\mathcal{E}$ is type-III. Otherwise, it is type-IIb [25].

In the latter case, we claim that Corollary 4.6 applies. This follows from results in [25, Pf. of Theorem 3]. In the notation there, the normalized traceless part $\frac{K^{0}}{H}$ of the second fundamental form is determined by $\Sigma_{ \pm}$. It is shown that $\Sigma_{ \pm}$are uniformly bounded in $t$. Hence the blowdown Einstein flow is the static flow on a flat Riemannian groupoid.

We claim that this limit groupoid is $\mathbb{R}^{2} \times\left(\mathbb{R} \rtimes \mathbb{R}_{\delta}\right)$, where $\mathbb{R}_{\delta}$ denotes $\mathbb{R}$ with the discrete topology. From [24, Theorem 3], we can write $h(t)=\sum_{i=1}^{3} a_{i}^{2}(t) \xi^{i} \otimes \xi^{i}$ with $a_{1}(t) \sim \alpha_{1}(\ln t)^{\frac{1}{2}}$ and $a_{i}(t) \sim \alpha_{i} t$ for $i \in\{2,3\}$. Here $\alpha_{1}, \alpha_{2}, \alpha_{3}>0$. From [25, Theorem 3], we have $|\operatorname{Rm}|_{T}(t) \sim \frac{c_{0}}{t \ln t}$ for some $c_{0}>0$. Hence the normalized lengths are comparable

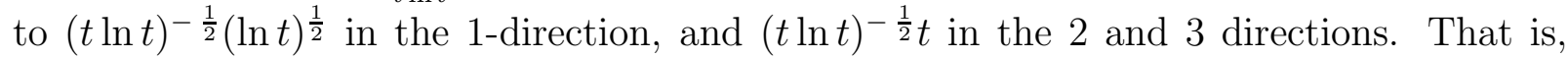
there are two expanding directions and one shrinking direction. Then the limit flat étale groupoid must be $\mathbb{R}^{2} \times\left(\mathbb{R} \rtimes \mathbb{R}_{\delta}\right)$.

The fact that there are increasing fluctuations of the curvature tensor, which cause its averaging out to zero, is consistent with the nonuniform behavior shown in [25, Theorem 3 and Proposition 2].

\section{Appendix A. Monotonicity FORMulas}

In this section we derive monotonicity formulas for dimensionally reduced Einstein flows. We consider a coupled system on a connected compact manifold $B$, where the fields on $B$ are (locally) a Lorentzian metric $g$, an $\mathbb{R}^{N}$-valued connection $A$ and a map $G$ to positive definite $(N \times N)$-matrices. Such coupled systems arise, for example, when doing dimensional reduction of the vacuum Einstein equation on a manifold $M$ with a free $T^{N}$-action, and quotient space $B$. The vacuum Einstein equation on $M$ becomes a coupled system consisting of a nonvacuum Einstein equation for $g$, a Yang-Mills-type equation for $A$ and a wave-type equation for $G$. In Subsection A.1 we write the equations and begin their analysis.

Subsections A.2 and A.3 are concerned with monotonicity formulas when there is a spacetime decomposition for which $\operatorname{det}(G)$ is constant along spatial hypersurfaces. In [5] and related papers, such a spacetime decomposition is said to provide "areal" coordinates. It is especially relevant when $\operatorname{dim}(B)=2$.

Subsection A.4 deals with a monotonic quantity that exists when $\operatorname{dim}(B)>2$. In the case when $\operatorname{dim}(B)=3$ and $N=1$, it reduces to the "first energy" of Choquet-Bruhat [10] and Choquet-Bruhat-Moncrief [11].

More detailed descriptions are given at the beginnings of the subsections.

The results of this appendix extend in a straightforward way to the setting where $B$ is an orbifold. In the appendix, we only consider the case when $B$ is a manifold.

A.1. Curvature formulas under an $\mathbb{R}^{N}$-symmetry. We begin with the geometric setup of [22, Section 4.1], to which we refer for more details. Let $\mathcal{G}$ be an $N$-dimensional abelian Lie group, with Lie algebra $\mathfrak{g}$. Let $\mathfrak{E}$ be a local system on $B$ of Lie groups isomorphic to $\mathcal{G}$. There is a corresponding flat $\mathfrak{g}$-vector bundle $e$ on $B$; see [22, Section 4.1]. 
Let $M$ be the total space of an $\mathfrak{E}$-twisted principal $\mathcal{G}$-bundle with base $B$, in the sense of [22, Section 4.1]. (An example is when $\mathfrak{E}$ is the constant local system and $M$ is the total space of a $T^{N}$-bundle on $B$.) We write $\operatorname{dim}(B)=n+1$ and $\operatorname{dim}(M)=m=N+n+1$.

Let $\bar{g}$ be a Lorentzian metric on $M$ with a free local isometric $\mathfrak{E}$-action. We assume that the induced metrics on the $\mathfrak{E}$-orbits are Riemannian. In adapted coordinates, we can write

$$
\bar{g}=\sum_{I, J=1}^{N} G_{I J}\left(d x^{I}+A^{I}\right)\left(d x^{J}+A^{J}\right)+\sum_{\alpha, \beta=1}^{n+1} g_{\alpha \beta} d b^{\alpha} d b^{\beta} .
$$

Here $G_{I J}$ is the local expression of a Euclidean inner product on $e, \sum_{\alpha, \beta=1}^{n+1} g_{\alpha \beta} d b^{\alpha} d b^{\beta}$ is the local expression of a Lorentzian metric $g_{B}$ on $B$ and $A^{I}=\sum_{\alpha} A_{\alpha}^{I} d b^{\alpha}$ are the components of a local $e$-valued 1-form describing an connection $A$ on the twisted $\mathfrak{G}$-bundle $M \rightarrow B$.

Put $F_{\alpha \beta}^{I}=\partial_{\alpha} A_{\beta}^{I}-\partial_{\beta} A_{\alpha}^{I}$. At a given point $b \in B$, we can assume that $A^{I}(b)=0$. We write

$$
G_{I J ; \alpha \beta}=G_{I J ; \alpha \beta}-\Gamma_{\alpha \beta}^{\sigma} G_{I J, \sigma},
$$

where $\left\{\Gamma_{\alpha \beta}^{\sigma}\right\}$ are the Christoffel symbols for the metric $g_{\alpha \beta}$ on $B$.

From [22, Section 4.2], the Ricci tensor of $\bar{g}$ on $M$ is given in terms of the curvature tensor $R_{\alpha \beta \gamma \delta}$ of $B$, the 2 -forms $F_{\alpha \beta}^{I}$ and the metrics $G_{I J}$ by

$$
\begin{aligned}
\bar{R}_{I J}^{\bar{g}}= & -\frac{1}{2} g^{\alpha \beta} G_{I J ; \alpha \beta}-\frac{1}{4} g^{\alpha \beta} G^{K L} G_{K L, \alpha} G_{I J, \beta}+\frac{1}{2} g^{\alpha \beta} G^{K L} G_{I K, \alpha} G_{L J, \beta}+ \\
& \frac{1}{4} g^{\alpha \gamma} g^{\beta \delta} G_{I K} G_{J L} F_{\alpha \beta}^{K} F_{\gamma \delta}^{L} \\
\bar{R}_{I \alpha}^{\bar{g}}= & \frac{1}{2} g^{\gamma \delta} G_{I K} F_{\alpha \gamma ; \delta}^{K}+\frac{1}{2} g^{\gamma \delta} G_{I K, \gamma} F_{\alpha \delta}^{K}+\frac{1}{4} g^{\gamma \delta} G_{I m} G^{K L} G_{K L, \gamma} F_{\alpha \delta}^{m} \\
\bar{R}_{\alpha \beta}^{\bar{g}}= & R_{\alpha \beta}^{g}-\frac{1}{2} G^{I J} G_{I J ; \alpha \beta}+\frac{1}{4} G^{I J} G_{J K, \alpha} G^{K L} G_{L I, \beta}-\frac{1}{2} g^{\gamma \delta} G_{I J} F_{\alpha \gamma}^{I} F_{\beta \delta}^{J} .
\end{aligned}
$$

The scalar curvature is

$$
\begin{aligned}
\bar{R}^{\bar{g}}= & R^{g}-g^{\alpha \beta} G^{I J} G_{I J ; \alpha \beta}+\frac{3}{4} g^{\alpha \beta} G^{I J} G_{J K, \alpha} G^{K L} G_{L I, \beta} \\
& -\frac{1}{4} g^{\alpha \beta} G^{I J} G_{I J, \alpha} G^{K L} G_{K L, \beta}-\frac{1}{4} g^{\alpha \gamma} g^{\beta \delta} G_{I J} F_{\alpha \beta}^{I} F_{\gamma \delta}^{J} .
\end{aligned}
$$

In what follows we will assume that the flat vector bundle $e$ has holonomy in $\operatorname{SL}(N, \mathbb{R})$, so that $\ln \operatorname{det} G$ is globally defined on $B$. We have

$$
\nabla_{\alpha} \ln \operatorname{det} G=G^{I J} G_{I J, \alpha}
$$

and

$$
\triangle_{g} \ln \operatorname{det} G=g^{\alpha \beta} G^{I J} G_{I J ; \alpha \beta}-g^{\alpha \beta} G^{I J} G_{J K, \alpha} G^{K L} G_{L K, \beta} .
$$

Writing

$$
|F|^{2}=G_{I J} g^{\alpha \beta} g^{\gamma \delta} F_{\alpha \gamma}^{I} F_{\beta \delta}^{J},
$$


the first equation in (A.3) gives

$$
G^{I J} \bar{R}_{I J}=-\frac{1}{2} \triangle_{g} \ln \operatorname{det} G-\frac{1}{4} g^{\alpha \beta}\left(\nabla_{\alpha} \ln \operatorname{det} G\right)\left(\nabla_{\beta} \ln \operatorname{det} G\right)+\frac{1}{4}|F|^{2} .
$$

Note that $|F|^{2}$ need not be nonnegative.

Given a foliation of $B$ by compact spacelike hypersurfaces $Y$, we can write the metric $g$ on $B$ as

$$
g=-L^{2} d t^{2}+\sum_{i, j=1}^{n} h_{i j} d y^{i} d y^{j}
$$

Here $L=L(y, t)$ is the lapse function and we have performed spatial diffeomorphisms to kill the shift vectors.

A.2. Monotonicity formulas for equivolume foliations. In this subsection we introduce a first monotonicity formula for equivolume foliations. Suppose that $\operatorname{det} G$ is spatially constant, i.e. only depends on $t$. Then

$$
g^{\alpha \beta}\left(\nabla_{\alpha} \ln \operatorname{det} G\right)\left(\nabla_{\beta} \ln \operatorname{det} G\right)=-L^{-2}\left(\partial_{t} \ln \operatorname{det} G\right)^{2}
$$

and

$$
\triangle_{g} \ln \operatorname{det} G=-\frac{1}{L \sqrt{\operatorname{det} h}} \partial_{t}\left(L^{-1} \sqrt{\operatorname{det} h}\left(\partial_{t} \ln \operatorname{det} G\right)\right) .
$$

If $\bar{R}_{I J}^{\bar{g}}=0$ then (A.8) becomes

$$
0=\frac{1}{2} \frac{1}{L \sqrt{\operatorname{det} h}} \partial_{t}\left(L^{-1} \sqrt{\operatorname{det} h}\left(\partial_{t} \ln \operatorname{det} G\right)\right)+\frac{1}{4} L^{-2}\left(\partial_{t} \ln \operatorname{det} G\right)^{2}+\frac{1}{4}|F|^{2} .
$$

Multiplying by $L \sqrt{\operatorname{det} h}$ and integrating over $Y$ gives

$$
\begin{aligned}
\frac{\partial}{\partial t}\left(\left(\partial_{t} \ln \operatorname{det} G\right) \int_{Y} L^{-1} \mathrm{dvol}_{Y}\right)= & -\frac{1}{2}\left(\partial_{t} \ln \operatorname{det} G\right)^{2} \int_{Y} L^{-1} \mathrm{dvol}_{Y} \\
& -\frac{1}{2} \int_{Y}|F|^{2} L \operatorname{dvol}_{Y}
\end{aligned}
$$

If $F=0$ then $\left(\partial_{t} \ln \operatorname{det} G\right) \int_{Y} L^{-1} \operatorname{dvol}_{Y}$ is monotonically nonincreasing in $t$.

A.3. Two dimensions. In this subsection we specialize to the case when $\operatorname{dim}(B)=2$. We begin with some generalities. In Subsubsection A.3.1 we consider monotonic quantities in the case $F=0$. Besides the monotonic quantity of Subsection A.2, we analyze an energy-like monotonic functional $\widehat{\mathcal{E}}$.

In Subsubsection A.3.2 we look at the case when $F$ is nonzero. In order to apply results from the literature, in that subsubsection we specialize to the case $N=2$. We introduce the monotonic quantity $\widehat{\mathcal{E}}_{K}$ and show that it is well-defined no matter what the global twisting $H \in \mathrm{SL}(2, \mathbb{R})$ may be. We characterize when $\widehat{\mathcal{E}}_{K}$ is constant in $t$. 
Continuing with Subsection A.2, suppose that $\operatorname{dim}(B)=2$, i.e. $\operatorname{dim}(Y)=1$. We write $g$ locally (in $Y$ ) as $-L^{2} d t^{2}+h d y^{2}$. We have $R_{\alpha \beta}^{g}=\frac{1}{2} R g_{\alpha \beta}$, so $g^{t t} R_{t t}^{g}=g^{y y} R_{y y}^{g}$. Hence $-L^{-2} R_{t t}^{g}=h^{-1} R_{y y}^{g}$. If $\bar{R}_{\alpha \beta}^{\bar{g}}=0$ then the third equation of (A.3) gives

$$
\begin{aligned}
& L^{-2} \operatorname{Tr}\left(G^{-\frac{1}{2}} G_{, t} G^{-\frac{1}{2}}\right)^{2}+h^{-1} \operatorname{Tr}\left(G^{-\frac{1}{2}} G_{, y} G^{-\frac{1}{2}}\right)^{2}= \\
& L^{-2} \operatorname{Tr}\left(G^{-1} G_{, t}\right)^{2}+h^{-1} \operatorname{Tr}\left(G^{-1} G_{, y}\right)^{2}= \\
& -2 L^{-2}(\ln \operatorname{det} G)_{; t}=-2 L^{-2}(\ln \operatorname{det} G)_{t t}+2 L^{-3} L_{t}(\ln \operatorname{det} G)_{t} .
\end{aligned}
$$

If in addition $(\ln \operatorname{det} G)_{t}=0$ then from (A.14),$G^{-\frac{1}{2}} G_{, t} G^{-\frac{1}{2}}$ and $G^{-\frac{1}{2}} G_{, y} G^{-\frac{1}{2}}$ vanish, so $G$ is locally constant in $y$ and $t$. Then the third equation of (A.3) gives $R_{\alpha \beta}^{g}=0$, so $B$ is flat. The holonomy around $Y$ of the flat vector bundle $e$ must be orthogonal.

A.3.1. Gowdy spacetime. In this subsubsection we assume that $F=0$. From (A.13), $\left(\partial_{t} \ln \operatorname{det} G\right) \int_{Y} L^{-1} \mathrm{dvol}_{Y}$ is monotonically nonincreasing in $t$. If it is constant in $t$ then the right-hand side of (A.13) vanishes, so $\partial_{t} \ln \operatorname{det} G=0$. Hence $G$ is locally constant in $y$ and $t$, and $B$ is flat.

For another monotonic quantity, consider

$$
\begin{aligned}
\mathcal{E}(t) & =\int_{Y}\left[h^{-1} \operatorname{Tr}\left(\left(G^{-1} \frac{\partial G}{\partial y}\right)^{2}\right)+L^{-2} \operatorname{Tr}\left(\left(G^{-1} \frac{\partial G}{\partial t}\right)^{2}\right)\right] L \text { dvol } \\
& =\int_{Y}\left[L h^{-\frac{1}{2}} \operatorname{Tr}\left(\left(G^{-1} \frac{\partial G}{\partial y}\right)^{2}\right)+L^{-1} h^{\frac{1}{2}} \operatorname{Tr}\left(\left(G^{-1} \frac{\partial G}{\partial t}\right)^{2}\right)\right] d y .
\end{aligned}
$$

Still assuming that $F=0$, equation (A.12) gives

$$
(\ln \operatorname{det} G)_{t} \partial_{t}\left(L h^{-\frac{1}{2}}\right)=L h^{-\frac{1}{2}}\left((\ln \operatorname{det} G)_{t t}+\frac{1}{2}(\ln \operatorname{det} G)_{t}^{2}\right) .
$$

When $\bar{R}_{I J}^{\bar{g}}=0$, equation (A.3) gives the matrix equation

$$
\begin{aligned}
& -L^{-2}\left(G^{-1} G_{t t}-G^{-1} G_{t} G^{-1} G_{t}\right)+h^{-1}\left(G^{-1} G_{y y}-G^{-1} G_{y} G^{-1} G_{y}\right)+ \\
& L^{-3} L_{t} G^{-1} G_{t}+L^{-1} h^{-1} L_{y} G^{-1} G_{y}-\frac{1}{2} L^{-2} h^{-1} h_{t} G^{-1} G_{t}- \\
& \frac{1}{2} h^{-2} h_{y} G^{-1} G_{y}-\frac{1}{2} L^{-2}(\ln \operatorname{det} G)_{t} G^{-1} G_{t}=0 .
\end{aligned}
$$

Using (A.16) and (A.17), one finds

$$
\begin{aligned}
\frac{d}{d t}\left((\ln \operatorname{det} G)_{t} \mathcal{E}\right)= & \left(2(\ln \operatorname{det} G)_{t t}+\frac{1}{2}(\ln \operatorname{det} G)_{t}^{2}\right) \mathcal{E}- \\
& \frac{1}{2}(\ln \operatorname{det} G)_{t}^{2} \int_{Y} L^{-1} \operatorname{Tr}\left(\left(G^{-1} G_{t}\right)^{2}\right) \text { dvol }
\end{aligned}
$$


If $(\ln \operatorname{det} G)_{t} \neq 0$ then a scale invariant quantity is given by

$$
\widehat{\mathcal{E}}(t)=\frac{2}{(\ln \operatorname{det} G)_{t} \sqrt{\operatorname{det} G}} \mathcal{E}(t)
$$

Using (A.18), one finds

$$
\frac{d \widehat{\mathcal{E}}}{d t}=-\frac{1}{\sqrt{\operatorname{det} G}} \int_{Y} L^{-1} \operatorname{Tr}\left(\left(G^{-1} G_{t}\right)^{2}\right) \text { dvol }
$$

If the right-hand side of (A.20) vanishes then $G$ is constant in $t$. As before, this implies that $G$ is constant in $y$ and $t$, and $B$ is flat.

Remark A.21. If we use the areal time variable $t=\sqrt{\operatorname{det} G}$ then $\widehat{\mathcal{E}}(t)=\mathcal{E}(t)$ and

$$
\frac{d \widehat{\mathcal{E}}}{d t}=-\frac{1}{t} \int_{Y} L^{-1} \operatorname{Tr}\left(\left(G^{-1} G_{t}\right)^{2}\right) \mathrm{dvol}
$$

compare with (A.26) and (A.27).

A.3.2. NonGowdy spacetime. We now assume that $F \neq 0$. If $\bar{R}_{I \alpha}^{\bar{g}}=0$ then from the second equation in (A.3), one finds that the $\mathbb{R}^{N}$-valued vector

$$
C_{I}=L^{-1} h^{-\frac{1}{2}} \sqrt{\operatorname{det} G} G_{I K} F_{t y}^{K}
$$

is locally constant on the two dimensional spacetime. More precisely, it is a locally constant section of the flat vector bundle $e^{*}$ (using our assumption that $e$ is unimodular).

We now restrict to the case when $N=2$ and the flat $\mathbb{R}^{2}$-bundle $e$ has holonomy $H$, around the circle $Y$, lying in $\operatorname{SL}(2, \mathbb{R})$. When $H=\mathrm{Id}$, the components of $C$ are called the "twist quantities" in [5] and subsequent papers such as [20]. We mostly follow the notation of [20, p. 1256-1283], with coordinates $(R, \theta)$ for the two dimensional base. We use linear coordinates $x^{1}, x^{2}$ for the $\mathbb{R}^{2}$-fiber. In that paper, $R=\operatorname{det} G$ and $\theta$ is the coordinate for the spacelike hypersurface $Y$. The coordinates $x^{1}$ and $x^{2}$ are chosen so that $C_{1}=0$ and $C_{2}=K$, where $K$ is a constant. The Lorentzian metric on $(0, \infty) \times Y$ can be written as

(A.24) $g=e^{2(\eta-U)}\left(-d R^{2}+a^{-2} d \theta^{2}\right)+e^{2 U}\left(d x^{1}+A d x^{2}+(G+A H) d \theta\right)^{2}+e^{-2 U} R^{2}\left(d x^{2}+H d \theta\right)^{2}$.

Put

$$
\mathcal{D}=a^{-1} U_{R}^{2}+a U_{\theta}^{2}+R^{-2} e^{4 U}\left(a^{-1} A_{R}^{2}+a A_{\theta}^{2}\right)
$$

and

$$
\widehat{\mathcal{E}}_{K}(R)=\int_{Y}\left(\mathcal{D}+\frac{1}{4} K^{2} R^{-4} e^{2 \eta} a^{-1}\right) d \theta .
$$

Then from [20, p. 1283]

$$
\frac{d \widehat{\mathcal{E}}_{K}}{d R}=-2 R^{-1} \int_{Y}\left(a^{-1} U_{R}^{2}+\frac{1}{4} R^{-2} e^{4 U} a A_{\theta}^{2}\right) d \theta-\frac{1}{2} K^{2} R^{-3} \int_{Y} \mathcal{D} e^{2 \eta} d \theta .
$$


If $t$ is a time variable, with $R$ a monotonically increasing function of $t$, then

$$
\frac{d \widehat{\mathcal{E}}_{K}}{d t}=\frac{(\operatorname{det} G)_{t}}{2 \sqrt{\operatorname{det} G}} \frac{d \widehat{\mathcal{E}}_{K}}{d R} .
$$

The quantity $\widehat{\mathcal{E}}_{K}$ is scale invariant.

To treat the more general case when $H \in \mathrm{SL}(2, \mathbb{R})$, since $C$ is a nonzero flat section of $e^{*}$, the matrix $H^{-T}$ must be unipotent, i.e. conjugate to $\left(\begin{array}{ll}1 & c \\ 0 & 1\end{array}\right)$. The local coordinates $\left\{x^{1}, x^{2}\right\}$ are such that $C_{1}=0$ and $C_{2} \neq 0$. We claim that the formula for $\widehat{\mathcal{E}}_{K}$ still makes sense. To see this, the result of parallel transport around $Y$ is $x^{1} \rightarrow x^{1}+c x^{2}$ and $x^{2} \rightarrow x^{2}$. In terms of the metric (A.24), this is the same as $\eta \rightarrow \eta, U \rightarrow U, a \rightarrow a, A \rightarrow A+c$, $G \rightarrow G-c H, H \rightarrow H$ and $K \rightarrow K$. One sees that the integrand of $(\underline{\mathrm{A} .26})$ is preserved under these changes. Hence the formula for $\widehat{\mathcal{E}}_{K}$ makes sense and (A.27) still holds.

Now suppose that $\widehat{\mathcal{E}}_{K}$ is constant in $t$. From (A.27) and (A.28), if $\operatorname{det} G$ is not constant in $t$ (in which case $G$ is locally constant in $y$ and $t$ and $B$ is flat) then $U$ and $A$ are constant in $y$ and $R$. Using the equations in [20, Proposition 4.4], one finds that the Lorentzian metric on $(0, \infty) \times Y$ is a constant times

$$
-\frac{R^{2}}{R^{2}-C K^{2}} d R^{2}+\frac{1}{R^{2}}\left(R^{2}-C K^{2}\right) e^{-2 \sigma(\theta)} d \theta^{2}
$$

where $C$ is a constant and $\sigma: Y \rightarrow \mathbb{R}$ is arbitrary. If (A.29) admits a future timelike curve along which the proper time goes to infinity then $R$ must range over an interval $\left[R_{0}, \infty\right)$. The length of the $S^{1}$-fiber is bounded as $R \rightarrow \infty$.

Remark A.30. The second equation below [20, (4.26)] should read $F:=2 U_{R} U_{\theta}+2 R^{-2} e^{4 U} A_{R} A_{\theta}$.

A.4. Monotonicity of reduced volume. In this subsection, we consider monotonic quantities when $\operatorname{dim}(B)>2$. As in Choquet-Bruhat [10] and Choquet-Bruhat-Moncrief [11, we make an appropriate conformal transformation of the Lorentzian metric on $B$ and assume that the new metric has an expanding CMC foliation. It turns out that the normalized volume of the time slice is monotonically nonincreasing.

To simplify the calculations, we start with a Lorentzian metric $\bar{g}$ of the form (A.1) and consider a conformally related metric $\bar{h}=e^{2 \bar{\phi}} \bar{g}$, where $\bar{\phi}$ pulls back from $B$. We impose the vacuum Einstein equations on $\bar{h}$. With an appropriate choice of $\bar{\phi}$, the monotonic quantity is derived from the geometry of $(B, g)$.

The papers [10] and [1] deal with the case $N=2$. The space of inner products $G$ on $\mathbb{R}^{2}$ is isomorphic to $\mathbb{R}^{+} \times H^{2}$, which gives the link between the present paper and the formalism of [10] and [11.

The monotonic quantity in this section is only defined when $\operatorname{dim}(B)>2$. If $\operatorname{dim}(B)=2$ then the formula for $\bar{\phi}$ is such that $\bar{h}$ would necessarily have a constant volume density on its $\mathbb{R}^{N}$-fibers, which need not be the case. 
To begin, we consider the effect of a conformal change on an arbitrary Lorentzian metric $\bar{g}$ on $M$. Given $\bar{\phi} \in C^{\infty}(M)$, put $\bar{h}=e^{2 \bar{\phi}} \bar{g}$. Then the Ricci curvature of $\bar{h}$ is given by

$$
\bar{R}_{a b}^{\bar{h}}=\bar{R}_{a b}^{\bar{g}}-(m-2) \bar{\phi}_{; a b}+(m-2) \bar{\phi}_{, a} \bar{\phi}_{, b}-\left(\triangle_{\bar{g}} \bar{\phi}+(m-2)|\nabla \bar{\phi}| \frac{2}{g}\right) \bar{g}_{a b} \text {. }
$$

We now assume that $\bar{g}$ is of the form (A.1). Given $\phi \in C^{\infty}(B)$, put $\bar{\phi}=\pi^{*} \phi \in C^{\infty}(M)$. Then on a fiber $\pi^{-1}(b)$,

$$
\begin{aligned}
& \bar{\phi}_{; I J}^{\bar{g}}=\frac{1}{2}\left\langle\nabla G_{I J}, \nabla \phi\right\rangle, \\
& \bar{\phi}_{; I \alpha}^{\bar{g}}=0 \\
& \bar{\phi}_{; \alpha \beta}^{\bar{g}}=\phi_{; \alpha \beta}^{g} \\
& \triangle_{\bar{g}} \bar{\phi}=\triangle_{g} \phi+\frac{1}{2}\langle\nabla \ln \operatorname{det} G, \nabla \phi\rangle .
\end{aligned}
$$

Combining (A.3), (A.31) and (A.32) gives

$$
\begin{aligned}
\bar{R}_{I J}^{\bar{h}}= & -\frac{1}{2} g^{\alpha \beta} G_{I J ; \alpha \beta}-\frac{1}{4} g^{\alpha \beta} G^{K L} G_{K L, \alpha} G_{I J, \beta}+\frac{1}{2} g^{\alpha \beta} G^{K L} G_{I K, \alpha} G_{L J, \beta}+ \\
& \frac{1}{4} g^{\alpha \gamma} g^{\beta \delta} G_{I K} G_{J L} F_{\alpha \beta}^{K} F_{\gamma \delta}^{L}-\frac{1}{2}(n+N-1)\left\langle\nabla G_{I J}, \nabla \phi\right\rangle_{g}- \\
& \left(\triangle_{g} \phi+\frac{1}{2}\langle\nabla \ln \operatorname{det} G, \nabla \phi\rangle_{g}+(n+N-1)|\nabla \phi|_{g}^{2}\right) G_{I J} \\
\bar{R}_{I \alpha}^{h}= & \frac{1}{2} g^{\gamma \delta} G_{I K} F_{\alpha \gamma ; \delta}^{K}+\frac{1}{2} g^{\gamma \delta} G_{I K ; \gamma} F_{\alpha \delta}^{K}+\frac{1}{4} g^{\gamma \delta} G_{I M} G^{K L} G_{K L ; \gamma} F_{\alpha \delta}^{M} \\
\bar{R}_{\alpha \beta}^{\bar{h}}= & R_{\alpha \beta}^{g}-\frac{1}{2} G^{I J} G_{I J ; \alpha \beta}+\frac{1}{4} G^{I J} G_{J K, \alpha} G^{K L} G_{L I, \beta}-\frac{1}{2} g^{\gamma \delta} G_{I J} F_{\alpha \gamma}^{I} F_{\beta \delta}^{J}- \\
& (n+N-1) \phi_{; \alpha \beta}+(n+N-1) \phi_{, \alpha} \phi_{, \beta}- \\
& \left(\triangle_{g} \phi+\frac{1}{2}\langle\nabla \ln \operatorname{det} G, \nabla \phi\rangle_{g}+(n+N-1)|\nabla \phi|_{g}^{2}\right) g_{\alpha \beta} .
\end{aligned}
$$

We now set

$$
\phi=-\frac{1}{2(n+N-1)} \ln \operatorname{det} G,
$$

so that

$$
\frac{1}{2}\langle\nabla \ln \operatorname{det} G, \nabla \phi\rangle_{g}+(n+N-1)|\nabla \phi|_{g}^{2}=0 .
$$

We set the left-hand side of (A.33) to be zero. Multiplying the first equation of (A.33) by $G^{I J}$, summing over $I$ and $J$, and using the equation

$$
\triangle_{g} \ln \operatorname{det} G=g^{\alpha \beta} G^{I J} G_{I J ; \alpha \beta}-g^{\alpha \beta} G^{I J} G_{J K, \alpha} G^{K L} G_{L K, \beta},
$$


gives

$$
\begin{aligned}
0= & -\frac{1}{2} \triangle_{g} \ln \operatorname{det} G-\frac{1}{4}|\nabla \ln \operatorname{det} G|_{g}^{2}+\frac{1}{4} g^{\alpha \gamma} g^{\beta \delta} G_{I J} F_{\alpha \beta}^{I} F_{\gamma \delta}^{J}- \\
& \frac{1}{2}(n+N-1)\langle\nabla \ln \operatorname{det} G, \nabla \phi\rangle_{g}-N \triangle_{g} \phi \\
= & \frac{1-n}{2(n+N-1)} \triangle_{g} \ln \operatorname{det} G+\frac{1}{4} g^{\alpha \gamma} g^{\beta \delta} G_{I J} F_{\alpha \beta}^{I} F_{\gamma \delta}^{J} .
\end{aligned}
$$

Using the equation

$$
(\ln \operatorname{det} G)_{; \alpha \beta}=G^{I J} G_{I J ; \alpha \beta}-G^{I J} G_{J K, \alpha} G^{K L} G_{L I, \beta} \text {, }
$$

the last equation of (A.33) becomes

$$
\begin{aligned}
0= & R_{\alpha \beta}^{g}-\frac{1}{2}(\ln \operatorname{det} G)_{; \alpha \beta}-\frac{1}{4} G^{I J} G_{J K, \alpha} G^{K L} G_{L I, \beta}-\frac{1}{2} g^{\gamma \delta} G_{I J} F_{\alpha \gamma}^{I} F_{\beta \delta}^{J}- \\
& (n+N-1) \phi_{; \alpha \beta}+(n+N-1) \phi_{, \alpha} \phi_{, \beta}-\left(\triangle_{g} \phi\right) g_{\alpha \beta} \\
= & R_{\alpha \beta}^{g}-\frac{1}{4} G^{I J} G_{J K ; \alpha} G^{K L} G_{L I ; \beta}-\frac{1}{2} g^{\gamma \delta} G_{I J} F_{\alpha \gamma}^{I} F_{\beta \delta}^{J}+ \\
& \frac{1}{4(n+N-1)}(\ln \operatorname{det} G)_{, \alpha}(\ln \operatorname{det} G)_{, \beta}+\frac{1}{2(n+N-1)}\left(\triangle_{g} \ln \operatorname{det} G\right) g_{\alpha \beta} .
\end{aligned}
$$

Using (A.37), if $n>1$ then

$$
\begin{aligned}
R_{\alpha \beta}^{g}= & \frac{1}{4} G^{I J} G_{J K, \alpha} G^{K L} G_{L I, \beta}+\frac{1}{2} g^{\gamma \delta} G_{I J} F_{\alpha \gamma}^{I} F_{\beta \delta}^{J}- \\
& \frac{1}{4(n+N-1)}(\ln \operatorname{det} G)_{, \alpha}(\ln \operatorname{det} G)_{, \beta}-\frac{1}{4(n-1)} g^{\mu \nu} g^{\gamma \delta} G_{I J} F_{\mu \gamma}^{I} F_{\nu \delta}^{J} g_{\alpha \beta} .
\end{aligned}
$$

In terms of the decomposition (A.9), let $K_{i j}$ be the second fundamental form of the spatial hypersurfaces. By performing a gauge transformation, we can assume that $A_{0}=0$. 
Put

$$
\begin{aligned}
H & =h^{i j} K_{i j} \\
K_{i j}^{0} & =K_{i j}-\frac{1}{n} H h_{i j}, \\
|K|^{2} & =K^{i j} K_{i j} \\
\left|K^{0}\right|^{2} & =K^{0, i j} K_{i j}^{0}=|K|^{2}-\frac{1}{n} H^{2} . \\
\left|\frac{\partial G}{\partial t}\right|^{2} & =\operatorname{Tr}\left(\left(G^{-1} G_{, 0}\right)^{2}\right)=G^{I J} G_{J K, 0} G^{K L} G_{L I, 0}, \\
|\nabla G|_{G, h}^{2} & =h^{i j} \operatorname{Tr}\left(G^{-1} G_{, i} G^{-1} G_{, j}\right)=h^{i j} G^{I J} G_{J K, i} G^{K L} G_{L I, j}, \\
S_{\alpha} & =G^{-\frac{1}{2}} G_{, \alpha} G^{-\frac{1}{2}}-\frac{1}{N}\left(\ln \operatorname{det} G_{, \alpha} I_{N},\right. \\
\left|S_{0}\right|^{2} & =\operatorname{Tr}\left(S_{0}^{2}\right)=\left|\frac{\partial G}{\partial t}\right|^{2}-\frac{1}{N}\left(\frac{\partial \ln \operatorname{det} G}{\partial t}\right)^{2}, \\
|\vec{S}|^{2} & =h^{i j} \operatorname{Tr}\left(S_{i} S_{j}\right)=h^{i j} \operatorname{Tr}\left(G^{-1} G_{, i} G^{-1} G_{, j}\right)-\frac{1}{N}|\nabla \ln \operatorname{det} G|^{2} .
\end{aligned}
$$

Then from the Gauss-Codazzi equation,

$$
R_{00}^{g}-\frac{1}{2} R^{g} g_{00}=\frac{L^{2}}{2}\left(R^{h}-|K|^{2}+H^{2}\right)=\frac{L^{2}}{2}\left(R^{h}-\left|K^{0}\right|^{2}+\left(1-\frac{1}{n}\right) H^{2}\right) .
$$

From (A.40),

$$
\begin{aligned}
R_{00}^{g}-\frac{1}{2} R^{g} g_{00}= & \frac{1}{8}\left|\frac{\partial G}{\partial t}\right|^{2}+\frac{1}{8} L^{2}|\nabla G|_{G, h}^{2}- \\
& \frac{1}{8(n+N-1)}\left(\frac{\partial \ln \operatorname{det} G}{\partial t}\right)^{2}-\frac{1}{8(n+N-1)} L^{2}|\nabla \ln \operatorname{det} G|_{h}^{2}+ \\
& \frac{1}{4} h^{i j} G_{I J} F_{0 i}^{I} F_{0 j}^{J}+\frac{1}{8} L^{2} h^{i k} h^{j l} G_{I J} F_{i j}^{I} F_{k l}^{J} .
\end{aligned}
$$

Hence we obtain the constraint equation

$$
\begin{aligned}
& L^{2}\left(R^{h}-\left|K^{0}\right|^{2}+\left(1-\frac{1}{n}\right) H^{2}\right)= \\
& \frac{1}{4}\left|\frac{\partial G}{\partial t}\right|^{2}+\frac{1}{4} L^{2}|\nabla G|_{G, h}^{2}- \\
& \frac{1}{4(n+N-1)}\left(\frac{\partial \ln \operatorname{det} G}{\partial t}\right)^{2}-\frac{1}{4(n+N-1)} L^{2}|\nabla \ln \operatorname{det} G|_{h}^{2}+ \\
& \frac{1}{2} h^{i j} G_{I J} F_{0 i}^{I} F_{0 j}^{J}+\frac{1}{4} L^{2} h^{i k} h^{j l} G_{I J} F_{i j}^{I} F_{k l}^{J}
\end{aligned}
$$


or, equivalently,

$$
\begin{aligned}
& L^{2}\left(R^{h}-\left|K^{0}\right|^{2}+\left(1-\frac{1}{n}\right) H^{2}\right)= \\
& \frac{1}{4}\left|S_{0}\right|^{2}+\frac{1}{4} L^{2}|\vec{S}|^{2}+ \\
& \frac{n-1}{4 N(n+N-1)}\left(\frac{\partial \ln \operatorname{det} G}{\partial t}\right)^{2}+\frac{n-1}{4 N(n+N-1)} L^{2}|\nabla \ln \operatorname{det} G|_{h}^{2}+ \\
& \frac{1}{2} h^{i j} G_{I J} F_{0 i}^{I} F_{0 j}^{J}+\frac{1}{4} L^{2} h^{i k} h^{j l} G_{I J} F_{i j}^{I} F_{k l}^{J} .
\end{aligned}
$$

From the spacetime splitting,

$$
\frac{\partial h_{i j}}{\partial t}=-2 L K_{i j}
$$

and

$$
\frac{\partial K_{i j}}{\partial t}=L H K_{i j}-2 L h^{k l} K_{i k} K_{l j}-L_{; i j}+L R_{i j}^{h}-L R_{i j}^{g}
$$

where the covariant derivatives are now with respect to $h$. Then using (A.40), (A.41) and (A.45),

$$
\begin{aligned}
\frac{\partial H}{\partial t}= & L H^{2}-\triangle_{h} L+L R^{h}-L h^{i j} R_{i j}^{g} \\
= & L H^{2}-\triangle_{h} L+L R^{h}-\frac{1}{4} L|\nabla G|_{G, h}^{2}+\frac{1}{4(n+N-1)} L|\nabla \ln \operatorname{det} G|_{h}^{2}- \\
& \frac{1}{2(n-1)} L^{-1} h^{i j} G_{I J} F_{0 i}^{I} F_{0 j}^{J}-\frac{n-2}{4(n-1)} L h^{i j} h^{k l} G_{I J} F_{i k}^{I} F_{j l}^{J} \\
= & -\triangle_{h} L+L\left|K^{0}\right|^{2}+\frac{1}{n} L H^{2}+ \\
& \frac{1}{4} L^{-1}\left|S_{0}\right|^{2}+\frac{n-1}{4 N(n+N-1)} L^{-1}\left(\frac{\partial \ln \operatorname{det} G}{\partial t}\right)^{2}+ \\
& \frac{n-2}{2(n-1)} L^{-1} h^{i j} G_{I J} F_{0 i}^{I} F_{0 j}^{J}+\frac{1}{4(n-1)} L h^{i j} h^{k l} G_{I J} F_{i k}^{I} F_{j l}^{J} .
\end{aligned}
$$

Now suppose that $H$ is spatially constant but time-dependent. The maximum principle, when applied to (A.48), gives

$$
L \leq \frac{n}{H^{2}} \frac{\partial H}{\partial t}
$$

We have the pointwise identity

$$
\frac{\partial}{\partial t} \operatorname{dvol}(Y, h)=\frac{1}{2} h^{i j} \frac{\partial h_{i j}}{\partial t} \operatorname{dvol}_{h}=-L H \operatorname{dvol}_{h},
$$


SO

$$
\frac{\partial}{\partial t}\left((-H)^{n} \operatorname{dvol}(Y, h)\right)=(-H)^{n+1}\left(L-\frac{n}{H^{2}} \frac{\partial H}{\partial t}\right) \operatorname{dvol}(Y, h) .
$$

Assuming that $H$ is negative, it follows from (A.49) and (A.51) that $(-H)^{n} \operatorname{dvol}(Y, h(t))$ is pointwise monotonically nonincreasing in $t$, and hence $(-H)^{n} \operatorname{vol}(Y, h(t))$ is monotonically nonincreasing in $t$. Applying (A.48) to (A.51) gives

$$
\begin{aligned}
& \frac{d}{d t}\left((-H)^{n} \operatorname{vol}(Y, h)\right)=-n(-H)^{n-1} \int_{Y}\left[L\left|K^{0}\right|^{2}+\frac{1}{4} L^{-1}\left|S_{0}\right|^{2}+\right. \\
& \frac{n-1}{4 N(n+N-1)} L^{-1}\left(\frac{\partial \ln \operatorname{det} G}{\partial t}\right)^{2}+\frac{n-2}{2(n-1)} L^{-1} h^{i j} G_{I J} F_{0 i}^{I} F_{0 j}^{J}+ \\
& \left.\frac{1}{4(n-1)} L h^{i j} h^{k l} G_{I J} F_{i k}^{I} F_{j l}^{J}\right] \operatorname{dvol}(Y, h) .
\end{aligned}
$$

We note in passing that (A.45) gives an energy-type interpretation for the normalized volume, as

$$
\begin{aligned}
& (-H)^{n} \operatorname{vol}(Y, h)= \\
& \frac{n}{n-1}(-H)^{n-2} \int_{Y}\left[-R^{h}+\left|K^{0}\right|^{2}+\frac{1}{4} L^{-2}\left|S_{0}\right|^{2}+\frac{1}{4}|\vec{S}|^{2}+\right. \\
& \frac{n-1}{4(n+N-1)} L^{-2}\left(\frac{\partial \ln \operatorname{det} G}{\partial t}\right)^{2}+\frac{n-1}{4(n+N-1)}|\nabla \ln \operatorname{det} G|_{h}^{2}+ \\
& \left.\frac{1}{2} L^{-2} h^{i j} G_{I J} F_{0 i}^{I} F_{0 j}^{J}+\frac{1}{4} h^{i k} h^{j l} G_{I J} F_{i j}^{I} F_{k l}^{J}\right] \operatorname{dvol}_{Y} .
\end{aligned}
$$

If $(-H)^{n} \operatorname{vol}(Y, h)$ is constant in $t$ and $n>2$ then from (A.52), we must have

$$
0=K^{0}=S_{0}=\frac{\partial \ln \operatorname{det} G}{\partial t}=F_{i j}^{I}=F_{0 i}^{I} .
$$

Then $K_{i j}=\frac{1}{n} H h_{i j}$, the connection $A_{i}^{I}$ is spatially flat and time-independent, and $G$ is time-independent. Equation (A.48) now has the unique solution

$$
L=n H^{-2} \frac{d H}{d t} \text {. }
$$

From (A.46),

$$
\frac{\partial h_{i j}}{\partial t}=-2 H^{-1} \frac{d H}{d t} h_{i j},
$$

so

$$
h_{i j}(t)=H^{-2}(t) H^{2}(1) h_{i j}(1) .
$$

From (A.37), we have $\triangle_{h} \ln \operatorname{det} G=0$, so $\ln \operatorname{det} G$ is constant. Then from the first equation in (A.33), $G$ satisfies

$$
0=h^{i j} G_{I J ; i j}-h^{i j} G^{K L} G_{I K, i} G_{L J, j},
$$


where the covariant derivatives are now with respect to $h$. Equations (A.40) and (A.47) now give

$$
\begin{aligned}
R_{i j}^{h} & =-\frac{n-1}{n^{2}} H^{2} h_{i j}+R_{i j}^{g} \\
& =-\frac{n-1}{n^{2}} H^{2} h_{i j}+\frac{1}{4} G^{I J} G_{J K, i} G^{K L} G_{L I, j} .
\end{aligned}
$$

Conversely, given a static solution $\left(h_{i j}, G_{I J}\right)$ to the pair

$$
\begin{aligned}
0 & =h^{i j} G_{I J ; i j}-h^{i j} G^{K L} G_{I K, i} G_{L J, j}, \\
R_{i j}^{h} & =-(n-1) h_{i j}+\frac{1}{4} G^{I J} G_{J K, i} G^{K L} G_{L I, j},
\end{aligned}
$$

and an increasing positive function $\sigma(t)$, we get a solution

$$
\begin{aligned}
L(t) & =\frac{d \sigma}{d t}, \\
h_{i j}(t) & =\sigma^{2}(t) h_{i j}, \\
K_{i j}(t) & =-\sigma(t) h_{i j} \\
G_{I J}(t) & =G_{I J}
\end{aligned}
$$

with $H(t)=-\frac{n}{\sigma(t)}$. Solutions to (A.60) are discussed in [22, Proposition 4.80].

A.4.1. The case $n=2$. If $n=2$ and $(-H)^{2} \operatorname{vol}(Y, h)$ is constant in $t$ then from (A.52),

$$
0=K^{0}=S_{0}=\frac{\partial \ln \operatorname{det} G}{\partial t}=F_{i j}^{I}
$$

and so

$$
L=n H^{-2} \frac{d H}{d t}
$$

Equation (A.37) becomes

$$
\frac{1}{N+1} \triangle_{h} \ln \operatorname{det} G=-L^{-2} h^{i j} G_{I J} F_{0 i}^{I} F_{0 j}^{J} .
$$

Integrating over $Y$ gives $F_{0 i}^{I}=0$. The discussion in (A.56)-(A.61) is now valid.

\section{REFERENCES}

[1] M. Anderson, "Scalar curvature, metric degeneration and the static vacuum Einstein equations on 3-manifolds I", Geom. Funct. Anal. 9, p. 855-967 (1999)

[2] M. Anderson, "On long-time evolution in general relativity and geometrization of 3-manifolds", Comm. Math. Phys. 222, p. 533-567 (2001)

[3] L. Andersson and V. Moncrief, "Future complete vacuum spacetimes", in The Einstein equations and the large scale behavior of gravitational fields, eds. P. Chruściel and H. Friedrich, Birkhäuser, Basel, p. 299-330 (2004)

[4] R. Bamler, "Long-time behavior of 3 dimensional Ricci flow - Introduction", Geometry and Topology 22 , p. 757-774 (2018) 
[5] B. Berger, P. Chruściel, J. Isenberg and V. Moncrief, "Global foliations of vacuum spacetimes with $T^{2}$ isometry", Annals of Physics 260, p. 117-148 (1997)

[6] J. Cheeger and M. Gromov, "Collapsing Riemannian manifolds while keeping the curvature bounded I", J. Diff. Geom. 23, p. 309-346 (1986)

[7] J. Cheeger and M. Gromov, "Collapsing Riemannian manifolds while keeping the curvature bounded II", J. Diff. Geom. 32, p. 269-298 (1990)

[8] J. Cheeger, M. Gromov and M. Taylor, "Finite propagation speed, kernel estimates for functions of the Laplace operator, and the geometry of complete Riemannian manifolds", J. Diff. Geom. 17, p. 15-53 (1982)

[9] B.-L. Chen and P. LeFloch, "Local foliations and optimal regularity of Einstein spacetimes", J. Geom. Phys. 29, p. 913-941 (2009)

[10] Y. Choquet-Bruhat, "Future complete $U(1)$ symmetric Einsteinian spacetimes, the unpolarized case", in The Einstein equations and large scale behavior of gravitational fields, eds. P. Chruściel and H. Friedrich, Birkhäuser, Basel, p. 251-298 (2004)

[11] Y. Choquet-Bruhat and V. Moncrief, "Future global in time Einstein spacetimes with $U(1)$ isometry group", Annales Henri Poincaré 2, p. 1007-1064 (2001)

[12] B. Chow, P. Lu, and L. Ni, Hamilton's Ricci flow, Grad. Stud. Math. 77, Amer. Math. Soc., Providence, RI (2006)

[13] P. Chruściel, J. Isenberg and D. Pollack, "Initial data engineering", Comm. Math. Phys. 257, p. 29-42 (2005)

[14] D. Eardley, J. Isenberg, J. Marsden and V. Moncrief, "Homothetic and conformal symmetries of solutions to Einstein's equations", Comm. Math. Phys. 106, p. 137-158 (1986)

[15] G. Ellis and J. Wainwright, eds, Dynamical systems in cosmology, Cambridge University Press, Cambridge (1997)

[16] A. Fischer and V. Moncrief, "Hamiltonian reduction and perturbations of continuously self-similar $(n+1)$-dimensional Einstein vacuum spacetimes", Class. Quantum Grav. 19, p. 5557-5589 (2002)

[17] M. Gromov, "Groups of polynomial growth and expanding maps", Publ. Math. IHES 53, p. 53-73 (1981)

[18] R. Hamilton, "A compactness property for solutions of the Ricci flow", R. Hamilton, Amer. J. Math. 117 , p. $545-572(1995)$

[19] C. Hilaire, "Ricci flow on Riemannian groupoids", preprint, http://arxiv.org/abs/1411.6058 (2015)

[20] P. LeFloch and J. Smulevici, "Weakly regular $T^{2}$-symmetric spacetimes. The global geometry of future Cauchy developments", J. Eur. Math. Soc. 17,p. 1229-1292 (2015)

[21] J. Lott, "On the long-time behavior of type-III Ricci flow solutions", Math. Ann. 339, p. 627-666 (2007)

[22] J. Lott, "Dimensional reduction and the long-time behavior of Ricci flow", Comm. Math. Helv. 85, p. $485-534$ (2010)

[23] M. Reiris, "The ground state and the long-time evolution in the CMC Einstein flow", Ann. Henri Poincaré 10, p. 1559-1604 (2010)

[24] H. Ringstrom, "Future asymptotics expansions of Bianchi VIII vacuum metrics", Class. Quantum Grav. 20, p. 1943-1989 (2003)

[25] H. Ringstrom, "On curvature decay in expanding cosmological models", Comm. Math. Phys. 264, p. 613-630 (2006)

[26] H. Ringstrom, "Instability of spatially homogeneous solutions in the class of $T^{2}$-symmetric solutions to Einstein's vacuum equations", Comm. Math. Phys. 334, p. 1299-1375 (2015)

[27] P. Scott, "The geometries of 3-manifolds", Bull. London Math. Soc. 15, p. 401-487 (1983)

[28] A. Spiro, "A remark on locally homogeneous Riemannian spaces", Results Math. 24, p. 318-325 (1993)

[29] S.-T. Yau, "Harmonic functions on complete Riemannian manifolds", Comm. Pure and Appl. Math. 28 , p. $201-228(1975)$ 
Department of Mathematics, University of California, Berkeley, Berkeley, CA 947203840 , USA

E-mail address: lott@berkeley.edu 